Supporting Information for

\title{
Multielectron Redox Chemistry of Iron Porphyrinogens
}

\author{
Julien Bachmann and Daniel G. Nocera * \\ Department of Chemistry, 6-335, Massachusetts Institute of Technology, 77 Massachusetts \\ Avenue, Cambridge, MA 02139-4307
}

Index

Page

X-ray crystal structure of $\left[(\mathrm{THF})_{3} \mathrm{Fe}^{\mathrm{II}}(\mu-\mathrm{Cl})_{3} \mathrm{Fe}^{\mathrm{II}}(\mathrm{THF})_{3}\right]\left[(\mathrm{THF})_{4} \mathrm{Fe}^{\mathrm{II}}(\mu-\right.$ $\left.\mathrm{Cl})_{2} \mathrm{Fe}^{\mathrm{II}}(\mathrm{THF})_{4}\right]_{1 / 2}\left[\mathrm{LFe}^{\mathrm{III}}\right]_{2} \cdot 2 \mathrm{PhCH}_{3}$ and selected metrics

Cyclic voltammetry of the $\left[\mathrm{LFe}^{\mathrm{III}}\right]^{-} /\left[\mathrm{LFe}^{\mathrm{II}}\right]^{2-}$ redox couple

X-ray crystal structure data for $\left[\mathrm{Na}(\text { diglyme })_{2}\right]\left[\mathrm{LFe}^{\mathrm{III}}\right]$

X-ray crystal structure data for $\left[(\mathrm{THF})_{3} \mathrm{Fe}^{\mathrm{II}}(\mu-\mathrm{Cl})_{3} \mathrm{Fe}^{\mathrm{II}}(\mathrm{THF})_{3}\right]$

$\left[(\mathrm{THF})_{4} \mathrm{Fe}^{\mathrm{II}}(\mu-\mathrm{Cl})_{2} \mathrm{Fe}^{\mathrm{II}}(\mathrm{THF})_{4}\right]_{1 / 2}\left[\mathrm{LFe}^{\mathrm{III}}\right]_{2} \cdot 2 \mathrm{PhCH}_{3}$

S16-S37

X-ray crystal structure data for $[(\mathrm{THF}) \mathrm{Na}(\mathrm{Opy})]_{2}\left[\mathrm{LFe}^{\mathrm{II}}\right]$

S38-S50

X-ray crystal structure data for $\left[\mathrm{L}^{\Delta \Delta} \mathrm{Fe}^{\mathrm{II}}\left(\mathrm{NCCH}_{3}\right)\right]$

$\left[\left(\mathrm{C}_{2} \mathrm{~B}_{9} \mathrm{H}_{11}\right)_{2} \mathrm{Co}\right]_{2} \cdot 2 \mathrm{CH}_{3} \mathrm{CN} \cdot 2 o-\mathrm{C}_{6} \mathrm{H}_{4} \mathrm{C}_{12}$

Calculated energies of the frontier and near-frontier orbitals

of $\left[\mathrm{LFe}^{\mathrm{II}}\right]^{2-}$ and Mulliken population analysis

Calculated energies of the frontier and near-frontier orbitals of $\left[\mathrm{LFe}^{\mathrm{III}}\right]^{-}$and Mulliken population analysis

Calculated energies of the frontier and near-frontier orbitals of $\left[\mathrm{L}^{\Delta \Delta} \mathrm{Fe}^{\mathrm{II}}\left(\mathrm{NCCH}_{3}\right)\right]^{2+}$ and Mulliken population analysis

Calculated single-point energies of the different spin states of the iron porphyrinogens 


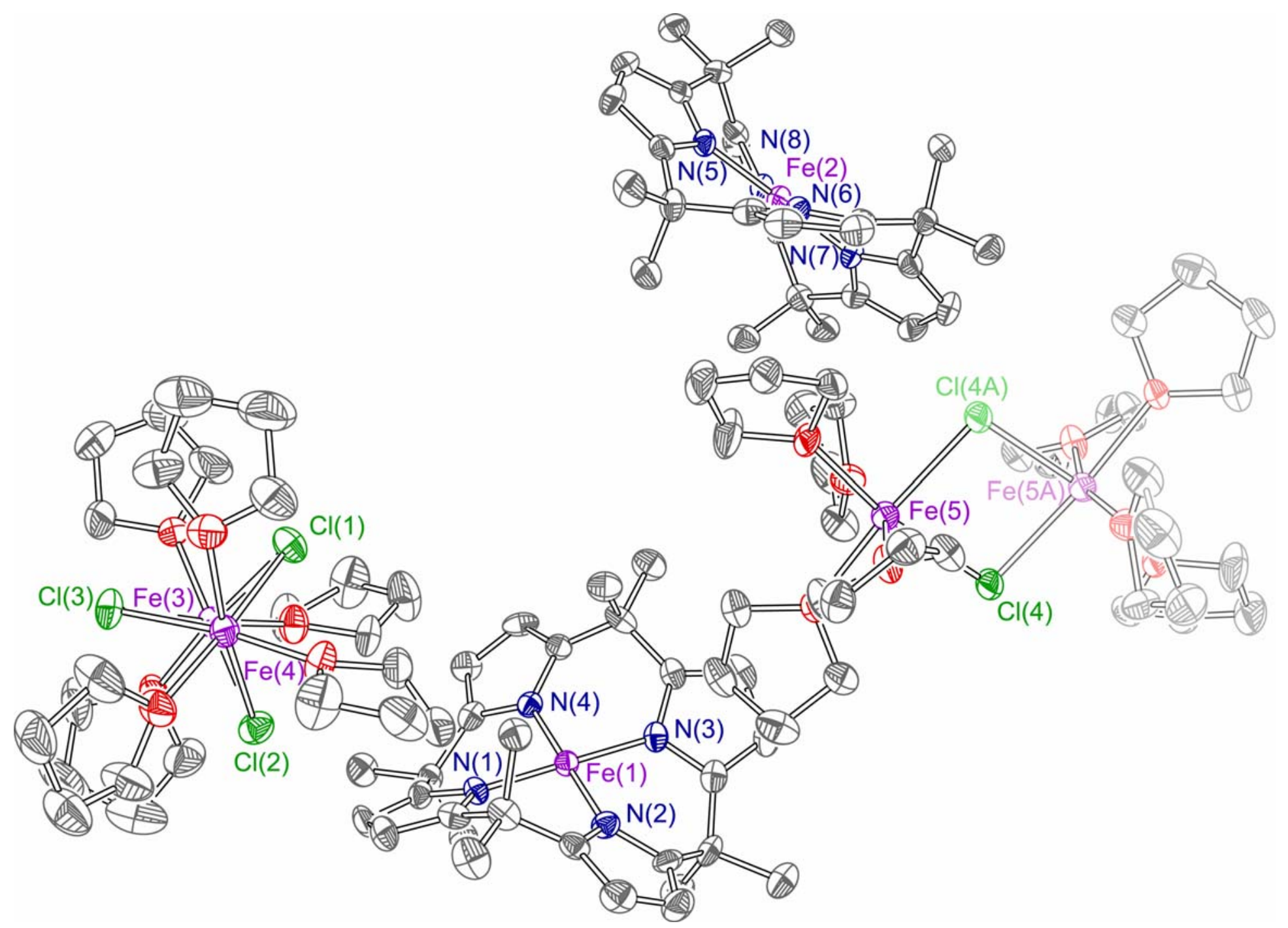

Figure S1. Solid-state structure of $\left[(\mathrm{THF})_{3} \mathrm{Fe}^{\mathrm{II}}(\mu-\mathrm{Cl})_{3} \mathrm{Fe}^{\mathrm{II}}(\mathrm{THF})_{3}\right]\left[(\mathrm{THF})_{4} \mathrm{Fe}^{\mathrm{II}}(\mu-\mathrm{Cl})_{2} \mathrm{Fe}^{\mathrm{II}}(\mathrm{THF})_{4}\right]_{1 / 2}\left[\mathrm{LFe}^{\mathrm{III}}\right]_{2}$ in a crystal grown from $\mathrm{THF} /$ toluene. Thermal ellipsoids are drawn at the 50\% probability level (color coding: purple, Fe; blue, N; red, O; green, Cl; gray, C); $\mathrm{H}$ atoms as well as two solvent toluene molecules are omitted for clarity. The contents of the asymmetric unit are shown in normal colors, and one half of the $\mathrm{Fe}_{2} \mathrm{Cl}_{2}{ }^{2+}$ ion is added in faded colors for completeness. 
Table S1. Assignment of the $\mathrm{Fe}$ formal oxidation states in $\left[(\mathrm{THF})_{3} \mathrm{Fe}^{\mathrm{II}}(\mu-\mathrm{Cl})_{3} \mathrm{Fe}^{\mathrm{II}}(\mathrm{THF})_{3}\right]\left[(\mathrm{THF})_{4} \mathrm{Fe}^{\mathrm{II}}(\mu-\mathrm{Cl})_{2} \mathrm{Fe}^{\mathrm{II}}(\mathrm{THF})_{4}\right]_{1 / 2}\left[\mathrm{LFe}^{\mathrm{III}}\right]_{2}$ by comparison of the metric parameters of the $\left[(\mathrm{THF})_{3} \mathrm{Fe}(\mu-\mathrm{Cl})_{3} \mathrm{Fe}(\mathrm{THF})_{3}\right]^{+}$(here referred to as $\left[\mathrm{Fe}_{2}\right]^{+}$) and $[\mathrm{LFe}]^{-}$entities with other structures featuring no oxidation state ambiguity.

\begin{tabular}{|c|c|c|c|}
\hline & & structure in Figure $\mathrm{S} 1$ & as compared to \\
\hline \multirow{2}{*}[\mathrm{Fe}_{2}]{$^{+}$cation } & $\mathrm{d}(\mathrm{Fe}-\mathrm{Cl})_{\mathrm{avg}} / \AA$ & $2.48( \pm 0.05)$ & $2.49( \pm 0.01)^{a}$ \\
\hline & $\angle(\mathrm{Cl}-\mathrm{Fe}-\mathrm{Cl})_{\mathrm{avg}} /{ }^{\mathrm{o}}$ & $86.8( \pm 1.9)$ & $85.8( \pm 0.6)^{a}$ \\
\hline$[\mathrm{LFe}]^{-}$anion & $\mathrm{d}(\mathrm{Fe}-\mathrm{N})_{\mathrm{avg}} / \AA$ & $1.903( \pm 0.010)$ & $1.899( \pm 0.005)^{b}$ \\
\hline
\end{tabular}




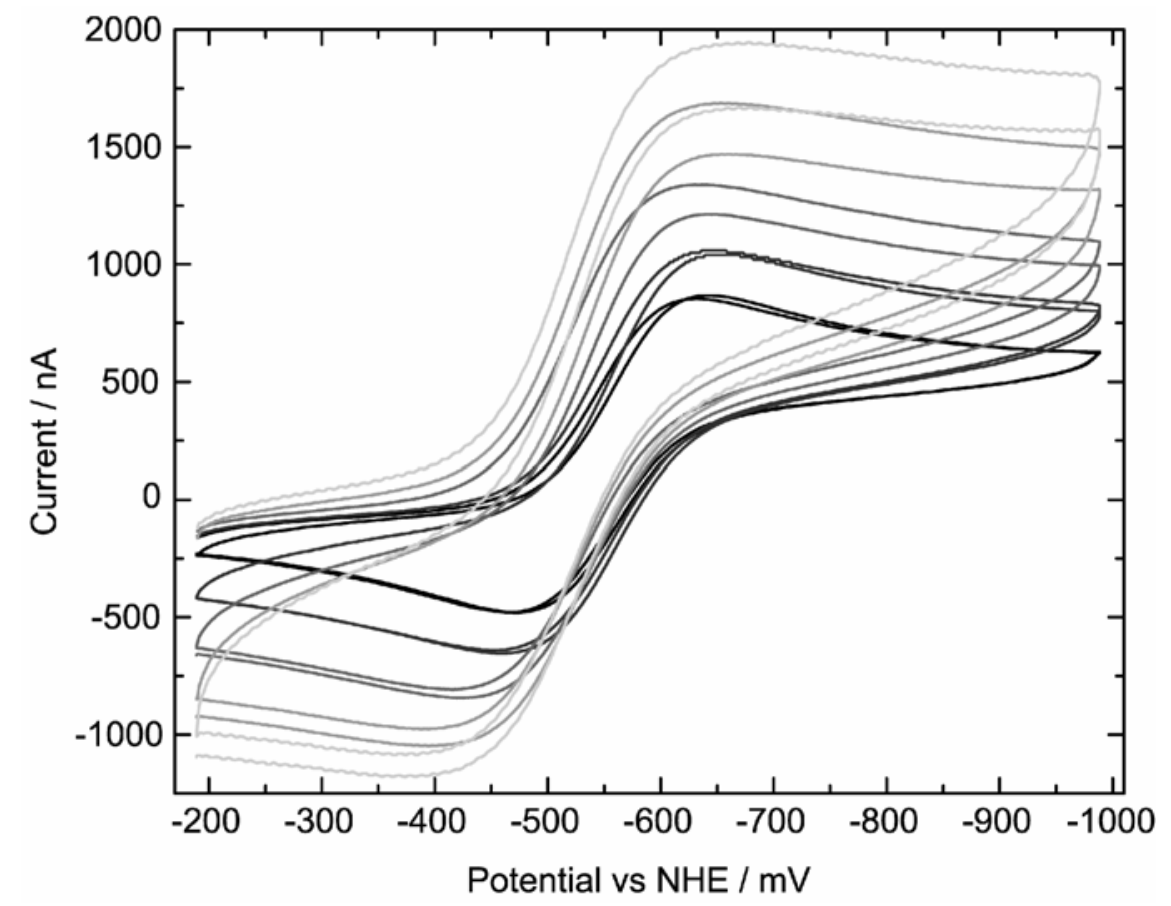

Figure S2. Reversible $\left[\mathrm{LFe}^{\mathrm{III}}\right]^{-} /\left[\mathrm{LFe}^{\mathrm{II}}\right]^{2-}$ wave in the cyclic voltammetry of $\mathrm{CH}_{3} \mathrm{CN}$ solutions containing $1 \mathrm{mM}\left(\mathrm{Bu}_{4} \mathrm{~N}\right)\left[\mathrm{LFe}^{\mathrm{III}}\right]$ in $0.04 \mathrm{M}\left(\mathrm{Bu}_{4} \mathrm{~N}\right)\left(\mathrm{BPh}_{4}\right)$; scan rate $/ \mathrm{mV} \mathrm{s}^{-1}$ (from black to light gray, two full scans each): 50, 100, 200, 400, 600 . 


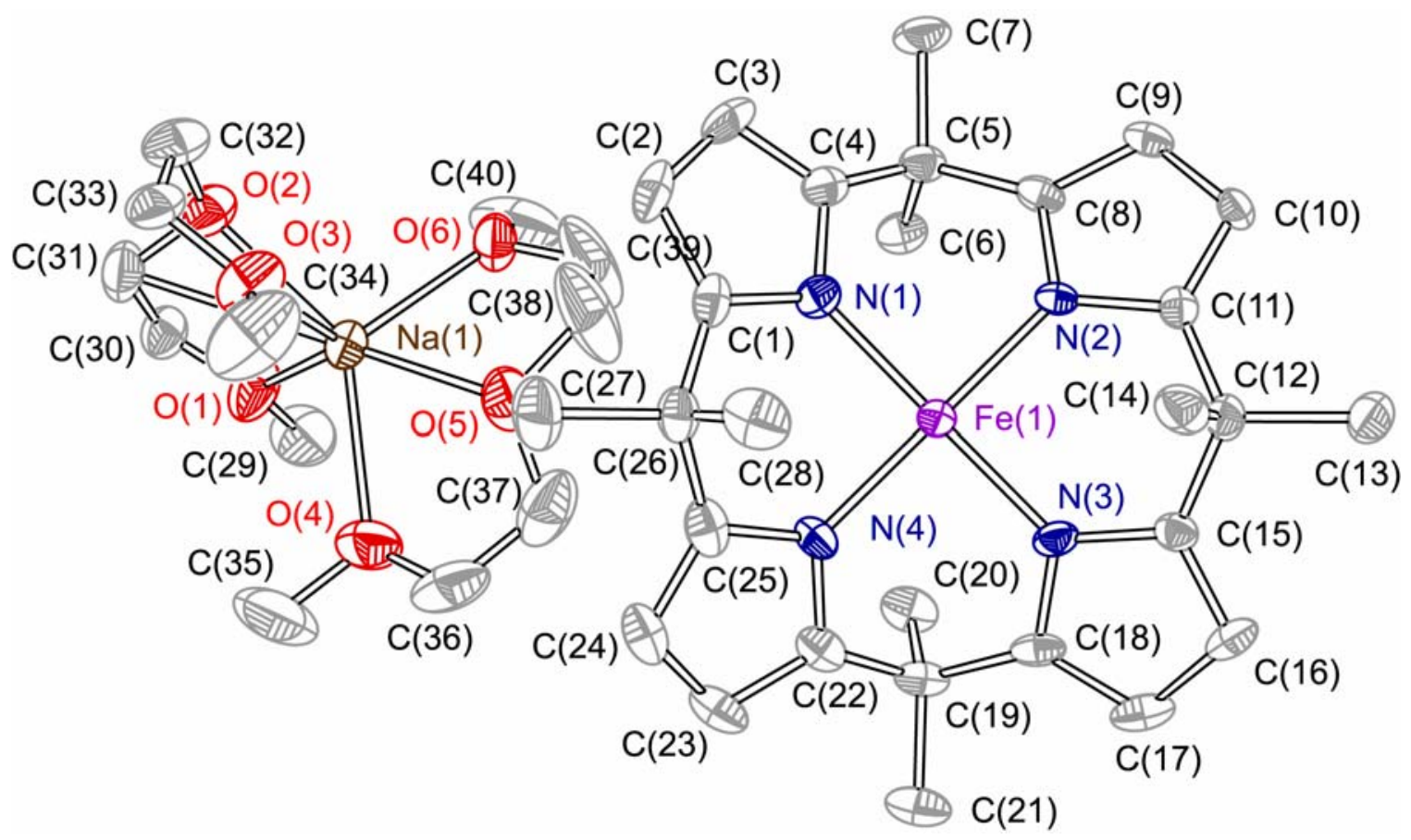

Figure S3. Atom labeling scheme for $\left[\mathrm{Na}(\text { diglyme })_{2}\right]\left[\mathrm{LFe}^{\mathrm{III}}\right]$. 
Table S2. Crystal data and structure refinement for $\left[\mathrm{Na}(\text { diglyme })_{2}\right]\left[\mathrm{LFe}^{\mathrm{III}}\right]$.

\begin{tabular}{|c|c|}
\hline Empirical formula & $\mathrm{C}_{40} \mathrm{H}_{60} \mathrm{FeN}_{4} \mathrm{O}_{6}$ \\
\hline Formula weight & 771.76 \\
\hline$T(\mathrm{~K})$ & $183(2)$ \\
\hline$\lambda(\AA)$ & 0.71073 \\
\hline Crystal system & Orthorhombic \\
\hline Space group & $P_{\text {bca }}$ \\
\hline$a(\AA)$ & $15.5367(12)$ \\
\hline$b(\AA)$ & $21.1012(16)$ \\
\hline$c(\AA)$ & $25.2473(19)$ \\
\hline$\alpha\left(^{\circ}\right)$ & 90 \\
\hline$\beta\left(^{\circ}\right)$ & 90 \\
\hline$\gamma\left({ }^{\circ}\right)$ & 90 \\
\hline$V / \AA^{3}$ & $8277.2(11)$ \\
\hline$Z$ & 8 \\
\hline$\rho_{\text {calcd }}\left(\mathrm{g} / \mathrm{cm}^{3}\right)$ & 1.239 \\
\hline Absorption coefficient $\left(\mathrm{mm}^{-1}\right)$ & 0.423 \\
\hline$F(000)$ & 3304 \\
\hline Crystal size $\left(\mathrm{mm}^{3}\right)$ & $0.45 \times 0.45 \times 0.45$ \\
\hline$\theta$ range for data collection & 2.29 to $23.28^{\circ}$ \\
\hline Index ranges & $-16 \leq h \leq 17,-20 \leq k \leq 23,-27 \leq \ell \leq 28$ \\
\hline No. of coll. reflns & 31601 \\
\hline No. of ind. reflns $\left(\mathrm{R}_{\text {int }}\right)$ & $5951(0.0582)$ \\
\hline Completeness to $\theta=23.30^{\circ}$ & $99.8 \%$ \\
\hline Absorption correction & None \\
\hline Refinement method & Full-matrix least-squares on $F^{2}$ \\
\hline Data / restraints / parameters & $5951 / 0 / 482$ \\
\hline $\mathrm{GOF}^{a}$ on $F^{2}$ & 1.479 \\
\hline$R 1,{ }^{b} \mathrm{w} R 2^{c}(\mathrm{I}>2 \sigma)$ & $0.0962,0.1867$ \\
\hline$R 1,{ }^{b} \mathrm{w} R 2^{c}$ (all data) & $0.0962,0.1867$ \\
\hline Extinction coefficient & $0.00334(18)$ \\
\hline Largest diff. peak and hole (e. $\left.\AA^{-3}\right)$ & 1.003 and -0.605 \\
\hline
\end{tabular}


Table S3. Atomic coordinates $\left(\times 10^{4}\right)$ and equivalent isotropic displacement parameters $\left(\AA^{2} \times 10^{3}\right)$ for $\left[\mathrm{Na}(\text { diglyme })_{2}\right]\left[\mathrm{LFe}^{\mathrm{III}}\right]$.

\begin{tabular}{|c|c|c|c|c|}
\hline Atom & $\mathrm{x}$ & $\mathrm{y}$ & z & $\mathrm{U}_{\mathrm{eq}}$ \\
\hline $\mathrm{Fe}(1)$ & $8458(1)$ & $933(1)$ & $6167(1)$ & $21(1)$ \\
\hline $\mathrm{Na}(1)$ & $9654(2)$ & $2350(1)$ & $3408(1)$ & $37(1)$ \\
\hline $\mathrm{O}(1)$ & $9724(4)$ & $2179(2)$ & $2486(2)$ & $55(2)$ \\
\hline $\mathrm{O}(2)$ & $10702(3)$ & $3041(2)$ & $2985(2)$ & $50(1)$ \\
\hline $\mathrm{O}(3)$ & $9584(3)$ & $3366(2)$ & $3778(2)$ & $52(1)$ \\
\hline $\mathrm{O}(4)$ & $8181(3)$ & 1974(3) & $3278(2)$ & $63(2)$ \\
\hline $\mathrm{O}(5)$ & $9043(3)$ & 1913(3) & $4197(2)$ & $55(2)$ \\
\hline $\mathrm{O}(6)$ & $10652(3)$ & $1651(2)$ & $3787(2)$ & $43(1)$ \\
\hline $\mathrm{N}(1)$ & $9237(3)$ & $1575(2)$ & $5948(2)$ & $24(1)$ \\
\hline $\mathrm{N}(2)$ & $9308(3)$ & $613(2)$ & $6640(2)$ & $22(1)$ \\
\hline $\mathrm{N}(3)$ & $7675(3)$ & $303(2)$ & $6400(2)$ & $23(1)$ \\
\hline $\mathrm{N}(4)$ & $7614(3)$ & $1255(2)$ & $5695(2)$ & $23(1)$ \\
\hline $\mathrm{C}(1)$ & $9022(4)$ & 2183(3) & $5802(2)$ & $27(1)$ \\
\hline $\mathrm{C}(2)$ & $9762(4)$ & $2516(3)$ & $5707(3)$ & $37(2)$ \\
\hline $\mathrm{C}(3)$ & $10464(4)$ & 2097(3) & $5802(3)$ & $34(2)$ \\
\hline$C(4)$ & $10131(4)$ & $1523(3)$ & $5941(2)$ & $25(1)$ \\
\hline$C(5)$ & $10549(4)$ & $892(3)$ & $6047(2)$ & $26(1)$ \\
\hline $\mathrm{C}(6)$ & $10406(4)$ & $445(3)$ & $5573(2)$ & $29(1)$ \\
\hline$C(7)$ & $11530(4)$ & $986(3)$ & $6113(3)$ & $39(2)$ \\
\hline $\mathrm{C}(8)$ & $10182(3)$ & $602(3)$ & $6543(2)$ & $24(1)$ \\
\hline $\mathrm{C}(9)$ & $10572(4)$ & 271(3) & $6948(2)$ & $26(1)$ \\
\hline$C(10)$ & $9917(4)$ & $96(3)$ & $7310(2)$ & $22(1)$ \\
\hline$C(11)$ & $9153(4)$ & $301(3)$ & $7114(2)$ & $20(1)$ \\
\hline$C(12)$ & $8245(4)$ & $267(3)$ & $7329(2)$ & $25(1)$ \\
\hline$C(13)$ & $8224(4)$ & $-194(3)$ & $7804(3)$ & $33(2)$ \\
\hline$C(14)$ & $7969(4)$ & $934(3)$ & $7531(3)$ & $32(2)$ \\
\hline$C(15)$ & $7633(4)$ & $45(3)$ & $6903(2)$ & $22(1)$ \\
\hline$C(16)$ & $6959(4)$ & $-367(3)$ & $6926(3)$ & $30(2)$ \\
\hline$C(17)$ & $6571(4)$ & $-376(3)$ & $6415(3)$ & $36(2)$ \\
\hline$C(18)$ & $7019(3)$ & $36(3)$ & $6101(2)$ & $25(1)$ \\
\hline$C(19)$ & $6929(4)$ & $197(3)$ & $5519(2)$ & $29(1)$ \\
\hline$C(20)$ & $7669(4)$ & $-110(3)$ & $5204(3)$ & $35(2)$ \\
\hline $\mathrm{C}(21)$ & $6079(4)$ & $-60(3)$ & $5302(3)$ & $41(2)$ \\
\hline $\mathrm{C}(22)$ & $6968(4)$ & $902(3)$ & $5448(2)$ & $26(1)$ \\
\hline $\mathrm{C}(23)$ & $6481(4)$ & $1314(3)$ & $5154(3)$ & $39(2)$ \\
\hline$C(24)$ & $6838(5)$ & $1930(3)$ & $5214(3)$ & $40(2)$ \\
\hline$C(25)$ & $7532(4)$ & 1884(3) & $5547(2)$ & $30(2)$ \\
\hline
\end{tabular}




\begin{tabular}{rrrrr}
$\mathrm{C}(26)$ & $8089(4)$ & $2389(3)$ & $5794(3)$ & $30(2)$ \\
$\mathrm{C}(27)$ & $7991(5)$ & $3011(3)$ & $5483(3)$ & $53(2)$ \\
$\mathrm{C}(28)$ & $7782(4)$ & $2510(3)$ & $6368(3)$ & $40(2)$ \\
$\mathrm{C}(29)$ & $9648(6)$ & $1577(4)$ & $2244(4)$ & $75(3)$ \\
$\mathrm{C}(30)$ & $10186(5)$ & $2616(3)$ & $2188(3)$ & $41(2)$ \\
$\mathrm{C}(31)$ & $10341(6)$ & $3193(4)$ & $2503(3)$ & $59(2)$ \\
$\mathrm{C}(32)$ & $10875(5)$ & $3565(4)$ & $3323(3)$ & $49(2)$ \\
$\mathrm{C}(33)$ & $10100(5)$ & $3859(3)$ & $3566(3)$ & $46(2)$ \\
$\mathrm{C}(34)$ & $8928(7)$ & $3602(5)$ & $4104(4)$ & $91(4)$ \\
$\mathrm{C}(35)$ & $7634(6)$ & $2256(6)$ & $2890(5)$ & $96(4)$ \\
$\mathrm{C}(36)$ & $7731(5)$ & $1813(5)$ & $3747(4)$ & $70(3)$ \\
$\mathrm{C}(37)$ & $8345(6)$ & $1494(4)$ & $4108(4)$ & $70(3)$ \\
$\mathrm{C}(38)$ & $9713(7)$ & $1667(6)$ & $4509(4)$ & $100(4)$ \\
$\mathrm{C}(39)$ & $10369(7)$ & $1397(9)$ & $4259(5)$ & $163(8)$ \\
$\mathrm{C}(40)$ & $11145(6)$ & $1205(4)$ & $3494(5)$ & $88(4)$ \\
\hline
\end{tabular}

${ }^{a} U_{\text {eq }}$ is defined as one third of the trace of the orthogonalized $U_{i j}$ tensor. 
Table S4. Bond lengths (in $\AA$ ) for $\left[\mathrm{Na}(\text { diglyme })_{2}\right]\left[\mathrm{LFe}^{\mathrm{III}}\right]$.

\begin{tabular}{rrrr}
\hline Bond & Length & Bond & Length \\
\hline $\mathrm{Fe}(1)-\mathrm{N}(3)$ & $1.896(5)$ & $\mathrm{C}(1)-\mathrm{C}(26)$ & $1.513(9)$ \\
$\mathrm{Fe}(1)-\mathrm{N}(4)$ & $1.897(5)$ & $\mathrm{C}(2)-\mathrm{C}(3)$ & $1.424(9)$ \\
$\mathrm{Fe}(1)-\mathrm{N}(1)$ & $1.899(5)$ & $\mathrm{C}(3)-\mathrm{C}(4)$ & $1.362(8)$ \\
$\mathrm{Fe}(1)-\mathrm{N}(2)$ & $1.904(5)$ & $\mathrm{C}(4)-\mathrm{C}(5)$ & $1.507(9)$ \\
$\mathrm{Na}(1)-\mathrm{O}(3)$ & $2.341(5)$ & $\mathrm{C}(5)-\mathrm{C}(8)$ & $1.507(8)$ \\
$\mathrm{Na}(1)-\mathrm{O}(6)$ & $2.343(5)$ & $\mathrm{C}(5)-\mathrm{C}(6)$ & $1.539(8)$ \\
$\mathrm{Na}(1)-\mathrm{O}(1)$ & $2.357(6)$ & $\mathrm{C}(5)-\mathrm{C}(7)$ & $1.545(8)$ \\
$\mathrm{Na}(1)-\mathrm{O}(5)$ & $2.391(5)$ & $\mathrm{C}(9)-\mathrm{C}(10)$ & $1.418(8)$ \\
$\mathrm{Na}(1)-\mathrm{O}(2)$ & $2.432(5)$ & $\mathrm{C}(10)-\mathrm{C}(11)$ & $1.357(8)$ \\
$\mathrm{Na}(1)-\mathrm{O}(4)$ & $2.445(6)$ & $\mathrm{C}(11)-\mathrm{C}(12)$ & $1.514(8)$ \\
$\mathrm{O}(1)-\mathrm{C}(30)$ & $1.390(8)$ & $\mathrm{C}(12)-\mathrm{C}(15)$ & $1.511(8)$ \\
$\mathrm{O}(1)-\mathrm{C}(29)$ & $1.414(9)$ & $\mathrm{C}(12)-\mathrm{C}(13)$ & $1.545(8)$ \\
$\mathrm{O}(2)-\mathrm{C}(31)$ & $1.378(9)$ & $\mathrm{C}(12)-\mathrm{C}(14)$ & $1.558(8)$ \\
$\mathrm{O}(2)-\mathrm{C}(32)$ & $1.423(8)$ & $\mathrm{C}(15)-\mathrm{C}(16)$ & $1.363(8)$ \\
$\mathrm{O}(3)-\mathrm{C}(34)$ & $1.402(10)$ & $\mathrm{C}(16)-\mathrm{C}(17)$ & $1.424(9)$ \\
$\mathrm{O}(3)-\mathrm{C}(33)$ & $1.419(8)$ & $\mathrm{C}(17)-\mathrm{C}(18)$ & $1.368(9)$ \\
$\mathrm{O}(4)-\mathrm{C}(36)$ & $1.416(10)$ & $\mathrm{C}(18)-\mathrm{C}(19)$ & $1.513(9)$ \\
$\mathrm{O}(4)-\mathrm{C}(35)$ & $1.426(10)$ & $\mathrm{C}(19)-\mathrm{C}(22)$ & $1.499(9)$ \\
$\mathrm{O}(5)-\mathrm{C}(38)$ & $1.406(10)$ & $\mathrm{C}(19)-\mathrm{C}(21)$ & $1.530(8)$ \\
$\mathrm{O}(5)-\mathrm{C}(37)$ & $1.417(10)$ & $\mathrm{C}(19)-\mathrm{C}(20)$ & $1.542(8)$ \\
$\mathrm{O}(6)-\mathrm{C}(39)$ & $1.378(10)$ & $\mathrm{C}(22)-\mathrm{C}(23)$ & $1.372(9)$ \\
$\mathrm{O}(6)-\mathrm{C}(40)$ & $1.421(10)$ & $\mathrm{C}(23)-\mathrm{C}(24)$ & $1.422(10)$ \\
$\mathrm{N}(1)-\mathrm{C}(1)$ & $1.375(7)$ & $\mathrm{C}(24)-\mathrm{C}(25)$ & $1.370(9)$ \\
$\mathrm{N}(1)-\mathrm{C}(4)$ & $1.393(7)$ & $\mathrm{C}(25)-\mathrm{C}(26)$ & $1.508(9)$ \\
$\mathrm{N}(2)-\mathrm{C}(8)$ & $1.379(7)$ & $\mathrm{C}(26)-\mathrm{C}(27)$ & $1.537(9)$ \\
$\mathrm{N}(2)-\mathrm{C}(11)$ & $1.387(7)$ & $\mathrm{C}(26)-\mathrm{C}(28)$ & $1.549(9)$ \\
$\mathrm{N}(3)-\mathrm{C}(15)$ & $1.382(7)$ & $\mathrm{C}(30)-\mathrm{C}(31)$ & $1.474(10)$ \\
$\mathrm{N}(3)-\mathrm{C}(18)$ & $1.388(7)$ & $\mathrm{C}(32)-\mathrm{C}(33)$ & $1.487(10)$ \\
$\mathrm{N}(4)-\mathrm{C}(25)$ & $1.386(8)$ & $\mathrm{C}(36)-\mathrm{C}(37)$ & $1.483(13)$ \\
$\mathrm{N}(4)-\mathrm{C}(22)$ & $1.396(8)$ & $\mathrm{C}(38)-\mathrm{C}(39)$ & $1.328(15)$ \\
$\mathrm{C}(1)-\mathrm{C}(2)$ & $1.369(9)$ & & \\
\hline
\end{tabular}


Table S5. Bond angles (in deg) for $\left[\mathrm{Na}(\text { diglyme })_{2}\right]\left[\mathrm{LFe}^{\mathrm{III}}\right]$.

\begin{tabular}{|c|c|c|c|}
\hline Bond angle & Angle & Bond angle & Angle \\
\hline $\mathrm{N}(3)-\mathrm{Fe}(1)-\mathrm{N}(4)$ & $90.2(2)$ & $\mathrm{C}(40)-\mathrm{O}(6)-\mathrm{Na}(1)$ & $124.1(5)$ \\
\hline $\mathrm{N}(3)-\mathrm{Fe}(1)-\mathrm{N}(1)$ & $178.7(2)$ & $\mathrm{C}(1)-\mathrm{N}(1)-\mathrm{C}(4)$ & $108.2(5)$ \\
\hline $\mathrm{N}(4)-\mathrm{Fe}(1)-\mathrm{N}(1)$ & $90.2(2)$ & $\mathrm{C}(1)-\mathrm{N}(1)-\mathrm{Fe}(1)$ & $126.0(4)$ \\
\hline $\mathrm{N}(3)-\mathrm{Fe}(1)-\mathrm{N}(2)$ & $90.1(2)$ & $\mathrm{C}(4)-\mathrm{N}(1)-\mathrm{Fe}(1)$ & $125.7(4)$ \\
\hline $\mathrm{N}(4)-\mathrm{Fe}(1)-\mathrm{N}(2)$ & $179.8(2)$ & $\mathrm{C}(8)-\mathrm{N}(2)-\mathrm{C}(11)$ & $108.3(5)$ \\
\hline $\mathrm{N}(1)-\mathrm{Fe}(1)-\mathrm{N}(2)$ & $89.6(2)$ & $\mathrm{C}(8)-\mathrm{N}(2)-\mathrm{Fe}(1)$ & $125.3(4)$ \\
\hline $\mathrm{O}(3)-\mathrm{Na}(1)-\mathrm{O}(6)$ & $116.4(2)$ & $\mathrm{C}(11)-\mathrm{N}(2)-\mathrm{Fe}(1)$ & $126.1(4)$ \\
\hline $\mathrm{O}(3)-\mathrm{Na}(1)-\mathrm{O}(1)$ & $122.4(2)$ & $\mathrm{C}(15)-\mathrm{N}(3)-\mathrm{C}(18)$ & $107.9(5)$ \\
\hline $\mathrm{O}(6)-\mathrm{Na}(1)-\mathrm{O}(1)$ & $106.0(2)$ & $\mathrm{C}(15)-\mathrm{N}(3)-\mathrm{Fe}(1)$ & $126.2(4)$ \\
\hline $\mathrm{O}(3)-\mathrm{Na}(1)-\mathrm{O}(5)$ & $90.2(2)$ & $\mathrm{C}(18)-\mathrm{N}(3)-\mathrm{Fe}(1)$ & $125.9(4)$ \\
\hline $\mathrm{O}(6)-\mathrm{Na}(1)-\mathrm{O}(5)$ & $71.33(18)$ & $\mathrm{C}(25)-\mathrm{N}(4)-\mathrm{C}(22)$ & $109.0(5)$ \\
\hline $\mathrm{O}(1)-\mathrm{Na}(1)-\mathrm{O}(5)$ & $141.4(2)$ & $\mathrm{C}(25)-\mathrm{N}(4)-\mathrm{Fe}(1)$ & $125.1(4)$ \\
\hline $\mathrm{O}(3)-\mathrm{Na}(1)-\mathrm{O}(2)$ & $69.96(18)$ & $\mathrm{C}(22)-\mathrm{N}(4)-\mathrm{Fe}(1)$ & $125.9(4)$ \\
\hline $\mathrm{O}(6)-\mathrm{Na}(1)-\mathrm{O}(2)$ & $96.54(19)$ & $\mathrm{C}(2)-\mathrm{C}(1)-\mathrm{N}(1)$ & $108.8(6)$ \\
\hline $\mathrm{O}(1)-\mathrm{Na}(1)-\mathrm{O}(2)$ & $68.13(18)$ & $C(2)-C(1)-C(26)$ & $130.8(5)$ \\
\hline $\mathrm{O}(5)-\mathrm{Na}(1)-\mathrm{O}(2)$ & $149.6(2)$ & $\mathrm{N}(1)-\mathrm{C}(1)-\mathrm{C}(26)$ & $120.3(5)$ \\
\hline $\mathrm{O}(3)-\mathrm{Na}(1)-\mathrm{O}(4)$ & $107.9(2)$ & $\mathrm{C}(1)-\mathrm{C}(2)-\mathrm{C}(3)$ & $107.1(5)$ \\
\hline $\mathrm{O}(6)-\mathrm{Na}(1)-\mathrm{O}(4)$ & $118.0(2)$ & $C(4)-C(3)-C(2)$ & $107.7(6)$ \\
\hline $\mathrm{O}(1)-\mathrm{Na}(1)-\mathrm{O}(4)$ & $82.0(2)$ & $\mathrm{C}(3)-\mathrm{C}(4)-\mathrm{N}(1)$ & $108.2(6)$ \\
\hline $\mathrm{O}(5)-\mathrm{Na}(1)-\mathrm{O}(4)$ & $67.4(2)$ & $C(3)-C(4)-C(5)$ & $131.9(6)$ \\
\hline $\mathrm{O}(2)-\mathrm{Na}(1)-\mathrm{O}(4)$ & $139.6(2)$ & $\mathrm{N}(1)-\mathrm{C}(4)-\mathrm{C}(5)$ & $119.8(5)$ \\
\hline $\mathrm{C}(30)-\mathrm{O}(1)-\mathrm{C}(29)$ & $113.8(7)$ & $C(4)-C(5)-C(8)$ & $110.1(5)$ \\
\hline $\mathrm{C}(30)-\mathrm{O}(1)-\mathrm{Na}(1)$ & $117.2(4)$ & $C(4)-C(5)-C(6)$ & $109.9(5)$ \\
\hline $\mathrm{C}(29)-\mathrm{O}(1)-\mathrm{Na}(1)$ & $124.1(5)$ & $C(8)-C(5)-C(6)$ & $110.0(5)$ \\
\hline $\mathrm{C}(31)-\mathrm{O}(2)-\mathrm{C}(32)$ & $115.1(6)$ & $C(4)-C(5)-C(7)$ & $109.3(5)$ \\
\hline $\mathrm{C}(31)-\mathrm{O}(2)-\mathrm{Na}(1)$ & $104.8(4)$ & $C(8)-C(5)-C(7)$ & $109.7(5)$ \\
\hline $\mathrm{C}(32)-\mathrm{O}(2)-\mathrm{Na}(1)$ & $109.3(4)$ & $C(6)-C(5)-C(7)$ & $107.8(5)$ \\
\hline $\mathrm{C}(34)-\mathrm{O}(3)-\mathrm{C}(33)$ & $111.8(6)$ & $\mathrm{C}(9)-\mathrm{C}(8)-\mathrm{N}(2)$ & $108.1(5)$ \\
\hline $\mathrm{C}(34)-\mathrm{O}(3)-\mathrm{Na}(1)$ & $126.4(5)$ & $\mathrm{C}(9)-\mathrm{C}(8)-\mathrm{C}(5)$ & $131.0(5)$ \\
\hline $\mathrm{C}(33)-\mathrm{O}(3)-\mathrm{Na}(1)$ & $119.7(4)$ & $\mathrm{N}(2)-\mathrm{C}(8)-\mathrm{C}(5)$ & $120.9(5)$ \\
\hline $\mathrm{C}(36)-\mathrm{O}(4)-\mathrm{C}(35)$ & $112.3(7)$ & $C(8)-C(9)-C(10)$ & $107.1(5)$ \\
\hline $\mathrm{C}(36)-\mathrm{O}(4)-\mathrm{Na}(1)$ & $115.3(5)$ & $\mathrm{C}(11)-\mathrm{C}(10)-\mathrm{C}(9)$ & $108.0(5)$ \\
\hline $\mathrm{C}(35)-\mathrm{O}(4)-\mathrm{Na}(1)$ & $121.0(6)$ & $\mathrm{C}(10)-\mathrm{C}(11)-\mathrm{N}(2)$ & $108.4(5)$ \\
\hline $\mathrm{C}(38)-\mathrm{O}(5)-\mathrm{C}(37)$ & $115.1(8)$ & $\mathrm{C}(10)-\mathrm{C}(11)-\mathrm{C}(12)$ & $131.9(5)$ \\
\hline $\mathrm{C}(38)-\mathrm{O}(5)-\mathrm{Na}(1)$ & $108.4(5)$ & $\mathrm{N}(2)-\mathrm{C}(11)-\mathrm{C}(12)$ & $119.6(5)$ \\
\hline $\mathrm{C}(37)-\mathrm{O}(5)-\mathrm{Na}(1)$ & $114.4(5)$ & $C(15)-C(12)-C(11)$ & $110.2(5)$ \\
\hline $\mathrm{C}(39)-\mathrm{O}(6)-\mathrm{C}(40)$ & $111.3(10)$ & $\mathrm{C}(15)-\mathrm{C}(12)-\mathrm{C}(13)$ & $110.2(5)$ \\
\hline $\mathrm{C}(39)-\mathrm{O}(6)-\mathrm{Na}(1)$ & $112.8(6)$ & $C(11)-C(12)-C(13)$ & $109.2(5)$ \\
\hline
\end{tabular}




\begin{tabular}{rrrr}
$\mathrm{C}(15)-\mathrm{C}(12)-\mathrm{C}(14)$ & $109.8(5)$ & $\mathrm{C}(22)-\mathrm{C}(23)-\mathrm{C}(24)$ & $107.9(6)$ \\
$\mathrm{C}(11)-\mathrm{C}(12)-\mathrm{C}(14)$ & $109.4(5)$ & $\mathrm{C}(25)-\mathrm{C}(24)-\mathrm{C}(23)$ & $107.9(6)$ \\
$\mathrm{C}(13)-\mathrm{C}(12)-\mathrm{C}(14)$ & $108.0(5)$ & $\mathrm{C}(24)-\mathrm{C}(25)-\mathrm{N}(4)$ & $107.8(6)$ \\
$\mathrm{C}(16)-\mathrm{C}(15)-\mathrm{N}(3)$ & $109.0(5)$ & $\mathrm{C}(24)-\mathrm{C}(25)-\mathrm{C}(26)$ & $130.9(6)$ \\
$\mathrm{C}(16)-\mathrm{C}(15)-\mathrm{C}(12)$ & $130.6(6)$ & $\mathrm{N}(4)-\mathrm{C}(25)-\mathrm{C}(26)$ & $120.9(5)$ \\
$\mathrm{N}(3)-\mathrm{C}(15)-\mathrm{C}(12)$ & $120.2(5)$ & $\mathrm{C}(25)-\mathrm{C}(26)-\mathrm{C}(1)$ & $110.7(5)$ \\
$\mathrm{C}(15)-\mathrm{C}(16)-\mathrm{C}(17)$ & $107.2(6)$ & $\mathrm{C}(25)-\mathrm{C}(26)-\mathrm{C}(27)$ & $109.6(5)$ \\
$\mathrm{C}(18)-\mathrm{C}(17)-\mathrm{C}(16)$ & $107.5(5)$ & $\mathrm{C}(1)-\mathrm{C}(26)-\mathrm{C}(27)$ & $110.4(6)$ \\
$\mathrm{C}(17)-\mathrm{C}(18)-\mathrm{N}(3)$ & $108.4(5)$ & $\mathrm{C}(25)-\mathrm{C}(26)-\mathrm{C}(28)$ & $109.0(5)$ \\
$\mathrm{C}(17)-\mathrm{C}(18)-\mathrm{C}(19)$ & $131.1(6)$ & $\mathrm{C}(1)-\mathrm{C}(26)-\mathrm{C}(28)$ & $109.3(5)$ \\
$\mathrm{N}(3)-\mathrm{C}(18)-\mathrm{C}(19)$ & $120.4(5)$ & $\mathrm{C}(27)-\mathrm{C}(26)-\mathrm{C}(28)$ & $107.8(6)$ \\
$\mathrm{C}(22)-\mathrm{C}(19)-\mathrm{C}(18)$ & $109.5(5)$ & $\mathrm{O}(1)-\mathrm{C}(30)-\mathrm{C}(31)$ & $109.9(6)$ \\
$\mathrm{C}(22)-\mathrm{C}(19)-\mathrm{C}(21)$ & $110.1(5)$ & $\mathrm{O}(2)-\mathrm{C}(31)-\mathrm{C}(30)$ & $110.5(6)$ \\
$\mathrm{C}(18)-\mathrm{C}(19)-\mathrm{C}(21)$ & $110.4(5)$ & $\mathrm{O}(2)-\mathrm{C}(32)-\mathrm{C}(33)$ & $114.8(6)$ \\
$\mathrm{C}(22)-\mathrm{C}(19)-\mathrm{C}(20)$ & $109.0(5)$ & $\mathrm{O}(3)-\mathrm{C}(33)-\mathrm{C}(32)$ & $107.9(6)$ \\
$\mathrm{C}(18)-\mathrm{C}(19)-\mathrm{C}(20)$ & $109.7(5)$ & $\mathrm{O}(4)-\mathrm{C}(36)-\mathrm{C}(37)$ & $107.8(7)$ \\
$\mathrm{C}(21)-\mathrm{C}(19)-\mathrm{C}(20)$ & $108.0(5)$ & $\mathrm{O}(5)-\mathrm{C}(37)-\mathrm{C}(36)$ & $107.8(7)$ \\
$\mathrm{C}(23)-\mathrm{C}(22)-\mathrm{N}(4)$ & $107.4(5)$ & $\mathrm{C}(39)-\mathrm{C}(38)-\mathrm{O}(5)$ & $117.3(8)$ \\
$\mathrm{C}(23)-\mathrm{C}(22)-\mathrm{C}(19)$ & $132.2(6)$ & $\mathrm{C}(38)-\mathrm{C}(39)-\mathrm{O}(6)$ & $119.3(11)$ \\
$\mathrm{N}(4)-\mathrm{C}(22)-\mathrm{C}(19)$ & $120.3(5)$ & & \\
\hline
\end{tabular}


Table S6. Anisotropic displacement parameters $\left(\AA^{2} \times 10^{3}\right)$ for $\left[\mathrm{Na}(\text { diglyme })_{2}\right]\left[\mathrm{LFe}^{\mathrm{III}}\right]$.

\begin{tabular}{|c|c|c|c|c|c|c|}
\hline Atom & $\mathrm{U}_{11}$ & $\mathrm{U}_{22}$ & $\mathrm{U}_{33}$ & $\mathrm{U}_{23}$ & $\mathrm{U}_{13}$ & $\mathrm{U}_{12}$ \\
\hline $\mathrm{Fe}(1)$ & $17(1)$ & $22(1)$ & $24(1)$ & $4(1)$ & $-2(1)$ & $0(1)$ \\
\hline $\mathrm{Na}(1)$ & $37(2)$ & $33(1)$ & $40(2)$ & $8(1)$ & $1(1)$ & $-4(1)$ \\
\hline $\mathrm{O}(1)$ & $86(4)$ & $40(3)$ & $40(3)$ & $-2(2)$ & $-3(3)$ & $-24(3)$ \\
\hline $\mathrm{O}(2)$ & $54(3)$ & $47(3)$ & $47(3)$ & $-7(3)$ & $-2(3)$ & $-17(3)$ \\
\hline $\mathrm{O}(3)$ & $51(3)$ & $46(3)$ & $59(3)$ & $-12(3)$ & $19(3)$ & $-4(2)$ \\
\hline $\mathrm{O}(4)$ & $32(3)$ & $88(4)$ & $71(4)$ & $13(3)$ & $-1(3)$ & $-2(3)$ \\
\hline $\mathrm{O}(5)$ & $40(3)$ & $71(4)$ & $53(3)$ & $24(3)$ & $15(3)$ & $15(3)$ \\
\hline $\mathrm{O}(6)$ & $42(3)$ & $43(3)$ & $45(3)$ & $15(2)$ & $9(2)$ & $8(2)$ \\
\hline $\mathrm{N}(1)$ & $25(3)$ & $21(3)$ & $27(3)$ & $-1(2)$ & $1(2)$ & $-2(2)$ \\
\hline $\mathrm{N}(2)$ & $11(2)$ & $28(3)$ & $27(3)$ & $3(2)$ & $-2(2)$ & $0(2)$ \\
\hline $\mathrm{N}(3)$ & $13(2)$ & $24(3)$ & $31(3)$ & $1(2)$ & $3(2)$ & $-1(2)$ \\
\hline $\mathrm{N}(4)$ & $24(3)$ & $21(3)$ & $23(3)$ & $-2(2)$ & $-7(2)$ & $4(2)$ \\
\hline $\mathrm{C}(1)$ & $38(4)$ & $19(3)$ & $25(3)$ & $4(3)$ & $2(3)$ & $0(3)$ \\
\hline$C(2)$ & $53(4)$ & $23(3)$ & $36(4)$ & $8(3)$ & $7(3)$ & $-9(3)$ \\
\hline$C(3)$ & $28(4)$ & $37(4)$ & $36(4)$ & $0(3)$ & $11(3)$ & $-15(3)$ \\
\hline$C(4)$ & $23(3)$ & $34(3)$ & $18(3)$ & $-4(3)$ & $2(3)$ & $-6(3)$ \\
\hline$C(5)$ & $19(3)$ & $35(3)$ & $23(3)$ & $-2(3)$ & $1(2)$ & $-3(3)$ \\
\hline $\mathrm{C}(6)$ & $22(3)$ & $32(3)$ & $33(4)$ & $-6(3)$ & $6(3)$ & $1(3)$ \\
\hline$C(7)$ & $18(3)$ & $56(4)$ & $43(4)$ & $2(4)$ & $2(3)$ & $-11(3)$ \\
\hline $\mathrm{C}(8)$ & $14(3)$ & $28(3)$ & $29(3)$ & $-3(3)$ & $-2(3)$ & $7(2)$ \\
\hline $\mathrm{C}(9)$ & $16(3)$ & $31(3)$ & $32(3)$ & $-2(3)$ & $-7(3)$ & $2(3)$ \\
\hline $\mathrm{C}(10)$ & $22(3)$ & $22(3)$ & $21(3)$ & $4(2)$ & $-5(3)$ & $3(2)$ \\
\hline $\mathrm{C}(11)$ & $25(3)$ & $17(3)$ & $17(3)$ & $-2(2)$ & $-2(2)$ & $-1(2)$ \\
\hline $\mathrm{C}(12)$ & $22(3)$ & $30(3)$ & $24(3)$ & $8(3)$ & $3(3)$ & $2(3)$ \\
\hline $\mathrm{C}(13)$ & $28(3)$ & $34(4)$ & $37(4)$ & $9(3)$ & $4(3)$ & $-1(3)$ \\
\hline$C(14)$ & $22(3)$ & $44(4)$ & $28(3)$ & $0(3)$ & $1(3)$ & $6(3)$ \\
\hline $\mathrm{C}(15)$ & $18(3)$ & $22(3)$ & $26(3)$ & $0(3)$ & $-2(3)$ & $2(2)$ \\
\hline$C(16)$ & $21(3)$ & $29(3)$ & $41(4)$ & $1(3)$ & $8(3)$ & $-8(3)$ \\
\hline $\mathrm{C}(17)$ & $21(3)$ & $37(4)$ & $48(4)$ & $-12(3)$ & $1(3)$ & $-10(3)$ \\
\hline $\mathrm{C}(18)$ & $12(3)$ & $27(3)$ & $37(4)$ & $-9(3)$ & $2(3)$ & $2(2)$ \\
\hline C(19) & $17(3)$ & $37(4)$ & $31(4)$ & $-8(3)$ & $-3(3)$ & $-1(3)$ \\
\hline $\mathrm{C}(20)$ & $30(4)$ & $40(4)$ & $35(4)$ & $-10(3)$ & $-2(3)$ & $6(3)$ \\
\hline $\mathrm{C}(21)$ & $25(4)$ & $47(4)$ & $50(4)$ & $-13(4)$ & $-12(3)$ & $-1(3)$ \\
\hline $\mathrm{C}(22)$ & $24(3)$ & $32(3)$ & $23(3)$ & $-6(3)$ & $-1(3)$ & $8(3)$ \\
\hline $\mathrm{C}(23)$ & $33(4)$ & $48(4)$ & $37(4)$ & $-3(3)$ & $-17(3)$ & $6(3)$ \\
\hline $\mathrm{C}(24)$ & $45(4)$ & $37(4)$ & $38(4)$ & $10(3)$ & $-7(3)$ & $11(3)$ \\
\hline $\mathrm{C}(25)$ & $38(4)$ & $32(3)$ & $20(3)$ & $1(3)$ & $5(3)$ & $11(3)$ \\
\hline$C(26)$ & $36(4)$ & $19(3)$ & $35(4)$ & $5(3)$ & $-1(3)$ & $5(3)$ \\
\hline
\end{tabular}




\begin{tabular}{lrrrrrr}
$\mathrm{C}(27)$ & $64(5)$ & $31(4)$ & $64(5)$ & $15(4)$ & $-8(4)$ & $7(4)$ \\
$\mathrm{C}(28)$ & $39(4)$ & $35(4)$ & $46(4)$ & $-12(3)$ & $-4(3)$ & $10(3)$ \\
$\mathrm{C}(29)$ & $86(7)$ & $41(5)$ & $97(7)$ & $-17(5)$ & $-33(6)$ & $-5(5)$ \\
$\mathrm{C}(30)$ & $48(4)$ & $42(4)$ & $34(4)$ & $4(3)$ & $4(3)$ & $-4(3)$ \\
$\mathrm{C}(31)$ & $83(6)$ & $44(4)$ & $51(5)$ & $20(4)$ & $-22(5)$ & $-20(4)$ \\
$\mathrm{C}(32)$ & $46(5)$ & $56(5)$ & $46(4)$ & $-13(4)$ & $0(4)$ & $-16(4)$ \\
$\mathrm{C}(33)$ & $41(4)$ & $36(4)$ & $62(5)$ & $-4(4)$ & $-10(4)$ & $-12(3)$ \\
$\mathrm{C}(34)$ & $93(8)$ & $77(7)$ & $103(8)$ & $-23(6)$ & $51(7)$ & $-15(6)$ \\
$\mathrm{C}(35)$ & $50(6)$ & $126(10)$ & $111(9)$ & $6(8)$ & $-32(6)$ & $13(6)$ \\
$\mathrm{C}(36)$ & $35(5)$ & $81(6)$ & $95(7)$ & $-3(6)$ & $18(5)$ & $-16(4)$ \\
$\mathrm{C}(37)$ & $70(6)$ & $59(5)$ & $82(7)$ & $17(5)$ & $38(5)$ & $1(5)$ \\
$\mathrm{C}(38)$ & $101(9)$ & $162(11)$ & $37(5)$ & $38(6)$ & $17(5)$ & $67(8)$ \\
$\mathrm{C}(39)$ & $56(7)$ & $320(20)$ & $113(10)$ & $162(13)$ & $17(7)$ & $36(10)$ \\
$\mathrm{C}(40)$ & $66(6)$ & $60(6)$ & $139(10)$ & $-55(6)$ & $-40(7)$ & $15(5)$ \\
\hline
\end{tabular}

The anisotropic displacement factor exponent takes the form: $-2 \pi^{2}\left[\mathrm{~h}^{2} \mathrm{a}^{{ }^{2}} \mathrm{U}_{11}+\ldots+\right.$ $\left.2 \mathrm{hka} * \mathrm{~b} * \mathrm{U}_{12}\right]$ 
Table S7. Hydrogen coordinates $\left(\times 10^{4}\right)$ and isotropic displacement parameters $\left(\AA^{2} \times 10^{3}\right)$ for $\left[\mathrm{Na}(\text { diglyme })_{2}\right]$ $\left[\mathrm{LFe}^{\mathrm{III}}\right]$.

\begin{tabular}{|c|c|c|c|c|}
\hline Atom & $\mathrm{x}$ & $\mathrm{y}$ & $\mathrm{Z}$ & $\mathrm{U}(\mathrm{eq})$ \\
\hline $\mathrm{H}(2)$ & 9800 & 2946 & 5598 & 45 \\
\hline $\mathrm{H}(3)$ & 11057 & 2199 & 5773 & 40 \\
\hline $\mathrm{H}(6 \mathrm{~A})$ & 9787 & 372 & 5526 & 43 \\
\hline $\mathrm{H}(6 \mathrm{~B})$ & 10695 & 40 & 5640 & 43 \\
\hline $\mathrm{H}(6 \mathrm{C})$ & 10643 & 638 & 5252 & 43 \\
\hline $\mathrm{H}(7 \mathrm{~A})$ & 11769 & 1165 & 5786 & 59 \\
\hline $\mathrm{H}(7 \mathrm{~B})$ & 11803 & 577 & 6186 & 59 \\
\hline $\mathrm{H}(7 \mathrm{C})$ & 11639 & 1277 & 6408 & 59 \\
\hline $\mathrm{H}(9)$ & 11168 & 178 & 6979 & 32 \\
\hline $\mathrm{H}(10)$ & 9998 & -126 & 7634 & 26 \\
\hline $\mathrm{H}(13 \mathrm{~A})$ & 7643 & -204 & 7955 & 49 \\
\hline $\mathrm{H}(13 \mathrm{~B})$ & 8633 & -51 & 8075 & 49 \\
\hline $\mathrm{H}(13 \mathrm{C})$ & 8384 & -620 & 7685 & 49 \\
\hline $\mathrm{H}(14 \mathrm{~A})$ & 7999 & 1239 & 7239 & 47 \\
\hline $\mathrm{H}(14 \mathrm{~B})$ & 8356 & 1069 & 7816 & 47 \\
\hline $\mathrm{H}(14 \mathrm{C})$ & 7378 & 914 & 7666 & 47 \\
\hline $\mathrm{H}(16)$ & 6781 & -604 & 7226 & 36 \\
\hline $\mathrm{H}(17)$ & 6088 & -623 & 6311 & 43 \\
\hline $\mathrm{H}(20 \mathrm{~A})$ & 7621 & 8 & 4830 & 53 \\
\hline $\mathrm{H}(20 \mathrm{~B})$ & 7635 & -572 & 5238 & 53 \\
\hline $\mathrm{H}(20 \mathrm{C})$ & 8222 & 38 & 5343 & 53 \\
\hline $\mathrm{H}(21 \mathrm{~A})$ & 5598 & 123 & 5502 & 61 \\
\hline $\mathrm{H}(21 \mathrm{~B})$ & 6069 & -522 & 5338 & 61 \\
\hline $\mathrm{H}(21 \mathrm{C})$ & 6024 & 55 & 4928 & 61 \\
\hline $\mathrm{H}(23)$ & 5992 & 1207 & 4947 & 47 \\
\hline $\mathrm{H}(24)$ & 6632 & 2307 & 5052 & 48 \\
\hline $\mathrm{H}(27 \mathrm{~A})$ & 8140 & 2940 & 5111 & 79 \\
\hline $\mathrm{H}(27 \mathrm{~B})$ & 8375 & 3333 & 5633 & 79 \\
\hline $\mathrm{H}(27 \mathrm{C})$ & 7394 & 3159 & 5508 & 79 \\
\hline $\mathrm{H}(28 \mathrm{~A})$ & 7167 & 2616 & 6366 & 60 \\
\hline $\mathrm{H}(28 \mathrm{~B})$ & 8108 & 2864 & 6521 & 60 \\
\hline $\mathrm{H}(28 \mathrm{C})$ & 7875 & 2128 & 6581 & 60 \\
\hline $\mathrm{H}(29 \mathrm{~A})$ & 9412 & 1627 & 1887 & 112 \\
\hline $\mathrm{H}(29 B)$ & 9264 & 1309 & 2455 & 112 \\
\hline $\mathrm{H}(29 \mathrm{C})$ & 10217 & 1379 & 2222 & 112 \\
\hline $\mathrm{H}(30 \mathrm{~A})$ & 9859 & 2725 & 1864 & 49 \\
\hline
\end{tabular}




\begin{tabular}{rrrrr}
$\mathrm{H}(30 \mathrm{~B})$ & 10743 & 2429 & 2079 & 49 \\
$\mathrm{H}(31 \mathrm{~A})$ & 10732 & 3479 & 2307 & 71 \\
$\mathrm{H}(31 \mathrm{~B})$ & 9790 & 3418 & 2560 & 71 \\
$\mathrm{H}(32 \mathrm{~A})$ & 11183 & 3893 & 3116 & 59 \\
$\mathrm{H}(32 \mathrm{~B})$ & 11264 & 3424 & 3610 & 59 \\
$\mathrm{H}(33 \mathrm{~A})$ & 10273 & 4156 & 3850 & 56 \\
$\mathrm{H}(33 \mathrm{~B})$ & 9773 & 4099 & 3296 & 56 \\
$\mathrm{H}(34 \mathrm{~A})$ & 9183 & 3810 & 4413 & 137 \\
$\mathrm{H}(34 \mathrm{~B})$ & 8560 & 3252 & 4221 & 137 \\
$\mathrm{H}(34 \mathrm{C})$ & 8582 & 3909 & 3905 & 137 \\
$\mathrm{H}(35 \mathrm{~A})$ & 7178 & 1957 & 2793 & 143 \\
$\mathrm{H}(35 \mathrm{~B})$ & 7973 & 2362 & 2574 & 143 \\
$\mathrm{H}(35 \mathrm{C})$ & 7376 & 2643 & 3035 & 143 \\
$\mathrm{H}(36 \mathrm{~A})$ & 7245 & 1527 & 3663 & 84 \\
$\mathrm{H}(36 \mathrm{~B})$ & 7497 & 2200 & 3916 & 84 \\
$\mathrm{H}(37 \mathrm{~A})$ & 8059 & 1391 & 4448 & 84 \\
$\mathrm{H}(37 \mathrm{~B})$ & 8554 & 1095 & 3947 & 84 \\
$\mathrm{H}(38 \mathrm{~A})$ & 9944 & 2016 & 4730 & 120 \\
$\mathrm{H}(38 \mathrm{~B})$ & 9463 & 1349 & 4754 & 120 \\
$\mathrm{H}(39 \mathrm{~A})$ & 10212 & 950 & 4191 & 196 \\
$\mathrm{H}(39 \mathrm{~B})$ & 10865 & 1393 & 4505 & 196 \\
$\mathrm{H}(40 \mathrm{~A})$ & 11679 & 1111 & 3686 & 133 \\
$\mathrm{H}(40 \mathrm{~B})$ & 11285 & 1383 & 3146 & 133 \\
$\mathrm{H}(40 \mathrm{C})$ & 10813 & 814 & 3449 & 133 \\
\hline
\end{tabular}



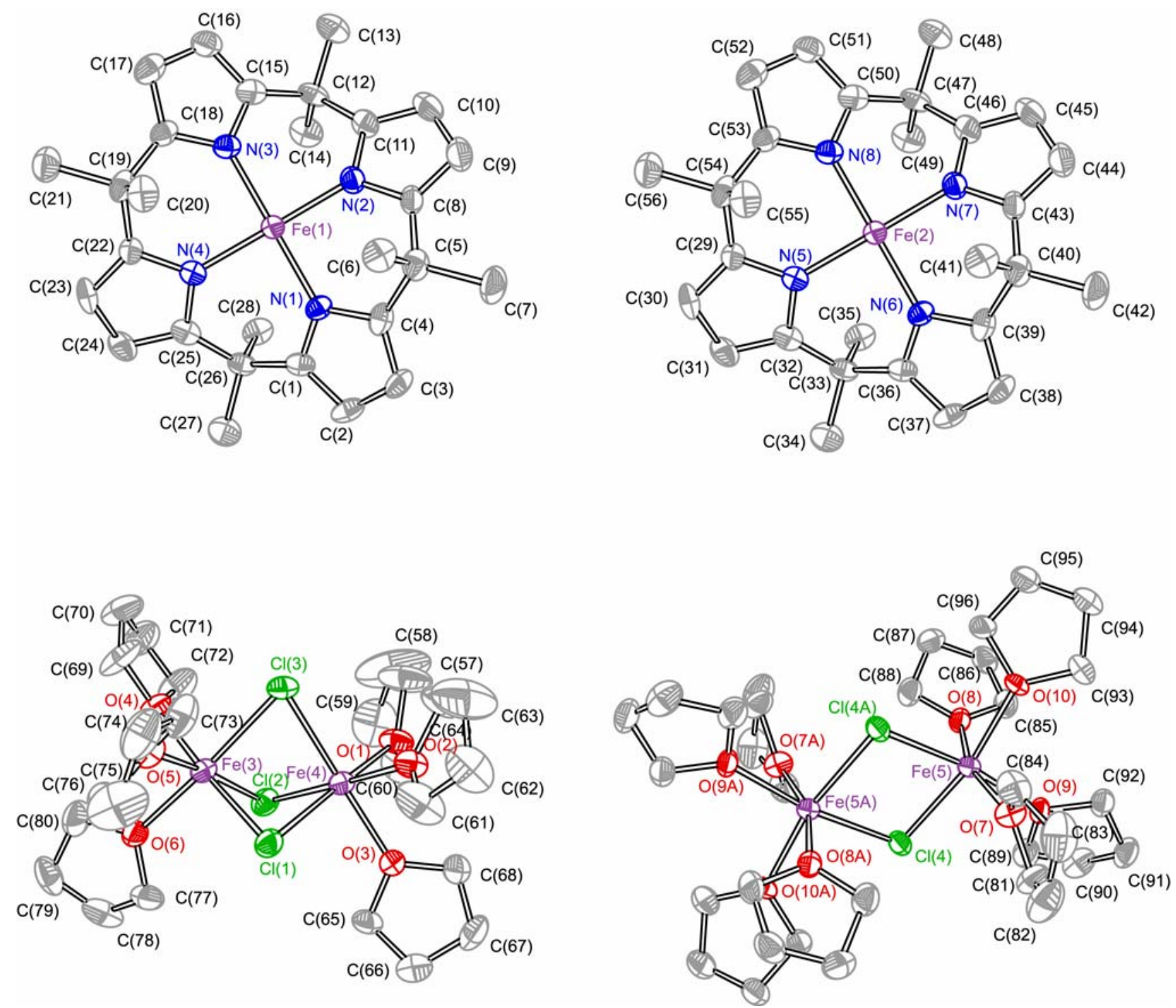

Figure S4. Atom labeling scheme for the individual components of $\left[(\mathrm{THF})_{3} \mathrm{Fe}^{\mathrm{II}}(\mu-\mathrm{Cl})_{3} \mathrm{Fe}^{\mathrm{II}}(\mathrm{THF})_{3}\right]\left[(\mathrm{THF})_{4} \mathrm{Fe}^{\mathrm{II}}(\mu-\mathrm{Cl})_{2} \mathrm{Fe}^{\mathrm{II}}(\mathrm{THF})_{4}\right]_{1 / 2}$ $\left[\mathrm{LFe}^{\mathrm{III}}\right]_{2} \bullet 2 \mathrm{PhCH}_{3}$. 
Table S8. Crystal data and structure refinement for $\left[(\mathrm{THF})_{3} \mathrm{Fe}^{\mathrm{II}}(\mu-\right.$ $\left.\mathrm{Cl})_{3} \mathrm{Fe}^{\mathrm{II}}(\mathrm{THF})_{3}\right]\left[(\mathrm{THF})_{4} \mathrm{Fe}^{\mathrm{III}}(\mu-\mathrm{Cl})_{2} \mathrm{Fe}^{\mathrm{II}}(\mathrm{THF})_{4}\right]_{1 / 2}\left[\mathrm{LFe}^{\mathrm{III}}\right]_{2} \cdot 2 \mathrm{PhCH}_{3}$.

\begin{tabular}{|c|c|}
\hline Empirical formula & $\mathrm{C}_{110} \mathrm{H}_{154} \mathrm{Cl}_{4} \mathrm{Fe}_{5} \mathrm{~N}_{8} \mathrm{O}_{10}$ \\
\hline Formula weight & 2169.53 \\
\hline$T(\mathrm{~K})$ & $183(2)$ \\
\hline$\lambda(\AA)$ & 0.71073 \\
\hline Crystal system & Triclinic \\
\hline Space group & $P \overline{1}$ \\
\hline$a(\AA)$ & $13.0139(8)$ \\
\hline$b(\AA)$ & $15.3786(10)$ \\
\hline$c(\AA)$ & $29.1680(19)$ \\
\hline$\alpha\left(^{\circ}\right)$ & $84.7050(10)$ \\
\hline$\beta\left(\left(^{\circ}\right)\right.$ & $80.1510(10)$ \\
\hline$\gamma\left({ }^{\circ}\right)$ & $69.8640(10)$ \\
\hline$V / \AA^{3}$ & $5396.5(6)$ \\
\hline$Z$ & 2 \\
\hline$\rho_{\text {calcd }}\left(\mathrm{g} / \mathrm{cm}^{3}\right)$ & 1.335 \\
\hline Absorption coefficient $\left(\mathrm{mm}^{-1}\right)$ & 0.816 \\
\hline$F(000)$ & 2296 \\
\hline Crystal size $\left(\mathrm{mm}^{3}\right)$ & $0.35 \times 0.18 \times 0.08$ \\
\hline$\theta$ range for data collection & 2.04 to $23.30^{\circ}$ \\
\hline Index ranges & $-14 \leq h \leq 13,-17 \leq k \leq 8,-32 \leq \ell \leq 32$ \\
\hline No. of coll. reflns & 22060 \\
\hline No. of ind. reflns $\left(\mathrm{R}_{\text {int }}\right)$ & $15256(0.0587)$ \\
\hline Completeness to $\theta=23.30^{\circ}$ & $97.9 \%$ \\
\hline Absorption correction & None \\
\hline Refinement method & Full-matrix least-squares on $F^{2}$ \\
\hline Data / restraints / parameters & $15256 / 0 / 1279$ \\
\hline $\mathrm{GOF}^{a}$ on $F^{2}$ & 1.429 \\
\hline$R 1,{ }^{b} \mathrm{w} R 2^{c}(\mathrm{I}>2 \sigma)$ & $0.0987,0.1812$ \\
\hline$R 1,{ }^{b} \mathrm{w} R 2^{c}$ (all data) & $0.1033,0.1831$ \\
\hline Extinction coefficient & $0.00000(15)$ \\
\hline Largest diff. peak and hole (e. $\AA^{-3}$ ) & 0.578 and -0.412 \\
\hline
\end{tabular}

${ }^{a} \mathrm{GOF}=\left(\Sigma w\left(F_{\mathrm{o}}{ }^{2}-F_{\mathrm{c}}{ }^{2}\right)^{2} /(n-p)\right)^{1 / 2}$ where $n$ is the number of data and $p$ is the number of parameters refined. ${ }^{b} R 1=\Sigma|| F_{\mathrm{o}}-\left|F_{\mathrm{c}}\right||\Sigma| F_{\mathrm{o}} \mid{ }^{c}{ }^{c} \mathrm{w} R 2=\left(\Sigma\left(w\left(F_{\mathrm{o}}{ }^{2}-\right.\right.\right.$ $\left.\left.F_{\mathrm{c}}{ }^{2}\right) / \Sigma\left(w\left(F_{\mathrm{o}}\right)^{2}\right)\right)^{1 / 2}$. 
Table S9. Atomic coordinates $\left(\times 10^{4}\right)$ and equivalent isotropic displacement parameters $\left(\AA^{2} \times 10^{3}\right)$ for $\left[(\mathrm{THF})_{3} \mathrm{Fe}^{\mathrm{II}}(\mu-\mathrm{Cl})_{3} \mathrm{Fe}^{\mathrm{II}}(\mathrm{THF})_{3}\right]\left[(\mathrm{THF})_{4} \mathrm{Fe}^{\mathrm{II}}(\mu-\right.$ $\left.\mathrm{Cl})_{2} \mathrm{Fe}^{\mathrm{II}}(\mathrm{THF})_{4}\right]_{1 / 2}\left[\mathrm{LFe}^{\mathrm{III}}\right]_{2} \cdot 2 \mathrm{PhCH}_{3}$.

\begin{tabular}{|c|c|c|c|c|}
\hline Atom & $\mathrm{X}$ & $\mathrm{y}$ & z & $\mathrm{U}_{\mathrm{eq}}{ }^{a}$ \\
\hline $\mathrm{Fe}(1)$ & $8824(1)$ & $5730(1)$ & $8456(1)$ & $24(1)$ \\
\hline $\mathrm{Fe}(2)$ & $6107(1)$ & $1413(1)$ & $5942(1)$ & $23(1)$ \\
\hline $\mathrm{Fe}(3)$ & $2202(1)$ & $10401(1)$ & $8519(1)$ & $31(1)$ \\
\hline $\mathrm{Fe}(4)$ & $2566(1)$ & $8732(1)$ & $9182(1)$ & $31(1)$ \\
\hline $\mathrm{Fe}(5)$ & $8981(1)$ & $5599(1)$ & $5499(1)$ & $28(1)$ \\
\hline $\mathrm{Cl}(1)$ & $2462(2)$ & $8829(1)$ & $8323(1)$ & $39(1)$ \\
\hline $\mathrm{Cl}(2)$ & $3770(2)$ & $9689(1)$ & $8973(1)$ & $39(1)$ \\
\hline $\mathrm{Cl}(3)$ & $967(2)$ & $10193(1)$ & $9238(1)$ & $42(1)$ \\
\hline $\mathrm{Cl}(4)$ & 10989(1) & $5009(1)$ & $5267(1)$ & $36(1)$ \\
\hline $\mathrm{O}(1)$ & $2804(4)$ & $8644(4)$ & $9900(2)$ & $45(1)$ \\
\hline $\mathrm{O}(2)$ & $1474(4)$ & $7951(4)$ & $9338(2)$ & $39(1)$ \\
\hline $\mathrm{O}(3)$ & $3852(4)$ & $7440(3)$ & $9113(2)$ & $40(1)$ \\
\hline $\mathrm{O}(4)$ & $1897(4)$ & $11756(3)$ & $8727(2)$ & $43(1)$ \\
\hline $\mathrm{O}(5)$ & $895(4)$ & $10833(3)$ & $8077(2)$ & $42(1)$ \\
\hline $\mathrm{O}(6)$ & $3282(4)$ & $10696(3)$ & $7946(2)$ & $41(1)$ \\
\hline $\mathrm{O}(7)$ & $8799(4)$ & $4396(3)$ & $5884(2)$ & $38(1)$ \\
\hline $\mathrm{O}(8)$ & $8840(4)$ & $7010(3)$ & $5311(2)$ & $35(1)$ \\
\hline $\mathrm{O}(9)$ & $9183(4)$ & $5968(3)$ & $6182(2)$ & $31(1)$ \\
\hline $\mathrm{O}(10)$ & $7188(4)$ & $6140(4)$ & $5555(2)$ & $36(1)$ \\
\hline $\mathrm{N}(1)$ & $7863(4)$ & $6619(4)$ & $8886(2)$ & $25(1)$ \\
\hline $\mathrm{N}(2)$ & $9486(5)$ & $6604(4)$ & $8173(2)$ & $29(1)$ \\
\hline $\mathrm{N}(3)$ & $9803(4)$ & $4829(4)$ & $8028(2)$ & $28(1)$ \\
\hline $\mathrm{N}(4)$ & $8182(4)$ & $4838(4)$ & $8740(2)$ & $26(1)$ \\
\hline $\mathrm{N}(5)$ & $4539(4)$ & $1827(4)$ & $6105(2)$ & $26(1)$ \\
\hline $\mathrm{N}(6)$ & $6004(4)$ & $2556(4)$ & $5606(2)$ & $26(1)$ \\
\hline $\mathrm{N}(7)$ & $7675(4)$ & $1006(4)$ & $5783(2)$ & $26(1)$ \\
\hline $\mathrm{N}(8)$ & $6206(4)$ & $266(4)$ & $6271(2)$ & $26(1)$ \\
\hline $\mathrm{C}(1)$ & $7496(6)$ & $7576(4)$ & $8808(2)$ & $29(2)$ \\
\hline $\mathrm{C}(2)$ & $6866(6)$ & $7971(5)$ & $9209(3)$ & $35(2)$ \\
\hline$C(3)$ & $6858(6)$ & $7257(5)$ & $9549(2)$ & $36(2)$ \\
\hline $\mathrm{C}(4)$ & $7461(5)$ & $6432(5)$ & $9346(2)$ & $29(2)$ \\
\hline$C(5)$ & $7804(6)$ & $5452(5)$ & $9549(2)$ & $30(2)$ \\
\hline$C(6)$ & $8983(6)$ & $5192(5)$ & $9670(3)$ & $37(2)$ \\
\hline$C(7)$ & $7047(7)$ & $5396(6)$ & $10009(2)$ & $42(2)$ \\
\hline $\mathrm{C}(8)$ & $7766(5)$ & $4780(5)$ & $9211(2)$ & $30(2)$ \\
\hline $\mathrm{C}(9)$ & $7442(6)$ & $4019(5)$ & $9280(2)$ & $36(2)$ \\
\hline
\end{tabular}




\begin{tabular}{|c|c|c|c|c|}
\hline$C(10)$ & $7662(6)$ & $3598(5)$ & $8849(3)$ & $37(2)$ \\
\hline$C(11)$ & $8103(6)$ & $4106(5)$ & $8525(2)$ & $28(2)$ \\
\hline$C(12)$ & $8423(6)$ & $4031(5)$ & $8007(2)$ & $33(2)$ \\
\hline$C(13)$ & $8475(7)$ & $3070(5)$ & $7866(3)$ & $43(2)$ \\
\hline$C(14)$ & $7546(6)$ & $4766(5)$ & $7761(3)$ & $40(2)$ \\
\hline$C(15)$ & $9534(6)$ & $4148(4)$ & $7851(2)$ & $29(2)$ \\
\hline$C(16)$ & $10407(7)$ & $3686(5)$ & $7527(3)$ & $40(2)$ \\
\hline$C(17)$ & $11231(6)$ & $4113(5)$ & $7501(3)$ & $39(2)$ \\
\hline $\mathrm{C}(18)$ & $10850(6)$ & $4802(5)$ & $7815(2)$ & $33(2)$ \\
\hline$C(19)$ & $11373(6)$ & $5436(5)$ & $7961(2)$ & $34(2)$ \\
\hline $\mathrm{C}(20)$ & $11733(6)$ & $5113(6)$ & $8443(3)$ & $45(2)$ \\
\hline $\mathrm{C}(21)$ & $12435(6)$ & $5387(6)$ & $7617(3)$ & $44(2)$ \\
\hline $\mathrm{C}(22)$ & $10578(6)$ & $6426(5)$ & $7971(2)$ & $31(2)$ \\
\hline$C(23)$ & $10748(7)$ & $7233(5)$ & $7832(3)$ & $42(2)$ \\
\hline$C(24)$ & $9729(7)$ & $7952(5)$ & $7945(3)$ & $42(2)$ \\
\hline$C(25)$ & $8959(6)$ & $7559(5)$ & $8150(2)$ & $30(2)$ \\
\hline$C(26)$ & $7741(6)$ & $7987(5)$ & $8333(2)$ & $33(2)$ \\
\hline$C(27)$ & $7431(7)$ & $9046(5)$ & $8360(3)$ & $47(2)$ \\
\hline $\mathrm{C}(28)$ & $7032(6)$ & $7806(5)$ & 7999(3) & $38(2)$ \\
\hline C(29) & $3884(5)$ & $1277(5)$ & $6186(2)$ & $28(2)$ \\
\hline$C(30)$ & $2811(6)$ & $1824(5)$ & $6303(3)$ & $38(2)$ \\
\hline$C(31)$ & $2800(6)$ & $2745(5)$ & $6298(3)$ & $39(2)$ \\
\hline$C(32)$ & $3853(5)$ & $2744(5)$ & $6175(2)$ & $28(2)$ \\
\hline$C(33)$ & $4345(6)$ & $3515(5)$ & $6140(2)$ & $32(2)$ \\
\hline$C(34)$ & $3419(6)$ & $4451(5)$ & $6108(3)$ & $41(2)$ \\
\hline$C(35)$ & $4857(6)$ & $3478(5)$ & $6582(3)$ & $39(2)$ \\
\hline$C(36)$ & $5225(6)$ & $3407(5)$ & $5715(3)$ & $32(2)$ \\
\hline$C(37)$ & $5447(6)$ & $4051(5)$ & $5401(3)$ & $35(2)$ \\
\hline$C(38)$ & $6410(6)$ & $3593(5)$ & $5085(3)$ & $34(2)$ \\
\hline$C(39)$ & $6727(6)$ & $2686(5)$ & $5222(2)$ & $30(2)$ \\
\hline$C(40)$ & $7679(6)$ & $1851(5)$ & $5009(2)$ & $29(2)$ \\
\hline $\mathrm{C}(41)$ & $7227(6)$ & $1260(5)$ & $4754(2)$ & $33(2)$ \\
\hline$C(42)$ & $8474(6)$ & $2205(6)$ & $4652(3)$ & $39(2)$ \\
\hline$C(43)$ & $8264(6)$ & $1275(5)$ & $5391(2)$ & $32(2)$ \\
\hline$C(44)$ & $9365(6)$ & $863(5)$ & $5408(3)$ & $38(2)$ \\
\hline$C(45)$ & $9467(6)$ & $321(5)$ & $5832(3)$ & $37(2)$ \\
\hline$C(46)$ & $8439(5)$ & $415(4)$ & $6055(2)$ & $29(2)$ \\
\hline$C(47)$ & $8042(6)$ & $43(5)$ & $6521(2)$ & $31(2)$ \\
\hline $\mathrm{C}(48)$ & $8996(6)$ & $-770(5)$ & $6683(3)$ & $42(2)$ \\
\hline$C(49)$ & $7652(6)$ & $801(5)$ & $6884(3)$ & $40(2)$ \\
\hline$C(50)$ & $7086(5)$ & $-289(5)$ & $6485(2)$ & $26(2)$ \\
\hline $\mathrm{C}(51)$ & $6881(6)$ & $-1072(5)$ & $6654(2)$ & $34(2)$ \\
\hline$C(52)$ & $5830(6)$ & $-1010(5)$ & $6551(2)$ & $35(2)$ \\
\hline
\end{tabular}




\begin{tabular}{|c|c|c|c|c|}
\hline$C(53)$ & $5423(6)$ & $-173(5)$ & $6321(2)$ & $29(2)$ \\
\hline$C(54)$ & $4377(6)$ & $242(5)$ & $6100(2)$ & $27(2)$ \\
\hline$C(55)$ & $4641(6)$ & $85(5)$ & $5578(2)$ & $36(2)$ \\
\hline$C(56)$ & $3523(6)$ & $-220(5)$ & $6319(3)$ & $41(2)$ \\
\hline$C(57)$ & $3871(7)$ & $8513(7)$ & $10027(3)$ & $64(3)$ \\
\hline$C(58)$ & $3721(12)$ & $9359(10)$ & $10269(5)$ & $105(4)$ \\
\hline$C(59)$ & $2560(13)$ & $9580(12)$ & $10517(6)$ & $160(8)$ \\
\hline$C(60)$ & $2000(9)$ & 9151(9) & $10272(3)$ & $91(4)$ \\
\hline$C(61)$ & $1299(10)$ & $7405(9)$ & $9003(4)$ & $86(4)$ \\
\hline$C(62)$ & $387(9)$ & $7093(8)$ & $9203(4)$ & $75(3)$ \\
\hline$C(63)$ & $-235(9)$ & $7763(10)$ & $9545(5)$ & $105(5)$ \\
\hline$C(64)$ & $562(8)$ & $8119(7)$ & $9712(3)$ & $64(3)$ \\
\hline$C(65)$ & $4798(6)$ & $7204(6)$ & $8750(3)$ & $45(2)$ \\
\hline$C(66)$ & $5093(9)$ & $6197(6)$ & $8683(4)$ & $70(3)$ \\
\hline$C(67)$ & $4532(9)$ & $5830(6)$ & $9114(4)$ & $72(3)$ \\
\hline$C(68)$ & $4013(8)$ & $6636(6)$ & $9419(3)$ & $57(3)$ \\
\hline$C(69)$ & $843(9)$ & $12469(6)$ & $8687(4)$ & $72(3)$ \\
\hline$C(70)$ & $636(9)$ & $13103(7)$ & $9053(4)$ & $74(3)$ \\
\hline $\mathrm{C}(71)$ & $1715(9)$ & $12921(6)$ & $9228(4)$ & $69(3)$ \\
\hline $\mathrm{C}(72)$ & 2301(9) & $11942(6)$ & $9137(4)$ & $69(3)$ \\
\hline $\mathrm{C}(73)$ & 1192(9) & $10756(7)$ & $7577(3)$ & $61(3)$ \\
\hline$C(74)$ & $413(10)$ & $10323(9)$ & $7436(5)$ & $97(4)$ \\
\hline$C(75)$ & $-569(10)$ & $10594(7)$ & $7787(4)$ & $72(3)$ \\
\hline$C(76)$ & $-100(7)$ & $10586(6)$ & $8221(3)$ & $57(2)$ \\
\hline$C(77)$ & $4293(6)$ & $10005(6)$ & $7737(3)$ & $47(2)$ \\
\hline $\mathrm{C}(78)$ & $5035(8)$ & $10532(7)$ & $7538(3)$ & $62(3)$ \\
\hline$C(79)$ & $4255(9)$ & $11455(7)$ & $7397(3)$ & $63(3)$ \\
\hline$C(80)$ & $3301(8)$ & $11611(6)$ & $7777(4)$ & $68(3)$ \\
\hline $\mathrm{C}(81)$ & $8086(7)$ & $3894(6)$ & $5786(3)$ & $48(2)$ \\
\hline$C(82)$ & $8111(9)$ & $3199(7)$ & $6183(4)$ & $69(3)$ \\
\hline$C(83)$ & $9241(9)$ & $2964(6)$ & $6313(3)$ & $66(3)$ \\
\hline$C(84)$ & $9482(7)$ & $3857(5)$ & $6236(3)$ & $46(2)$ \\
\hline$C(85)$ & $8291(7)$ & $7786(5)$ & $5615(3)$ & $45(2)$ \\
\hline$C(86)$ & $8004(7)$ & $8643(5)$ & $5315(3)$ & $43(2)$ \\
\hline$C(87)$ & $8161(7)$ & $8314(5)$ & $4827(3)$ & $43(2)$ \\
\hline $\mathrm{C}(88)$ & $9039(7)$ & $7383(5)$ & $4841(3)$ & $42(2)$ \\
\hline$C(89)$ & $10220(7)$ & $6018(6)$ & $6268(3)$ & $46(2)$ \\
\hline$C(90)$ & $9994(8)$ & $6442(6)$ & $6731(3)$ & $52(2)$ \\
\hline $\mathrm{C}(91)$ & $9063(7)$ & $6127(6)$ & $6984(3)$ & $48(2)$ \\
\hline$C(92)$ & $8399(6)$ & $6172(5)$ & $6606(2)$ & $38(2)$ \\
\hline$C(93)$ & $6312(7)$ & $6347(7)$ & $5948(3)$ & $63(3)$ \\
\hline$C(94)$ & $5282(7)$ & $6917(7)$ & $5770(3)$ & $61(3)$ \\
\hline$C(95)$ & $5650(6)$ & $7245(6)$ & $5287(3)$ & $45(2)$ \\
\hline
\end{tabular}




\begin{tabular}{rrrrr}
$\mathrm{C}(96)$ & $6669(6)$ & $6454(6)$ & $5136(3)$ & $41(2)$ \\
$\mathrm{C}(97)$ & $2370(11)$ & $7559(14)$ & $6646(4)$ & $148(8)$ \\
$\mathrm{C}(98)$ & $3350(9)$ & $7214(9)$ & $6888(3)$ & $69(3)$ \\
$\mathrm{C}(99)$ & $4171(13)$ & $6281(9)$ & $6838(4)$ & $88(5)$ \\
$\mathrm{C}(100)$ & $5059(11)$ & $6052(8)$ & $7080(4)$ & $83(4)$ \\
$\mathrm{C}(101)$ & $5215(10)$ & $6611(9)$ & $7334(4)$ & $78(3)$ \\
$\mathrm{C}(102)$ & $4473(9)$ & $7451(7)$ & $7385(4)$ & $66(3)$ \\
$\mathrm{C}(103)$ & $3570(8)$ & $7769(6)$ & $7176(3)$ & $52(2)$ \\
$\mathrm{C}(104)$ & $4934(13)$ & $3818(13)$ & $8291(9)$ & $123(6)$ \\
$\mathrm{C}(105)$ & $5220(50)$ & $3050(30)$ & $8068(15)$ & $150(20)$ \\
$\mathrm{C}(106)$ & $5090(20)$ & $3050(20)$ & $8568(14)$ & $96(11)$ \\
$\mathrm{C}(107)$ & $5700(30)$ & $2198(13)$ & $8421(10)$ & $57(7)$ \\
$\mathrm{C}(108)$ & $5220(40)$ & $2420(30)$ & $8866(11)$ & $270(20)$ \\
$\mathrm{C}(109)$ & $4701(17)$ & $3401(16)$ & $8911(11)$ & $173(11)$ \\
$\mathrm{C}(110)$ & $5690(50)$ & $1720(40)$ & $8550(20)$ & $190(20)$ \\
$\mathrm{C}(111)$ & $5470(20)$ & $3492(17)$ & $7751(12)$ & $81(7)$ \\
$\mathrm{C}(112)$ & $6110(20)$ & $2680(40)$ & $7641(12)$ & $139(16)$ \\
$\mathrm{C}(113)$ & $6010(30)$ & $2140(30)$ & $8089(14)$ & $232(17)$ \\
\hline
\end{tabular}

${ }^{a} \mathrm{U}_{\mathrm{eq}}$ is defined as one third of the trace of the orthogonalized $\mathrm{U}_{\mathrm{ij}}$ tensor. 
Table S10. Bond lengths (in $\AA$ ) for $\left[(\mathrm{THF})_{3} \mathrm{Fe}^{\mathrm{II}}(\mu-\mathrm{Cl})_{3} \mathrm{Fe}^{\mathrm{II}}(\mathrm{THF})_{3}\right]$ $\left[(\mathrm{THF})_{4} \mathrm{Fe}^{\mathrm{II}}(\mu-\mathrm{Cl})_{2} \mathrm{Fe}^{\mathrm{II}}(\mathrm{THF})_{4}\right]_{1 / 2}\left[\mathrm{LFe}^{\mathrm{III}}\right]_{2} \cdot 2 \mathrm{PhCH}_{3}$.

\begin{tabular}{|c|c|c|c|}
\hline Bond & Distance & Bond & Distance \\
\hline $\mathrm{Fe}(1)-\mathrm{N}(2)$ & $1.893(5)$ & $\mathrm{O}(6)-\mathrm{C}(77)$ & $1.454(9)$ \\
\hline $\mathrm{Fe}(1)-\mathrm{N}(4)$ & $1.899(5)$ & $\mathrm{O}(6)-\mathrm{C}(80)$ & $1.455(9)$ \\
\hline $\mathrm{Fe}(1)-\mathrm{N}(1)$ & $1.900(5)$ & $\mathrm{O}(7)-\mathrm{C}(81)$ & $1.470(9)$ \\
\hline $\mathrm{Fe}(1)-\mathrm{N}(3)$ & $1.913(5)$ & $\mathrm{O}(7)-\mathrm{C}(84)$ & $1.475(9)$ \\
\hline $\mathrm{Fe}(2)-\mathrm{N}(5)$ & $1.903(5)$ & $\mathrm{O}(8)-\mathrm{C}(88)$ & $1.453(9)$ \\
\hline $\mathrm{Fe}(2)-\mathrm{N}(7)$ & $1.903(5)$ & $\mathrm{O}(8)-\mathrm{C}(85)$ & $1.454(8)$ \\
\hline $\mathrm{Fe}(2)-\mathrm{N}(8)$ & $1.904(5)$ & $\mathrm{O}(9)-\mathrm{C}(89)$ & $1.442(9)$ \\
\hline $\mathrm{Fe}(2)-\mathrm{N}(6)$ & $1.907(5)$ & $\mathrm{O}(9)-\mathrm{C}(92)$ & $1.444(8)$ \\
\hline $\mathrm{Fe}(3)-\mathrm{O}(4)$ & $2.108(5)$ & $\mathrm{O}(10)-\mathrm{C}(93)$ & $1.445(9)$ \\
\hline $\mathrm{Fe}(3)-\mathrm{O}(6)$ & $2.114(5)$ & $\mathrm{O}(10)-\mathrm{C}(96)$ & $1.459(9)$ \\
\hline $\mathrm{Fe}(3)-\mathrm{O}(5)$ & $2.192(5)$ & $\mathrm{N}(1)-\mathrm{C}(1)$ & $1.392(8)$ \\
\hline $\mathrm{Fe}(3)-\mathrm{Cl}(1)$ & $2.432(2)$ & $\mathrm{N}(1)-\mathrm{C}(4)$ & $1.394(8)$ \\
\hline $\mathrm{Fe}(3)-\mathrm{Cl}(3)$ & $2.482(2)$ & $\mathrm{N}(2)-\mathrm{C}(22)$ & $1.384(9)$ \\
\hline $\mathrm{Fe}(3)-\mathrm{Cl}(2)$ & $2.498(2)$ & $\mathrm{N}(2)-\mathrm{C}(25)$ & $1.391(9)$ \\
\hline $\mathrm{Fe}(4)-\mathrm{O}(3)$ & $2.111(5)$ & $\mathrm{N}(3)-\mathrm{C}(15)$ & $1.378(9)$ \\
\hline $\mathrm{Fe}(4)-\mathrm{O}(2)$ & $2.127(5)$ & $\mathrm{N}(3)-\mathrm{C}(18)$ & $1.385(9)$ \\
\hline $\mathrm{Fe}(4)-\mathrm{O}(1)$ & $2.156(5)$ & $\mathrm{N}(4)-\mathrm{C}(11)$ & $1.380(9)$ \\
\hline $\mathrm{Fe}(4)-\mathrm{Cl}(2)$ & $2.468(2)$ & $\mathrm{N}(4)-\mathrm{C}(8)$ & $1.397(8)$ \\
\hline $\mathrm{Fe}(4)-\mathrm{Cl}(3)$ & $2.478(2)$ & $\mathrm{N}(5)-\mathrm{C}(29)$ & $1.375(9)$ \\
\hline $\mathrm{Fe}(4)-\mathrm{Cl}(1)$ & $2.518(2)$ & $\mathrm{N}(5)-\mathrm{C}(32)$ & $1.397(8)$ \\
\hline $\mathrm{Fe}(5)-\mathrm{O}(7)$ & $2.136(5)$ & $\mathrm{N}(6)-\mathrm{C}(36)$ & $1.376(9)$ \\
\hline $\mathrm{Fe}(5)-\mathrm{O}(8)$ & $2.142(5)$ & $\mathrm{N}(6)-\mathrm{C}(39)$ & $1.382(8)$ \\
\hline $\mathrm{Fe}(5)-\mathrm{O}(10)$ & $2.173(5)$ & $\mathrm{N}(7)-\mathrm{C}(43)$ & $1.379(9)$ \\
\hline $\mathrm{Fe}(5)-\mathrm{O}(9)$ & $2.203(5)$ & $\mathrm{N}(7)-\mathrm{C}(46)$ & $1.399(8)$ \\
\hline $\mathrm{Fe}(5)-\mathrm{Cl}(4)$ & $2.447(2)$ & $\mathrm{N}(8)-\mathrm{C}(50)$ & $1.380(8)$ \\
\hline $\mathrm{Fe}(5)-\mathrm{Cl}\left(4^{\prime}\right)$ & $2.490(2)$ & $\mathrm{N}(8)-\mathrm{C}(53)$ & $1.385(9)$ \\
\hline $\mathrm{Cl}(4)-\mathrm{Fe}\left(5^{\prime}\right)$ & $2.490(2)$ & $C(1)-C(2)$ & $1.366(10)$ \\
\hline $\mathrm{O}(1)-\mathrm{C}(57)$ & $1.441(10)$ & $C(1)-C(26)$ & $1.499(10)$ \\
\hline $\mathrm{O}(1)-\mathrm{C}(60)$ & $1.444(10)$ & $C(2)-C(3)$ & $1.412(10)$ \\
\hline $\mathrm{O}(2)-\mathrm{C}(64)$ & $1.436(9)$ & $C(3)-C(4)$ & $1.368(10)$ \\
\hline $\mathrm{O}(2)-\mathrm{C}(61)$ & $1.440(10)$ & $C(4)-C(5)$ & $1.512(10)$ \\
\hline $\mathrm{O}(3)-\mathrm{C}(68)$ & $1.431(9)$ & $C(5)-C(8)$ & $1.509(10)$ \\
\hline $\mathrm{O}(3)-\mathrm{C}(65)$ & $1.445(8)$ & $C(5)-C(7)$ & $1.537(9)$ \\
\hline $\mathrm{O}(4)-\mathrm{C}(69)$ & $1.447(10)$ & $C(5)-C(6)$ & $1.541(10)$ \\
\hline $\mathrm{O}(4)-\mathrm{C}(72)$ & $1.474(10)$ & $\mathrm{C}(8)-\mathrm{C}(9)$ & $1.362(10)$ \\
\hline $\mathrm{O}(5)-\mathrm{C}(73)$ & $1.450(10)$ & $\mathrm{C}(9)-\mathrm{C}(10)$ & $1.409(11)$ \\
\hline $\mathrm{O}(5)-\mathrm{C}(76)$ & $1.457(10)$ & $\mathrm{C}(10)-\mathrm{C}(11)$ & $1.350(10)$ \\
\hline
\end{tabular}




\begin{tabular}{|c|c|c|c|}
\hline$C(11)-C(12)$ & $1.501(10)$ & $C(54)-C(55)$ & $1.526(9)$ \\
\hline$C(12)-C(15)$ & $1.507(10)$ & $\mathrm{C}(54)-\mathrm{C}(56)$ & $1.538(10)$ \\
\hline $\mathrm{C}(12)-\mathrm{C}(14)$ & $1.528(10)$ & $\mathrm{C}(57)-\mathrm{C}(58)$ & $1.476(15)$ \\
\hline$C(12)-C(13)$ & $1.545(10)$ & $\mathrm{C}(58)-\mathrm{C}(59)$ & $1.497(19)$ \\
\hline$C(15)-C(16)$ & $1.374(10)$ & $C(59)-C(60)$ & $1.443(17)$ \\
\hline$C(16)-C(17)$ & $1.427(11)$ & $\mathrm{C}(61)-\mathrm{C}(62)$ & $1.444(13)$ \\
\hline $\mathrm{C}(17)-\mathrm{C}(18)$ & $1.367(10)$ & $C(62)-C(63)$ & $1.431(15)$ \\
\hline $\mathrm{C}(18)-\mathrm{C}(19)$ & $1.493(11)$ & $\mathrm{C}(63)-\mathrm{C}(64)$ & $1.493(14)$ \\
\hline $\mathrm{C}(19)-\mathrm{C}(22)$ & $1.518(10)$ & $C(65)-C(66)$ & $1.485(12)$ \\
\hline$C(19)-C(20)$ & $1.545(10)$ & $\mathrm{C}(66)-\mathrm{C}(67)$ & $1.505(13)$ \\
\hline $\mathrm{C}(19)-\mathrm{C}(21)$ & $1.545(10)$ & $C(67)-C(68)$ & $1.486(13)$ \\
\hline $\mathrm{C}(22)-\mathrm{C}(23)$ & $1.349(10)$ & $C(69)-C(70)$ & $1.440(13)$ \\
\hline $\mathrm{C}(23)-\mathrm{C}(24)$ & $1.414(11)$ & $\mathrm{C}(70)-\mathrm{C}(71)$ & $1.503(14)$ \\
\hline $\mathrm{C}(24)-\mathrm{C}(25)$ & $1.369(10)$ & $\mathrm{C}(71)-\mathrm{C}(72)$ & $1.460(12)$ \\
\hline$C(25)-C(26)$ & $1.512(10)$ & $\mathrm{C}(73)-\mathrm{C}(74)$ & $1.515(14)$ \\
\hline$C(26)-C(27)$ & $1.543(10)$ & $C(74)-C(75)$ & $1.455(15)$ \\
\hline $\mathrm{C}(26)-\mathrm{C}(28)$ & $1.553(10)$ & $C(75)-C(76)$ & $1.495(13)$ \\
\hline $\mathrm{C}(29)-\mathrm{C}(30)$ & $1.361(10)$ & $\mathrm{C}(77)-\mathrm{C}(78)$ & $1.479(12)$ \\
\hline $\mathrm{C}(29)-\mathrm{C}(54)$ & $1.524(9)$ & $\mathrm{C}(78)-\mathrm{C}(79)$ & $1.501(14)$ \\
\hline $\mathrm{C}(30)-\mathrm{C}(31)$ & $1.410(11)$ & $C(79)-C(80)$ & $1.483(12)$ \\
\hline $\mathrm{C}(31)-\mathrm{C}(32)$ & $1.355(10)$ & $\mathrm{C}(81)-\mathrm{C}(82)$ & $1.498(13)$ \\
\hline $\mathrm{C}(32)-\mathrm{C}(33)$ & $1.519(10)$ & $\mathrm{C}(82)-\mathrm{C}(83)$ & $1.495(14)$ \\
\hline$C(33)-C(36)$ & $1.516(10)$ & $\mathrm{C}(83)-\mathrm{C}(84)$ & $1.500(12)$ \\
\hline $\mathrm{C}(33)-\mathrm{C}(34)$ & $1.534(9)$ & $C(85)-C(86)$ & $1.484(11)$ \\
\hline $\mathrm{C}(33)-\mathrm{C}(35)$ & $1.538(10)$ & $\mathrm{C}(86)-\mathrm{C}(87)$ & $1.513(11)$ \\
\hline$C(36)-C(37)$ & $1.357(10)$ & $\mathrm{C}(87)-\mathrm{C}(88)$ & $1.496(11)$ \\
\hline $\mathrm{C}(37)-\mathrm{C}(38)$ & $1.425(10)$ & $\mathrm{C}(89)-\mathrm{C}(90)$ & $1.493(11)$ \\
\hline $\mathrm{C}(38)-\mathrm{C}(39)$ & $1.353(10)$ & $\mathrm{C}(90)-\mathrm{C}(91)$ & $1.510(12)$ \\
\hline$C(39)-C(40)$ & $1.535(10)$ & $\mathrm{C}(91)-\mathrm{C}(92)$ & $1.497(11)$ \\
\hline $\mathrm{C}(40)-\mathrm{C}(43)$ & $1.498(10)$ & $\mathrm{C}(93)-\mathrm{C}(94)$ & $1.476(12)$ \\
\hline $\mathrm{C}(40)-\mathrm{C}(41)$ & $1.539(10)$ & $\mathrm{C}(94)-\mathrm{C}(95)$ & $1.506(12)$ \\
\hline$C(40)-C(42)$ & $1.539(9)$ & $C(95)-C(96)$ & $1.491(10)$ \\
\hline$C(43)-C(44)$ & $1.360(10)$ & $\mathrm{C}(97)-\mathrm{C}(98)$ & $1.477(16)$ \\
\hline$C(44)-C(45)$ & $1.424(10)$ & $\mathrm{C}(98)-\mathrm{C}(103)$ & $1.378(13)$ \\
\hline$C(45)-C(46)$ & $1.349(10)$ & C(98)-C(99) & $1.467(17)$ \\
\hline$C(46)-C(47)$ & $1.504(10)$ & C(99)-C(100) & $1.382(18)$ \\
\hline$C(47)-C(50)$ & $1.519(10)$ & $C(100)-C(101)$ & $1.273(17)$ \\
\hline $\mathrm{C}(47)-\mathrm{C}(48)$ & $1.533(10)$ & $C(101)-C(102)$ & $1.323(14)$ \\
\hline $\mathrm{C}(47)-\mathrm{C}(49)$ & $1.542(10)$ & $C(102)-C(103)$ & $1.336(13)$ \\
\hline $\mathrm{C}(50)-\mathrm{C}(51)$ & $1.353(10)$ & C(104)-C(105) & $1.30(5)$ \\
\hline $\mathrm{C}(51)-\mathrm{C}(52)$ & $1.419(10)$ & $C(104)-C(106)$ & $1.35(4)$ \\
\hline $\mathrm{C}(52)-\mathrm{C}(53)$ & $1.373(10)$ & C(104)-C(111) & $1.66(4)$ \\
\hline C(53)-C(54) & $1.516(10)$ & C(104)-C(109) & $1.87(4)$ \\
\hline
\end{tabular}




\begin{tabular}{llll}
$\mathrm{C}(105)-\mathrm{C}(111)$ & $1.16(4)$ & $\mathrm{C}(107)-\mathrm{C}(110)$ & $0.79(7)$ \\
$\mathrm{C}(105)-\mathrm{C}(113)$ & $1.43(6)$ & $\mathrm{C}(107)-\mathrm{C}(113)$ & $0.98(5)$ \\
$\mathrm{C}(105)-\mathrm{C}(106)$ & $1.44(6)$ & $\mathrm{C}(107)-\mathrm{C}(108)$ & $1.36(5)$ \\
$\mathrm{C}(105)-\mathrm{C}(112)$ & $1.55(6)$ & $\mathrm{C}(108)-\mathrm{C}(110)$ & $1.39(6)$ \\
$\mathrm{C}(105)-\mathrm{C}(107)$ & $1.61(4)$ & $\mathrm{C}(108)-\mathrm{C}(109)$ & $1.43(4)$ \\
$\mathrm{C}(106)-\mathrm{C}(109)$ & $1.14(4)$ & $\mathrm{C}(110)-\mathrm{C}(113)$ & $1.49(7)$ \\
$\mathrm{C}(106)-\mathrm{C}(108)$ & $1.22(4)$ & $\mathrm{C}(111)-\mathrm{C}(112)$ & $1.28(4)$ \\
$\mathrm{C}(106)-\mathrm{C}(107)$ & $1.34(4)$ & $\mathrm{C}(112)-\mathrm{C}(113)$ & $1.49(6)$ \\
\hline
\end{tabular}


Table S11. Bond angles (in deg) for $\left[(\mathrm{THF})_{3} \mathrm{Fe}^{\mathrm{II}}(\mu-\mathrm{Cl})_{3} \mathrm{Fe}^{\mathrm{II}}(\mathrm{THF})_{3}\right]\left[(\mathrm{THF})_{4} \mathrm{Fe}^{\mathrm{II}}(\mu-\mathrm{Cl})_{2}\right.$ $\left.\mathrm{Fe}^{\mathrm{II}}(\mathrm{THF})_{4}\right]_{1 / 2}\left[\mathrm{LFe}^{\mathrm{III}}\right]_{2} \bullet 2 \mathrm{PhCH}_{3}$.

\begin{tabular}{|c|c|c|c|}
\hline Bond angle & Angle & Bond angle & Angle \\
\hline $\mathrm{N}(2)-\mathrm{Fe}(1)-\mathrm{N}(4)$ & $179.1(2)$ & $\mathrm{Cl}(3)-\mathrm{Fe}(3)-\mathrm{Cl}(2)$ & $86.77(7)$ \\
\hline $\mathrm{N}(2)-\mathrm{Fe}(1)-\mathrm{N}(1)$ & $90.6(2)$ & $\mathrm{O}(3)-\mathrm{Fe}(4)-\mathrm{O}(2)$ & $86.0(2)$ \\
\hline $\mathrm{N}(4)-\mathrm{Fe}(1)-\mathrm{N}(1)$ & $90.1(2)$ & $\mathrm{O}(3)-\mathrm{Fe}(4)-\mathrm{O}(1)$ & $86.4(2)$ \\
\hline $\mathrm{N}(2)-\mathrm{Fe}(1)-\mathrm{N}(3)$ & $89.5(2)$ & $\mathrm{O}(2)-\mathrm{Fe}(4)-\mathrm{O}(1)$ & $91.3(2)$ \\
\hline $\mathrm{N}(4)-\mathrm{Fe}(1)-\mathrm{N}(3)$ & $89.9(2)$ & $\mathrm{O}(3)-\mathrm{Fe}(4)-\mathrm{Cl}(2)$ & $96.07(15)$ \\
\hline $\mathrm{N}(1)-\mathrm{Fe}(1)-\mathrm{N}(3)$ & $179.3(2)$ & $\mathrm{O}(2)-\mathrm{Fe}(4)-\mathrm{Cl}(2)$ & $177.10(16)$ \\
\hline $\mathrm{N}(5)-\mathrm{Fe}(2)-\mathrm{N}(7)$ & $179.5(3)$ & $\mathrm{O}(1)-\mathrm{Fe}(4)-\mathrm{Cl}(2)$ & $90.88(16)$ \\
\hline $\mathrm{N}(5)-\mathrm{Fe}(2)-\mathrm{N}(8)$ & $90.1(2)$ & $\mathrm{O}(3)-\mathrm{Fe}(4)-\mathrm{Cl}(3)$ & $175.60(17)$ \\
\hline $\mathrm{N}(7)-\mathrm{Fe}(2)-\mathrm{N}(8)$ & $90.0(2)$ & $\mathrm{O}(2)-\mathrm{Fe}(4)-\mathrm{Cl}(3)$ & $90.33(15)$ \\
\hline $\mathrm{N}(5)-\mathrm{Fe}(2)-\mathrm{N}(6)$ & $89.8(2)$ & $\mathrm{O}(1)-\mathrm{Fe}(4)-\mathrm{Cl}(3)$ & $96.13(15)$ \\
\hline $\mathrm{N}(7)-\mathrm{Fe}(2)-\mathrm{N}(6)$ & $90.1(2)$ & $\mathrm{Cl}(2)-\mathrm{Fe}(4)-\mathrm{Cl}(3)$ & $87.54(7)$ \\
\hline $\mathrm{N}(8)-\mathrm{Fe}(2)-\mathrm{N}(6)$ & $179.3(2)$ & $\mathrm{O}(3)-\mathrm{Fe}(4)-\mathrm{Cl}(1)$ & $91.11(15)$ \\
\hline $\mathrm{O}(4)-\mathrm{Fe}(3)-\mathrm{O}(6)$ & $86.4(2)$ & $\mathrm{O}(2)-\mathrm{Fe}(4)-\mathrm{Cl}(1)$ & $92.84(15)$ \\
\hline $\mathrm{O}(4)-\mathrm{Fe}(3)-\mathrm{O}(5)$ & $94.1(2)$ & $\mathrm{O}(1)-\mathrm{Fe}(4)-\mathrm{Cl}(1)$ & $174.99(16)$ \\
\hline $\mathrm{O}(6)-\mathrm{Fe}(3)-\mathrm{O}(5)$ & $88.3(2)$ & $\mathrm{Cl}(2)-\mathrm{Fe}(4)-\mathrm{Cl}(1)$ & $85.08(7)$ \\
\hline $\mathrm{O}(4)-\mathrm{Fe}(3)-\mathrm{Cl}(1)$ & $176.15(17)$ & $\mathrm{Cl}(3)-\mathrm{Fe}(4)-\mathrm{Cl}(1)$ & $86.67(7)$ \\
\hline $\mathrm{O}(6)-\mathrm{Fe}(3)-\mathrm{Cl}(1)$ & $97.48(15)$ & $\mathrm{O}(7)-\mathrm{Fe}(5)-\mathrm{O}(8)$ & $161.67(19)$ \\
\hline $\mathrm{O}(5)-\mathrm{Fe}(3)-\mathrm{Cl}(1)$ & $85.80(14)$ & $\mathrm{O}(7)-\mathrm{Fe}(5)-\mathrm{O}(10)$ & $87.4(2)$ \\
\hline $\mathrm{O}(4)-\mathrm{Fe}(3)-\mathrm{Cl}(3)$ & $87.68(16)$ & $\mathrm{O}(8)-\mathrm{Fe}(5)-\mathrm{O}(10)$ & $83.18(19)$ \\
\hline $\mathrm{O}(6)-\mathrm{Fe}(3)-\mathrm{Cl}(3)$ & $173.48(16)$ & $\mathrm{O}(7)-\mathrm{Fe}(5)-\mathrm{O}(9)$ & $82.70(18)$ \\
\hline $\mathrm{O}(5)-\mathrm{Fe}(3)-\mathrm{Cl}(3)$ & $94.78(15)$ & $\mathrm{O}(8)-\mathrm{Fe}(5)-\mathrm{O}(9)$ & $83.53(18)$ \\
\hline $\mathrm{Cl}(1)-\mathrm{Fe}(3)-\mathrm{Cl}(3)$ & $88.49(7)$ & $\mathrm{O}(10)-\mathrm{Fe}(5)-\mathrm{O}(9)$ & $99.98(18)$ \\
\hline $\mathrm{O}(4)-\mathrm{Fe}(3)-\mathrm{Cl}(2)$ & $93.88(15)$ & $\mathrm{O}(7)-\mathrm{Fe}(5)-\mathrm{Cl}(4)$ & $98.13(15)$ \\
\hline $\mathrm{O}(6)-\mathrm{Fe}(3)-\mathrm{Cl}(2)$ & $90.99(16)$ & $\mathrm{O}(8)-\mathrm{Fe}(5)-\mathrm{Cl}(4)$ & $94.14(14)$ \\
\hline $\mathrm{O}(5)-\mathrm{Fe}(3)-\mathrm{Cl}(2)$ & $171.88(15)$ & $\mathrm{O}(10)-\mathrm{Fe}(5)-\mathrm{Cl}(4)$ & $168.35(14)$ \\
\hline $\mathrm{Cl}(1)-\mathrm{Fe}(3)-\mathrm{Cl}(2)$ & $86.27(7)$ & $\mathrm{O}(9)-\mathrm{Fe}(5)-\mathrm{Cl}(4)$ & $90.95(13)$ \\
\hline $\mathrm{O}(7)-\mathrm{Fe}(5)-\mathrm{Cl}\left(4^{\prime}\right)$ & $93.82(15)$ & $\mathrm{C}(22)-\mathrm{N}(2)-\mathrm{Fe}(1)$ & $127.0(5)$ \\
\hline $\mathrm{O}(8)-\mathrm{Fe}(5)-\mathrm{Cl}\left(4^{\prime}\right)$ & $101.38(14)$ & $\mathrm{C}(25)-\mathrm{N}(2)-\mathrm{Fe}(1)$ & $125.4(5)$ \\
\hline $\mathrm{O}(10)-\mathrm{Fe}(5)-\mathrm{Cl}\left(4^{\prime}\right)$ & $87.47(14)$ & $\mathrm{C}(15)-\mathrm{N}(3)-\mathrm{C}(18)$ & $108.9(6)$ \\
\hline $\mathrm{O}(9)-\mathrm{Fe}(5)-\mathrm{Cl}\left(4^{\prime}\right)$ & $171.59(14)$ & $\mathrm{C}(15)-\mathrm{N}(3)-\mathrm{Fe}(1)$ & $124.8(4)$ \\
\hline $\mathrm{Cl}(4)-\mathrm{Fe}(5)-\mathrm{Cl}\left(4^{\prime}\right)$ & $81.93(7)$ & $\mathrm{C}(18)-\mathrm{N}(3)-\mathrm{Fe}(1)$ & $126.0(5)$ \\
\hline $\mathrm{Fe}(3)-\mathrm{Cl}(1)-\mathrm{Fe}(4)$ & $75.12(6)$ & $\mathrm{C}(11)-\mathrm{N}(4)-\mathrm{C}(8)$ & $108.1(5)$ \\
\hline $\mathrm{Fe}(4)-\mathrm{Cl}(2)-\mathrm{Fe}(3)$ & $74.86(6)$ & $\mathrm{C}(11)-\mathrm{N}(4)-\mathrm{Fe}(1)$ & $126.3(4)$ \\
\hline $\mathrm{Fe}(4)-\mathrm{Cl}(3)-\mathrm{Fe}(3)$ & $74.96(6)$ & $\mathrm{C}(8)-\mathrm{N}(4)-\mathrm{Fe}(1)$ & $125.5(5)$ \\
\hline $\mathrm{Fe}(5)-\mathrm{Cl}(4)-\mathrm{Fe}\left(5^{\prime}\right)$ & $98.07(7)$ & $\mathrm{C}(29)-\mathrm{N}(5)-\mathrm{C}(32)$ & $107.8(5)$ \\
\hline $\mathrm{C}(57)-\mathrm{O}(1)-\mathrm{C}(60)$ & $105.8(7)$ & $\mathrm{C}(29)-\mathrm{N}(5)-\mathrm{Fe}(2)$ & $126.2(4)$ \\
\hline $\mathrm{C}(57)-\mathrm{O}(1)-\mathrm{Fe}(4)$ & $121.4(5)$ & $\mathrm{C}(32)-\mathrm{N}(5)-\mathrm{Fe}(2)$ & $125.9(5)$ \\
\hline $\mathrm{C}(60)-\mathrm{O}(1)-\mathrm{Fe}(4)$ & $125.2(5)$ & $\mathrm{C}(36)-\mathrm{N}(6)-\mathrm{C}(39)$ & $107.7(6)$ \\
\hline
\end{tabular}




\begin{tabular}{|c|c|c|c|}
\hline $\mathrm{C}(64)-\mathrm{O}(2)-\mathrm{C}(61)$ & $108.2(6)$ & $\mathrm{C}(36)-\mathrm{N}(6)-\mathrm{Fe}(2)$ & $126.1(5)$ \\
\hline $\mathrm{C}(64)-\mathrm{O}(2)-\mathrm{Fe}(4)$ & $125.4(5)$ & $\mathrm{C}(39)-\mathrm{N}(6)-\mathrm{Fe}(2)$ & $126.1(4)$ \\
\hline $\mathrm{C}(61)-\mathrm{O}(2)-\mathrm{Fe}(4)$ & $123.3(5)$ & $\mathrm{C}(43)-\mathrm{N}(7)-\mathrm{C}(46)$ & $107.6(5)$ \\
\hline $\mathrm{C}(68)-\mathrm{O}(3)-\mathrm{C}(65)$ & $105.4(6)$ & $\mathrm{C}(43)-\mathrm{N}(7)-\mathrm{Fe}(2)$ & $126.2(5)$ \\
\hline $\mathrm{C}(68)-\mathrm{O}(3)-\mathrm{Fe}(4)$ & $128.5(4)$ & $\mathrm{C}(46)-\mathrm{N}(7)-\mathrm{Fe}(2)$ & $126.1(4)$ \\
\hline $\mathrm{C}(65)-\mathrm{O}(3)-\mathrm{Fe}(4)$ & $126.0(4)$ & $\mathrm{C}(50)-\mathrm{N}(8)-\mathrm{C}(53)$ & $108.3(5)$ \\
\hline $\mathrm{C}(69)-\mathrm{O}(4)-\mathrm{C}(72)$ & $107.9(6)$ & $\mathrm{C}(50)-\mathrm{N}(8)-\mathrm{Fe}(2)$ & $126.0(5)$ \\
\hline $\mathrm{C}(69)-\mathrm{O}(4)-\mathrm{Fe}(3)$ & $120.0(5)$ & $\mathrm{C}(53)-\mathrm{N}(8)-\mathrm{Fe}(2)$ & $125.7(4)$ \\
\hline $\mathrm{C}(72)-\mathrm{O}(4)-\mathrm{Fe}(3)$ & $122.6(5)$ & $\mathrm{C}(2)-\mathrm{C}(1)-\mathrm{N}(1)$ & $107.9(6)$ \\
\hline $\mathrm{C}(73)-\mathrm{O}(5)-\mathrm{C}(76)$ & $108.4(7)$ & $C(2)-C(1)-C(26)$ & $131.6(6)$ \\
\hline $\mathrm{C}(73)-\mathrm{O}(5)-\mathrm{Fe}(3)$ & $119.5(5)$ & $\mathrm{N}(1)-\mathrm{C}(1)-\mathrm{C}(26)$ & $120.3(6)$ \\
\hline $\mathrm{C}(76)-\mathrm{O}(5)-\mathrm{Fe}(3)$ & $119.4(5)$ & $\mathrm{C}(1)-\mathrm{C}(2)-\mathrm{C}(3)$ & $108.3(6)$ \\
\hline $\mathrm{C}(77)-\mathrm{O}(6)-\mathrm{C}(80)$ & $108.7(6)$ & $C(4)-C(3)-C(2)$ & $107.5(6)$ \\
\hline $\mathrm{C}(77)-\mathrm{O}(6)-\mathrm{Fe}(3)$ & $123.4(4)$ & $\mathrm{C}(3)-\mathrm{C}(4)-\mathrm{N}(1)$ & $108.3(6)$ \\
\hline $\mathrm{C}(80)-\mathrm{O}(6)-\mathrm{Fe}(3)$ & $126.3(5)$ & $C(3)-C(4)-C(5)$ & $131.0(6)$ \\
\hline $\mathrm{C}(81)-\mathrm{O}(7)-\mathrm{C}(84)$ & $109.0(6)$ & $\mathrm{N}(1)-\mathrm{C}(4)-\mathrm{C}(5)$ & $120.4(6)$ \\
\hline $\mathrm{C}(81)-\mathrm{O}(7)-\mathrm{Fe}(5)$ & $124.6(5)$ & $C(8)-C(5)-C(4)$ & $111.1(6)$ \\
\hline $\mathrm{C}(84)-\mathrm{O}(7)-\mathrm{Fe}(5)$ & $125.8(4)$ & $C(8)-C(5)-C(7)$ & $110.0(6)$ \\
\hline $\mathrm{C}(88)-\mathrm{O}(8)-\mathrm{C}(85)$ & $107.9(5)$ & $C(4)-C(5)-C(7)$ & $110.0(6)$ \\
\hline $\mathrm{C}(88)-\mathrm{O}(8)-\mathrm{Fe}(5)$ & $125.9(4)$ & $C(8)-C(5)-C(6)$ & $110.4(6)$ \\
\hline $\mathrm{C}(85)-\mathrm{O}(8)-\mathrm{Fe}(5)$ & $124.8(4)$ & $C(4)-C(5)-C(6)$ & $108.8(6)$ \\
\hline $\mathrm{C}(89)-\mathrm{O}(9)-\mathrm{C}(92)$ & $108.7(5)$ & $C(7)-C(5)-C(6)$ & $106.4(6)$ \\
\hline $\mathrm{C}(89)-\mathrm{O}(9)-\mathrm{Fe}(5)$ & $121.6(4)$ & $\mathrm{C}(9)-\mathrm{C}(8)-\mathrm{N}(4)$ & $107.5(6)$ \\
\hline $\mathrm{C}(92)-\mathrm{O}(9)-\mathrm{Fe}(5)$ & $129.6(4)$ & $C(9)-C(8)-C(5)$ & $131.4(6)$ \\
\hline $\mathrm{C}(93)-\mathrm{O}(10)-\mathrm{C}(96)$ & $107.1(5)$ & $\mathrm{N}(4)-\mathrm{C}(8)-\mathrm{C}(5)$ & $120.9(6)$ \\
\hline $\mathrm{C}(93)-\mathrm{O}(10)-\mathrm{Fe}(5)$ & $132.8(5)$ & $C(8)-C(9)-C(10)$ & $107.9(6)$ \\
\hline $\mathrm{C}(96)-\mathrm{O}(10)-\mathrm{Fe}(5)$ & $119.9(4)$ & $C(11)-C(10)-C(9)$ & $108.2(6)$ \\
\hline $\mathrm{C}(1)-\mathrm{N}(1)-\mathrm{C}(4)$ & $107.9(5)$ & $C(10)-C(11)-N(4)$ & $108.4(6)$ \\
\hline $\mathrm{C}(1)-\mathrm{N}(1)-\mathrm{Fe}(1)$ & $125.9(4)$ & $C(10)-C(11)-C(12)$ & $131.9(7)$ \\
\hline $\mathrm{C}(4)-\mathrm{N}(1)-\mathrm{Fe}(1)$ & $126.1(4)$ & $\mathrm{N}(4)-\mathrm{C}(11)-\mathrm{C}(12)$ & $119.6(6)$ \\
\hline $\mathrm{C}(22)-\mathrm{N}(2)-\mathrm{C}(25)$ & $107.6(6)$ & $\mathrm{C}(11)-\mathrm{C}(12)-\mathrm{C}(15)$ & $111.0(6)$ \\
\hline $\mathrm{C}(11)-\mathrm{C}(12)-\mathrm{C}(14)$ & $109.8(6)$ & $C(32)-C(33)-C(34)$ & $109.0(6)$ \\
\hline $\mathrm{C}(15)-\mathrm{C}(12)-\mathrm{C}(14)$ & $109.7(6)$ & $\mathrm{C}(36)-\mathrm{C}(33)-\mathrm{C}(35)$ & $109.9(6)$ \\
\hline $\mathrm{C}(11)-\mathrm{C}(12)-\mathrm{C}(13)$ & $109.4(6)$ & $\mathrm{C}(32)-\mathrm{C}(33)-\mathrm{C}(35)$ & $108.9(6)$ \\
\hline $\mathrm{C}(15)-\mathrm{C}(12)-\mathrm{C}(13)$ & 109.2(6) & $\mathrm{C}(34)-\mathrm{C}(33)-\mathrm{C}(35)$ & $108.7(6)$ \\
\hline $\mathrm{C}(14)-\mathrm{C}(12)-\mathrm{C}(13)$ & $107.7(6)$ & $\mathrm{C}(37)-\mathrm{C}(36)-\mathrm{N}(6)$ & $108.4(6)$ \\
\hline$C(16)-C(15)-N(3)$ & $108.2(6)$ & $\mathrm{C}(37)-\mathrm{C}(36)-\mathrm{C}(33)$ & $130.5(7)$ \\
\hline $\mathrm{C}(16)-\mathrm{C}(15)-\mathrm{C}(12)$ & $130.8(7)$ & $\mathrm{N}(6)-\mathrm{C}(36)-\mathrm{C}(33)$ & $121.0(6)$ \\
\hline $\mathrm{N}(3)-\mathrm{C}(15)-\mathrm{C}(12)$ & $121.0(6)$ & $\mathrm{C}(36)-\mathrm{C}(37)-\mathrm{C}(38)$ & $108.0(6)$ \\
\hline$C(15)-C(16)-C(17)$ & $107.1(7)$ & $\mathrm{C}(39)-\mathrm{C}(38)-\mathrm{C}(37)$ & $106.4(6)$ \\
\hline $\mathrm{C}(18)-\mathrm{C}(17)-\mathrm{C}(16)$ & $107.8(7)$ & $\mathrm{C}(38)-\mathrm{C}(39)-\mathrm{N}(6)$ & $109.4(6)$ \\
\hline $\mathrm{C}(17)-\mathrm{C}(18)-\mathrm{N}(3)$ & $107.9(7)$ & $\mathrm{C}(38)-\mathrm{C}(39)-\mathrm{C}(40)$ & $130.7(6)$ \\
\hline $\mathrm{C}(17)-\mathrm{C}(18)-\mathrm{C}(19)$ & $131.9(7)$ & $\mathrm{N}(6)-\mathrm{C}(39)-\mathrm{C}(40)$ & $119.8(6)$ \\
\hline
\end{tabular}




\begin{tabular}{|c|c|c|c|}
\hline $\mathrm{N}(3)-\mathrm{C}(18)-\mathrm{C}(19)$ & $120.2(6)$ & $\mathrm{C}(43)-\mathrm{C}(40)-\mathrm{C}(39)$ & $109.1(6)$ \\
\hline $\mathrm{C}(18)-\mathrm{C}(19)-\mathrm{C}(22)$ & $110.5(6)$ & $\mathrm{C}(43)-\mathrm{C}(40)-\mathrm{C}(41)$ & $109.6(6)$ \\
\hline $\mathrm{C}(18)-\mathrm{C}(19)-\mathrm{C}(20)$ & $109.8(6)$ & $\mathrm{C}(39)-\mathrm{C}(40)-\mathrm{C}(41)$ & $110.3(6)$ \\
\hline$C(22)-C(19)-C(20)$ & $110.6(6)$ & $C(43)-C(40)-C(42)$ & $110.9(6)$ \\
\hline $\mathrm{C}(18)-\mathrm{C}(19)-\mathrm{C}(21)$ & $109.6(6)$ & $C(39)-C(40)-C(42)$ & $108.8(6)$ \\
\hline$C(22)-C(19)-C(21)$ & $109.5(6)$ & $\mathrm{C}(41)-\mathrm{C}(40)-\mathrm{C}(42)$ & $108.2(6)$ \\
\hline$C(20)-C(19)-C(21)$ & $106.8(6)$ & $\mathrm{C}(44)-\mathrm{C}(43)-\mathrm{N}(7)$ & $109.0(6)$ \\
\hline $\mathrm{C}(23)-\mathrm{C}(22)-\mathrm{N}(2)$ & $109.5(6)$ & $\mathrm{C}(44)-\mathrm{C}(43)-\mathrm{C}(40)$ & $130.2(6)$ \\
\hline $\mathrm{C}(23)-\mathrm{C}(22)-\mathrm{C}(19)$ & $130.9(7)$ & $N(7)-C(43)-C(40)$ & $120.5(6)$ \\
\hline $\mathrm{N}(2)-\mathrm{C}(22)-\mathrm{C}(19)$ & $119.6(6)$ & $C(43)-C(44)-C(45)$ & $107.1(6)$ \\
\hline $\mathrm{C}(22)-\mathrm{C}(23)-\mathrm{C}(24)$ & $107.2(7)$ & $C(46)-C(45)-C(44)$ & $108.0(6)$ \\
\hline$C(25)-C(24)-C(23)$ & $108.1(7)$ & $\mathrm{C}(45)-\mathrm{C}(46)-\mathrm{N}(7)$ & $108.3(6)$ \\
\hline $\mathrm{C}(24)-\mathrm{C}(25)-\mathrm{N}(2)$ & $107.7(6)$ & $C(45)-C(46)-C(47)$ & $131.5(6)$ \\
\hline$C(24)-C(25)-C(26)$ & $131.3(6)$ & $N(7)-C(46)-C(47)$ & $120.1(6)$ \\
\hline $\mathrm{N}(2)-\mathrm{C}(25)-\mathrm{C}(26)$ & $121.1(6)$ & $C(46)-C(47)-C(50)$ & $110.3(6)$ \\
\hline$C(1)-C(26)-C(25)$ & $109.3(6)$ & $\mathrm{C}(46)-\mathrm{C}(47)-\mathrm{C}(48)$ & $109.3(6)$ \\
\hline$C(1)-C(26)-C(27)$ & $109.6(6)$ & $\mathrm{C}(50)-\mathrm{C}(47)-\mathrm{C}(48)$ & $109.3(6)$ \\
\hline$C(25)-C(26)-C(27)$ & $110.1(6)$ & $C(46)-C(47)-C(49)$ & $110.3(6)$ \\
\hline$C(1)-C(26)-C(28)$ & $109.9(6)$ & $\mathrm{C}(50)-\mathrm{C}(47)-\mathrm{C}(49)$ & $108.9(6)$ \\
\hline$C(25)-C(26)-C(28)$ & $110.2(6)$ & $\mathrm{C}(48)-\mathrm{C}(47)-\mathrm{C}(49)$ & $108.7(6)$ \\
\hline$C(27)-C(26)-C(28)$ & $107.7(6)$ & $\mathrm{C}(51)-\mathrm{C}(50)-\mathrm{N}(8)$ & $108.5(6)$ \\
\hline $\mathrm{C}(30)-\mathrm{C}(29)-\mathrm{N}(5)$ & $108.8(6)$ & $\mathrm{C}(51)-\mathrm{C}(50)-\mathrm{C}(47)$ & $130.9(6)$ \\
\hline$C(30)-C(29)-C(54)$ & $130.3(7)$ & $\mathrm{N}(8)-\mathrm{C}(50)-\mathrm{C}(47)$ & $120.5(6)$ \\
\hline $\mathrm{N}(5)-\mathrm{C}(29)-\mathrm{C}(54)$ & $120.6(6)$ & $\mathrm{C}(50)-\mathrm{C}(51)-\mathrm{C}(52)$ & $108.1(6)$ \\
\hline$C(29)-C(30)-C(31)$ & $107.3(6)$ & $\mathrm{C}(53)-\mathrm{C}(52)-\mathrm{C}(51)$ & $107.0(6)$ \\
\hline$C(32)-C(31)-C(30)$ & $108.3(6)$ & $\mathrm{C}(52)-\mathrm{C}(53)-\mathrm{N}(8)$ & $108.1(6)$ \\
\hline $\mathrm{C}(31)-\mathrm{C}(32)-\mathrm{N}(5)$ & $107.8(6)$ & $\mathrm{C}(52)-\mathrm{C}(53)-\mathrm{C}(54)$ & $130.6(6)$ \\
\hline$C(31)-C(32)-C(33)$ & $131.7(6)$ & $\mathrm{N}(8)-\mathrm{C}(53)-\mathrm{C}(54)$ & $121.1(6)$ \\
\hline $\mathrm{N}(5)-\mathrm{C}(32)-\mathrm{C}(33)$ & $120.4(6)$ & $\mathrm{C}(53)-\mathrm{C}(54)-\mathrm{C}(29)$ & $109.6(5)$ \\
\hline$C(36)-C(33)-C(32)$ & $110.4(6)$ & $\mathrm{C}(53)-\mathrm{C}(54)-\mathrm{C}(55)$ & $109.9(6)$ \\
\hline$C(36)-C(33)-C(34)$ & $109.9(6)$ & $C(29)-C(54)-C(55)$ & $109.9(6)$ \\
\hline$C(53)-C(54)-C(56)$ & $109.9(6)$ & $\mathrm{C}(103)-\mathrm{C}(98)-\mathrm{C}(99)$ & $114.7(10)$ \\
\hline$C(29)-C(54)-C(56)$ & $108.6(6)$ & $\mathrm{C}(103)-\mathrm{C}(98)-\mathrm{C}(97)$ & $121.5(13)$ \\
\hline$C(55)-C(54)-C(56)$ & $108.9(6)$ & $\mathrm{C}(99)-\mathrm{C}(98)-\mathrm{C}(97)$ & $123.8(13)$ \\
\hline $\mathrm{O}(1)-\mathrm{C}(57)-\mathrm{C}(58)$ & $105.0(9)$ & $\mathrm{C}(100)-\mathrm{C}(99)-\mathrm{C}(98)$ & $117.9(9)$ \\
\hline $\mathrm{C}(57)-\mathrm{C}(58)-\mathrm{C}(59)$ & $101.8(10)$ & $\mathrm{C}(101)-\mathrm{C}(100)-\mathrm{C}(99)$ & $123.7(12)$ \\
\hline$C(60)-C(59)-C(58)$ & $107.3(10)$ & $C(100)-C(101)-C(102)$ & $118.8(13)$ \\
\hline $\mathrm{C}(59)-\mathrm{C}(60)-\mathrm{O}(1)$ & $107.0(10)$ & $\mathrm{C}(101)-\mathrm{C}(102)-\mathrm{C}(103)$ & $124.3(11)$ \\
\hline $\mathrm{O}(2)-\mathrm{C}(61)-\mathrm{C}(62)$ & $108.4(8)$ & $\mathrm{C}(102)-\mathrm{C}(103)-\mathrm{C}(98)$ & $120.6(10)$ \\
\hline$C(63)-C(62)-C(61)$ & 103.7(9) & $C(105)-C(104)-C(106)$ & $66(2)$ \\
\hline$C(62)-C(63)-C(64)$ & 107.3(9) & $C(105)-C(104)-C(111)$ & $44(2)$ \\
\hline $\mathrm{O}(2)-\mathrm{C}(64)-\mathrm{C}(63)$ & $104.2(8)$ & $\mathrm{C}(106)-\mathrm{C}(104)-\mathrm{C}(111)$ & $108(2)$ \\
\hline $\mathrm{O}(3)-\mathrm{C}(65)-\mathrm{C}(66)$ & $105.8(7)$ & $\mathrm{C}(105)-\mathrm{C}(104)-\mathrm{C}(109)$ & $102(2)$ \\
\hline
\end{tabular}




\begin{tabular}{|c|c|c|c|}
\hline$C(65)-C(66)-C(67)$ & $105.6(7)$ & $C(106)-C(104)-C(109)$ & $37.0(14)$ \\
\hline $\mathrm{C}(68)-\mathrm{C}(67)-\mathrm{C}(66)$ & $104.9(7)$ & $\mathrm{C}(111)-\mathrm{C}(104)-\mathrm{C}(109)$ & $144.6(18)$ \\
\hline $\mathrm{O}(3)-\mathrm{C}(68)-\mathrm{C}(67)$ & $105.6(7)$ & $C(111)-C(105)-C(104)$ & $84(4)$ \\
\hline $\mathrm{C}(70)-\mathrm{C}(69)-\mathrm{O}(4)$ & $106.9(8)$ & $C(111)-C(105)-C(113)$ & $112(5)$ \\
\hline $\mathrm{C}(69)-\mathrm{C}(70)-\mathrm{C}(71)$ & $107.3(8)$ & $C(104)-C(105)-C(113)$ & $137(3)$ \\
\hline $\mathrm{C}(72)-\mathrm{C}(71)-\mathrm{C}(70)$ & $103.0(8)$ & $C(111)-C(105)-C(106)$ & $139(5)$ \\
\hline $\mathrm{C}(71)-\mathrm{C}(72)-\mathrm{O}(4)$ & $105.8(7)$ & $C(104)-C(105)-C(106)$ & $59(3)$ \\
\hline $\mathrm{O}(5)-\mathrm{C}(73)-\mathrm{C}(74)$ & $104.7(9)$ & $C(113)-C(105)-C(106)$ & $88(3)$ \\
\hline $\mathrm{C}(75)-\mathrm{C}(74)-\mathrm{C}(73)$ & $105.3(9)$ & $\mathrm{C}(111)-\mathrm{C}(105)-\mathrm{C}(112)$ & $54(3)$ \\
\hline$C(74)-C(75)-C(76)$ & $102.8(9)$ & $C(104)-C(105)-C(112)$ & $132(4)$ \\
\hline $\mathrm{O}(5)-\mathrm{C}(76)-\mathrm{C}(75)$ & $106.2(8)$ & $C(113)-C(105)-C(112)$ & $60(4)$ \\
\hline $\mathrm{O}(6)-\mathrm{C}(77)-\mathrm{C}(78)$ & $105.0(7)$ & $C(106)-C(105)-C(112)$ & $141(4)$ \\
\hline $\mathrm{C}(77)-\mathrm{C}(78)-\mathrm{C}(79)$ & $103.7(8)$ & $C(111)-C(105)-C(107)$ & $144(5)$ \\
\hline $\mathrm{C}(80)-\mathrm{C}(79)-\mathrm{C}(78)$ & $102.7(7)$ & $C(104)-C(105)-C(107)$ & $108(3)$ \\
\hline $\mathrm{O}(6)-\mathrm{C}(80)-\mathrm{C}(79)$ & $106.1(7)$ & $C(113)-C(105)-C(107)$ & $37(2)$ \\
\hline $\mathrm{O}(7)-\mathrm{C}(81)-\mathrm{C}(82)$ & $104.7(7)$ & $C(106)-C(105)-C(107)$ & $52(2)$ \\
\hline $\mathrm{C}(83)-\mathrm{C}(82)-\mathrm{C}(81)$ & $104.1(7)$ & $C(112)-C(105)-C(107)$ & $97(4)$ \\
\hline $\mathrm{C}(82)-\mathrm{C}(83)-\mathrm{C}(84)$ & $104.3(8)$ & $C(109)-C(106)-C(108)$ & $75(4)$ \\
\hline $\mathrm{O}(7)-\mathrm{C}(84)-\mathrm{C}(83)$ & $105.5(7)$ & $C(109)-C(106)-C(107)$ & $138(4)$ \\
\hline $\mathrm{O}(8)-\mathrm{C}(85)-\mathrm{C}(86)$ & $107.4(6)$ & $C(108)-C(106)-C(107)$ & $64(3)$ \\
\hline $\mathrm{C}(85)-\mathrm{C}(86)-\mathrm{C}(87)$ & $105.2(6)$ & $C(109)-C(106)-C(104)$ & $97(3)$ \\
\hline $\mathrm{C}(88)-\mathrm{C}(87)-\mathrm{C}(86)$ & $103.0(6)$ & $C(108)-C(106)-C(104)$ & $171(3)$ \\
\hline $\mathrm{O}(8)-\mathrm{C}(88)-\mathrm{C}(87)$ & $105.1(6)$ & $C(107)-C(106)-C(104)$ & $123(3)$ \\
\hline $\mathrm{O}(9)-\mathrm{C}(89)-\mathrm{C}(90)$ & $106.4(6)$ & $C(109)-C(106)-C(105)$ & $151(4)$ \\
\hline $\mathrm{C}(89)-\mathrm{C}(90)-\mathrm{C}(91)$ & $102.3(7)$ & $\mathrm{C}(108)-\mathrm{C}(106)-\mathrm{C}(105)$ & $133(4)$ \\
\hline $\mathrm{C}(92)-\mathrm{C}(91)-\mathrm{C}(90)$ & $102.5(6)$ & $C(107)-C(106)-C(105)$ & $71(3)$ \\
\hline $\mathrm{O}(9)-\mathrm{C}(92)-\mathrm{C}(91)$ & $105.8(6)$ & $C(104)-C(106)-C(105)$ & $56(3)$ \\
\hline $\mathrm{O}(10)-\mathrm{C}(93)-\mathrm{C}(94)$ & $107.3(7)$ & $C(110)-C(107)-C(113)$ & $113(6)$ \\
\hline $\mathrm{C}(93)-\mathrm{C}(94)-\mathrm{C}(95)$ & $105.3(7)$ & $C(110)-C(107)-C(106)$ & $126(7)$ \\
\hline $\mathrm{C}(96)-\mathrm{C}(95)-\mathrm{C}(94)$ & $101.7(7)$ & $C(113)-C(107)-C(106)$ & $118(4)$ \\
\hline $\mathrm{O}(10)-\mathrm{C}(96)-\mathrm{C}(95)$ & $104.6(6)$ & $\mathrm{C}(110)-\mathrm{C}(107)-\mathrm{C}(108)$ & $75(5)$ \\
\hline $\mathrm{C}(113)-\mathrm{C}(107)-\mathrm{C}(108)$ & $171(3)$ & $\mathrm{C}(107)-\mathrm{C}(110)-\mathrm{C}(108)$ & $71(5)$ \\
\hline $\mathrm{C}(106)-\mathrm{C}(107)-\mathrm{C}(108)$ & $54(2)$ & $C(107)-C(110)-C(113)$ & $37(5)$ \\
\hline$C(110)-C(107)-C(105)$ & $151(7)$ & $C(108)-C(110)-C(113)$ & $109(5)$ \\
\hline$C(113)-C(107)-C(105)$ & $62(3)$ & $C(105)-C(111)-C(112)$ & $79(4)$ \\
\hline$C(106)-C(107)-C(105)$ & $58(3)$ & $C(105)-C(111)-C(104)$ & $52(3)$ \\
\hline $\mathrm{C}(108)-\mathrm{C}(107)-\mathrm{C}(105)$ & $110(3)$ & $C(112)-C(111)-C(104)$ & $125(3)$ \\
\hline$C(106)-C(108)-C(107)$ & $62(2)$ & $C(111)-C(112)-C(113)$ & $102(3)$ \\
\hline$C(106)-C(108)-C(110)$ & $94(4)$ & $C(111)-C(112)-C(105)$ & $47(2)$ \\
\hline$C(107)-C(108)-C(110)$ & $33(3)$ & $C(113)-C(112)-C(105)$ & $56(2)$ \\
\hline $\mathrm{C}(106)-\mathrm{C}(108)-\mathrm{C}(109)$ & $50(2)$ & $C(107)-C(113)-C(105)$ & $81(4)$ \\
\hline$C(107)-C(108)-C(109)$ & $112(3)$ & $C(107)-C(113)-C(112)$ & $143(5)$ \\
\hline$C(110)-C(108)-C(109)$ & $144(5)$ & $C(105)-C(113)-C(112)$ & $64(3)$ \\
\hline
\end{tabular}




$\begin{array}{lrlr}\mathrm{C}(106)-\mathrm{C}(109)-\mathrm{C}(108) & 55(2) & \mathrm{C}(107)-\mathrm{C}(113)-\mathrm{C}(110) & 29(2) \\ \mathrm{C}(106)-\mathrm{C}(109)-\mathrm{C}(104) & 46(3) & \mathrm{C}(105)-\mathrm{C}(113)-\mathrm{C}(110) & 106(5) \\ \mathrm{C}(108)-\mathrm{C}(109)-\mathrm{C}(104) & 101(2) & \mathrm{C}(112)-\mathrm{C}(113)-\mathrm{C}(110) & 169(5)\end{array}$

Primed labels refer to atoms generated by the following symmetry transformation: $-\mathrm{x}+2$, $-\mathrm{y}+1,-\mathrm{z}+1$ 
Table S12. Anisotropic displacement parameters $\left(\AA^{2} \times 10^{3}\right)$ for $\left[(\mathrm{THF})_{3} \mathrm{Fe}^{\mathrm{II}}(\mu-\mathrm{Cl})_{3}\right.$ $\left.\mathrm{Fe}^{\mathrm{II}}(\mathrm{THF})_{3}\right]\left[(\mathrm{THF})_{4} \mathrm{Fe}^{\mathrm{II}}(\mu-\mathrm{Cl})_{2} \mathrm{Fe}^{\mathrm{II}}(\mathrm{THF})_{4}\right]_{1 / 2}\left[\mathrm{LFe}^{\mathrm{III}}\right]_{2} \cdot 2 \mathrm{PhCH}_{3}$.

\begin{tabular}{|c|c|c|c|c|c|c|}
\hline Atom & $\mathrm{U}_{11}$ & $\mathrm{U}_{22}$ & $\mathrm{U}_{33}$ & $\mathrm{U}_{23}$ & $\mathrm{U}_{13}$ & $\mathrm{U}_{12}$ \\
\hline $\mathrm{Fe}(1)$ & $24(1)$ & $23(1)$ & $24(1)$ & $-1(1)$ & $-2(1)$ & $-8(1)$ \\
\hline $\mathrm{Fe}(2)$ & $20(1)$ & $22(1)$ & $25(1)$ & $2(1)$ & $-4(1)$ & $-4(1)$ \\
\hline $\mathrm{Fe}(3)$ & $29(1)$ & $26(1)$ & $36(1)$ & $-4(1)$ & $-4(1)$ & $-5(1)$ \\
\hline $\mathrm{Fe}(4)$ & $29(1)$ & $28(1)$ & $33(1)$ & $-5(1)$ & $-2(1)$ & $-7(1)$ \\
\hline $\mathrm{Fe}(5)$ & $24(1)$ & $30(1)$ & $27(1)$ & $-2(1)$ & $-6(1)$ & $-4(1)$ \\
\hline $\mathrm{Cl}(1)$ & $50(1)$ & $29(1)$ & $35(1)$ & $-4(1)$ & $-7(1)$ & $-10(1)$ \\
\hline $\mathrm{Cl}(2)$ & $31(1)$ & $35(1)$ & $50(1)$ & $0(1)$ & $-10(1)$ & $-8(1)$ \\
\hline $\mathrm{Cl}(3)$ & $30(1)$ & $41(1)$ & $44(1)$ & $-6(1)$ & $4(1)$ & $-1(1)$ \\
\hline $\mathrm{Cl}(4)$ & $24(1)$ & $46(1)$ & $33(1)$ & $-11(1)$ & $-3(1)$ & $-3(1)$ \\
\hline $\mathrm{O}(1)$ & $40(3)$ & $57(4)$ & $31(3)$ & $-13(3)$ & $-4(2)$ & $-6(3)$ \\
\hline $\mathrm{O}(2)$ & $35(3)$ & $45(3)$ & $43(3)$ & $-12(2)$ & $1(2)$ & $-20(2)$ \\
\hline $\mathrm{O}(3)$ & $41(3)$ & $32(3)$ & $40(3)$ & $1(2)$ & $3(2)$ & $-6(2)$ \\
\hline $\mathrm{O}(4)$ & $41(3)$ & $26(3)$ & $56(4)$ & $-11(2)$ & $-14(3)$ & $1(2)$ \\
\hline $\mathrm{O}(5)$ & $42(3)$ & $36(3)$ & $50(3)$ & $-1(2)$ & $-15(3)$ & $-12(2)$ \\
\hline $\mathrm{O}(6)$ & $32(3)$ & $31(3)$ & $51(3)$ & $4(2)$ & $8(2)$ & $-6(2)$ \\
\hline $\mathrm{O}(7)$ & $42(3)$ & $35(3)$ & $40(3)$ & $1(2)$ & $-12(2)$ & $-15(2)$ \\
\hline $\mathrm{O}(8)$ & $40(3)$ & $30(3)$ & $25(3)$ & $-1(2)$ & $-1(2)$ & $-3(2)$ \\
\hline $\mathrm{O}(9)$ & $32(3)$ & $40(3)$ & $20(2)$ & $-5(2)$ & $-1(2)$ & $-12(2)$ \\
\hline $\mathrm{O}(10)$ & $17(2)$ & $51(3)$ & $33(3)$ & $-6(2)$ & $-3(2)$ & $1(2)$ \\
\hline $\mathrm{N}(1)$ & $27(3)$ & $24(3)$ & $22(3)$ & $-2(2)$ & $-5(2)$ & $-4(2)$ \\
\hline $\mathrm{N}(2)$ & $28(3)$ & $27(3)$ & $33(3)$ & $0(3)$ & $-4(3)$ & $-11(3)$ \\
\hline $\mathrm{N}(3)$ & $27(3)$ & $27(3)$ & $25(3)$ & $1(2)$ & $-2(2)$ & $-4(3)$ \\
\hline $\mathrm{N}(4)$ & $24(3)$ & $30(3)$ & $24(3)$ & $1(2)$ & $-4(2)$ & $-9(3)$ \\
\hline $\mathrm{N}(5)$ & $22(3)$ & $27(3)$ & $23(3)$ & $-1(2)$ & $-2(2)$ & $-2(3)$ \\
\hline $\mathrm{N}(6)$ & $23(3)$ & $21(3)$ & $30(3)$ & $-2(2)$ & $-3(2)$ & $-3(2)$ \\
\hline $\mathrm{N}(7)$ & $20(3)$ & $28(3)$ & $28(3)$ & $2(2)$ & $0(2)$ & $-5(2)$ \\
\hline $\mathrm{N}(8)$ & $22(3)$ & $29(3)$ & $24(3)$ & $2(2)$ & $-2(2)$ & $-7(3)$ \\
\hline $\mathrm{C}(1)$ & $31(4)$ & $21(4)$ & $36(4)$ & $2(3)$ & $-17(3)$ & $-4(3)$ \\
\hline $\mathrm{C}(2)$ & $35(4)$ & $22(4)$ & $40(4)$ & $-7(3)$ & $-4(3)$ & $3(3)$ \\
\hline $\mathrm{C}(3)$ & $35(4)$ & $43(5)$ & $25(4)$ & $-15(3)$ & $-1(3)$ & $-5(4)$ \\
\hline$C(4)$ & $24(4)$ & $38(4)$ & $22(4)$ & $0(3)$ & $-2(3)$ & $-9(3)$ \\
\hline$C(5)$ & $30(4)$ & $37(4)$ & $21(4)$ & $3(3)$ & $-1(3)$ & $-12(3)$ \\
\hline$C(6)$ & $33(4)$ & $38(4)$ & $33(4)$ & $-1(3)$ & $-9(3)$ & $-3(3)$ \\
\hline $\mathrm{C}(7)$ & $48(5)$ & $50(5)$ & $27(4)$ & $6(4)$ & $-6(4)$ & $-17(4)$ \\
\hline $\mathrm{C}(8)$ & $24(4)$ & $34(4)$ & $28(4)$ & $9(3)$ & $-7(3)$ & $-6(3)$ \\
\hline $\mathrm{C}(9)$ & $46(5)$ & $42(4)$ & $26(4)$ & $12(3)$ & $-6(3)$ & $-23(4)$ \\
\hline $\mathrm{C}(10)$ & $42(5)$ & $30(4)$ & $51(5)$ & $7(4)$ & $-16(4)$ & $-24(4)$ \\
\hline
\end{tabular}




\begin{tabular}{|c|c|c|c|c|c|c|}
\hline$C(11)$ & $30(4)$ & $24(4)$ & $30(4)$ & $-1(3)$ & $-5(3)$ & $-10(3)$ \\
\hline$C(12)$ & $50(5)$ & $24(4)$ & $26(4)$ & $-1(3)$ & $-13(3)$ & $-12(3)$ \\
\hline$C(13)$ & $53(5)$ & $32(4)$ & $45(5)$ & $-1(4)$ & $-9(4)$ & $-16(4)$ \\
\hline$C(14)$ & $43(5)$ & $38(4)$ & $43(5)$ & $2(4)$ & $-13(4)$ & $-15(4)$ \\
\hline$C(15)$ & $32(4)$ & $21(4)$ & $26(4)$ & $0(3)$ & $-4(3)$ & $0(3)$ \\
\hline$C(16)$ & $54(5)$ & $26(4)$ & $31(4)$ & $0(3)$ & $-9(4)$ & $-2(4)$ \\
\hline$C(17)$ & $38(4)$ & $29(4)$ & $38(5)$ & $4(3)$ & $6(4)$ & $-2(4)$ \\
\hline$C(18)$ & $28(4)$ & $34(4)$ & $29(4)$ & $4(3)$ & $-4(3)$ & $-3(3)$ \\
\hline$C(19)$ & $22(4)$ & $45(4)$ & $32(4)$ & $4(3)$ & $1(3)$ & $-13(3)$ \\
\hline$C(20)$ & $34(4)$ & $58(5)$ & $46(5)$ & $3(4)$ & $-12(4)$ & $-17(4)$ \\
\hline$C(21)$ & $33(4)$ & $53(5)$ & $46(5)$ & $7(4)$ & $-1(4)$ & $-17(4)$ \\
\hline$C(22)$ & $25(4)$ & $39(4)$ & $34(4)$ & $-1(3)$ & $-8(3)$ & $-17(3)$ \\
\hline $\mathrm{C}(23)$ & $41(5)$ & $49(5)$ & $40(5)$ & $4(4)$ & $1(4)$ & $-25(4)$ \\
\hline$C(24)$ & $54(5)$ & $33(4)$ & $44(5)$ & $3(4)$ & $-7(4)$ & $-22(4)$ \\
\hline$C(25)$ & $42(4)$ & $25(4)$ & $28(4)$ & $-1(3)$ & $-5(3)$ & $-17(3)$ \\
\hline$C(26)$ & $43(5)$ & $25(4)$ & $30(4)$ & $6(3)$ & $-11(3)$ & $-9(3)$ \\
\hline$C(27)$ & $63(6)$ & $21(4)$ & $51(5)$ & $4(4)$ & $-7(4)$ & $-8(4)$ \\
\hline$C(28)$ & $37(4)$ & $38(4)$ & $36(4)$ & $1(3)$ & $-10(3)$ & $-9(4)$ \\
\hline$C(29)$ & $22(4)$ & $36(4)$ & $26(4)$ & $-9(3)$ & $-5(3)$ & $-6(3)$ \\
\hline$C(30)$ & $23(4)$ & $49(5)$ & $42(5)$ & $-12(4)$ & $-5(3)$ & $-11(4)$ \\
\hline $\mathrm{C}(31)$ & $18(4)$ & $44(5)$ & $50(5)$ & $-12(4)$ & $-1(3)$ & $-1(3)$ \\
\hline$C(32)$ & $26(4)$ & $30(4)$ & $25(4)$ & $-6(3)$ & $-9(3)$ & $0(3)$ \\
\hline$C(33)$ & $28(4)$ & $26(4)$ & $33(4)$ & $-1(3)$ & $0(3)$ & $-1(3)$ \\
\hline$C(34)$ & $42(5)$ & $23(4)$ & $49(5)$ & $-7(3)$ & $-8(4)$ & $3(3)$ \\
\hline$C(35)$ & $43(5)$ & $36(4)$ & $35(4)$ & $-7(3)$ & $-7(4)$ & $-7(4)$ \\
\hline$C(36)$ & $29(4)$ & $25(4)$ & $42(4)$ & $3(3)$ & $-16(3)$ & $-5(3)$ \\
\hline$C(37)$ & $39(4)$ & $23(4)$ & $44(5)$ & $10(3)$ & $-22(4)$ & $-7(3)$ \\
\hline$C(38)$ & $41(4)$ & $31(4)$ & $33(4)$ & $12(3)$ & $-12(3)$ & $-16(4)$ \\
\hline C(39) & $36(4)$ & $33(4)$ & $24(4)$ & $-1(3)$ & $-5(3)$ & $-17(3)$ \\
\hline$C(40)$ & $32(4)$ & $30(4)$ & $26(4)$ & $-1(3)$ & $0(3)$ & $-14(3)$ \\
\hline$C(41)$ & $34(4)$ & $34(4)$ & $30(4)$ & $-9(3)$ & $-2(3)$ & $-11(3)$ \\
\hline$C(42)$ & $35(4)$ & $49(5)$ & $38(4)$ & $4(4)$ & $-4(3)$ & $-21(4)$ \\
\hline$C(43)$ & $35(4)$ & $30(4)$ & $29(4)$ & $-9(3)$ & $0(3)$ & $-10(3)$ \\
\hline$C(44)$ & $25(4)$ & $42(4)$ & $39(5)$ & $-2(4)$ & $6(3)$ & $-7(3)$ \\
\hline$C(45)$ & $25(4)$ & $41(4)$ & $42(5)$ & $0(4)$ & $-10(3)$ & $-5(3)$ \\
\hline$C(46)$ & $22(4)$ & $21(4)$ & $38(4)$ & $1(3)$ & $-12(3)$ & $3(3)$ \\
\hline$C(47)$ & $31(4)$ & $28(4)$ & $28(4)$ & $1(3)$ & $-10(3)$ & $-1(3)$ \\
\hline$C(48)$ & $40(5)$ & $41(5)$ & $42(5)$ & $2(4)$ & $-21(4)$ & $-5(4)$ \\
\hline C(49) & $37(4)$ & $39(4)$ & $43(5)$ & $-2(4)$ & $-15(4)$ & $-8(4)$ \\
\hline$C(50)$ & $25(4)$ & $27(4)$ & $21(4)$ & $-3(3)$ & $-2(3)$ & $-1(3)$ \\
\hline$C(51)$ & $37(4)$ & $28(4)$ & $28(4)$ & $6(3)$ & $-6(3)$ & $-3(3)$ \\
\hline$C(52)$ & $43(5)$ & $28(4)$ & $32(4)$ & $3(3)$ & $-1(3)$ & $-15(3)$ \\
\hline$C(53)$ & $36(4)$ & $29(4)$ & $24(4)$ & $-7(3)$ & $-4(3)$ & $-12(3)$ \\
\hline
\end{tabular}




\begin{tabular}{|c|c|c|c|c|c|c|}
\hline$C(54)$ & $33(4)$ & $26(4)$ & $26(4)$ & $2(3)$ & $-9(3)$ & $-13(3)$ \\
\hline$C(55)$ & $38(4)$ & $39(4)$ & $30(4)$ & $-7(3)$ & $-8(3)$ & $-10(4)$ \\
\hline$C(56)$ & $39(5)$ & $47(5)$ & $42(5)$ & $-2(4)$ & $-5(4)$ & $-21(4)$ \\
\hline$C(57)$ & $47(6)$ & $93(8)$ & $53(6)$ & $4(5)$ & $-20(5)$ & $-20(5)$ \\
\hline $\mathrm{C}(58)$ & $125(12)$ & $113(11)$ & $106(11)$ & $-14(8)$ & $-50(9)$ & $-59(10)$ \\
\hline$C(59)$ & $114(13)$ & 181(17) & $180(17)$ & $-141(14)$ & $-57(12)$ & $8(11)$ \\
\hline$C(60)$ & $63(7)$ & $141(11)$ & $44(6)$ & $-43(7)$ & $-4(5)$ & $7(7)$ \\
\hline$C(61)$ & 107(9) & $111(9)$ & $72(7)$ & $-41(7)$ & $21(6)$ & $-85(8)$ \\
\hline$C(62)$ & $80(8)$ & $108(9)$ & $60(7)$ & $-13(6)$ & $-11(6)$ & $-58(7)$ \\
\hline$C(63)$ & $49(7)$ & $151(13)$ & $129(11)$ & $-63(10)$ & $16(7)$ & $-52(8)$ \\
\hline$C(64)$ & $58(6)$ & $84(7)$ & $59(6)$ & $-27(5)$ & $24(5)$ & $-43(6)$ \\
\hline$C(65)$ & $32(4)$ & $49(5)$ & $42(5)$ & $-3(4)$ & $8(4)$ & $-3(4)$ \\
\hline$C(66)$ & $82(7)$ & $49(6)$ & $63(6)$ & $-15(5)$ & $14(5)$ & $-11(5)$ \\
\hline$C(67)$ & $73(7)$ & $28(5)$ & $94(8)$ & $8(5)$ & $6(6)$ & $-3(5)$ \\
\hline$C(68)$ & $61(6)$ & $44(5)$ & $46(5)$ & $11(4)$ & $5(4)$ & $0(4)$ \\
\hline$C(69)$ & $76(7)$ & $38(5)$ & $88(8)$ & $-23(5)$ & $-47(6)$ & $21(5)$ \\
\hline$C(70)$ & $70(7)$ & $53(6)$ & $82(8)$ & $-27(5)$ & $-15(6)$ & $11(5)$ \\
\hline $\mathrm{C}(71)$ & $87(8)$ & $46(6)$ & $70(7)$ & $-16(5)$ & $-24(6)$ & $-8(5)$ \\
\hline$C(72)$ & $76(7)$ & $47(6)$ & $88(8)$ & $-17(5)$ & $-42(6)$ & $-8(5)$ \\
\hline$C(73)$ & $77(7)$ & $65(6)$ & $44(6)$ & $-1(5)$ & $-21(5)$ & $-21(5)$ \\
\hline$C(74)$ & $80(9)$ & $102(10)$ & $114(11)$ & $-37(8)$ & $-52(8)$ & $-12(7)$ \\
\hline$C(75)$ & $87(8)$ & $42(6)$ & 101(9) & $2(5)$ & $-39(7)$ & $-29(6)$ \\
\hline$C(76)$ & $53(6)$ & $44(5)$ & $77(7)$ & $13(5)$ & $-31(5)$ & $-15(4)$ \\
\hline$C(77)$ & $38(5)$ & $55(5)$ & $40(5)$ & $-10(4)$ & $3(4)$ & $-8(4)$ \\
\hline$C(78)$ & $62(6)$ & $84(7)$ & $48(6)$ & $-28(5)$ & $16(5)$ & $-41(6)$ \\
\hline$C(79)$ & $106(8)$ & $62(6)$ & $38(5)$ & $4(4)$ & $-2(5)$ & $-56(6)$ \\
\hline$C(80)$ & $72(7)$ & $37(5)$ & $85(7)$ & $16(5)$ & $7(6)$ & $-20(5)$ \\
\hline$C(81)$ & $39(5)$ & $57(5)$ & $57(6)$ & $-12(4)$ & $-6(4)$ & $-26(4)$ \\
\hline$C(82)$ & $79(7)$ & $79(7)$ & $65(7)$ & $-9(5)$ & $7(6)$ & $-54(6)$ \\
\hline$C(83)$ & $105(9)$ & $53(6)$ & $46(6)$ & $7(4)$ & $-11(5)$ & $-35(6)$ \\
\hline $\mathrm{C}(84)$ & $63(6)$ & $38(5)$ & $36(5)$ & $8(4)$ & $-16(4)$ & $-14(4)$ \\
\hline$C(85)$ & $54(5)$ & $36(5)$ & $34(4)$ & $-12(4)$ & $-1(4)$ & $-3(4)$ \\
\hline$C(86)$ & $41(5)$ & $30(4)$ & $49(5)$ & $-2(4)$ & $2(4)$ & $-5(4)$ \\
\hline$C(87)$ & $42(5)$ & $46(5)$ & $40(5)$ & $7(4)$ & $-13(4)$ & $-15(4)$ \\
\hline$C(88)$ & $53(5)$ & $42(5)$ & $31(4)$ & $-8(4)$ & $-5(4)$ & $-15(4)$ \\
\hline C(89) & $46(5)$ & $58(5)$ & $37(5)$ & $-7(4)$ & $-9(4)$ & $-19(4)$ \\
\hline$C(90)$ & $73(6)$ & $43(5)$ & $48(5)$ & $1(4)$ & $-21(5)$ & $-25(5)$ \\
\hline $\mathrm{C}(91)$ & $64(6)$ & $48(5)$ & $27(4)$ & $-5(4)$ & $-5(4)$ & $-14(4)$ \\
\hline$C(92)$ & $46(5)$ & $32(4)$ & $31(4)$ & $-1(3)$ & $0(3)$ & $-8(4)$ \\
\hline C(93) & $31(5)$ & $89(7)$ & $46(5)$ & $0(5)$ & $6(4)$ & $4(5)$ \\
\hline$C(94)$ & $27(5)$ & $87(7)$ & $52(6)$ & $-10(5)$ & $-3(4)$ & $4(5)$ \\
\hline$C(95)$ & $32(4)$ & $50(5)$ & $46(5)$ & $-10(4)$ & $-7(4)$ & $-2(4)$ \\
\hline$C(96)$ & $29(4)$ & $55(5)$ & $35(4)$ & $-13(4)$ & $-11(3)$ & $-2(4)$ \\
\hline
\end{tabular}




\begin{tabular}{rrrrrrr}
$\mathrm{C}(97)$ & $124(12)$ & $320(20)$ & $66(8)$ & $74(11)$ & $-44(8)$ & $-161(15)$ \\
$\mathrm{C}(98)$ & $81(7)$ & $121(10)$ & $29(5)$ & $15(6)$ & $-1(5)$ & $-71(7)$ \\
$\mathrm{C}(99)$ & $158(13)$ & $80(8)$ & $51(7)$ & $-37(6)$ & $40(8)$ & $-89(9)$ \\
$\mathrm{C}(100)$ & $97(10)$ & $65(8)$ & $65(8)$ & $-3(6)$ & $10(7)$ & $-8(7)$ \\
$\mathrm{C}(101)$ & $76(8)$ & $89(9)$ & $56(7)$ & $2(6)$ & $5(6)$ & $-18(7)$ \\
$\mathrm{C}(102)$ & $73(7)$ & $52(6)$ & $67(7)$ & $5(5)$ & $-13(6)$ & $-12(6)$ \\
$\mathrm{C}(103)$ & $56(6)$ & $40(5)$ & $53(6)$ & $4(4)$ & $-9(5)$ & $-6(4)$ \\
$\mathrm{C}(104)$ & $70(9)$ & $100(13)$ & $200(20)$ & $-20(14)$ & $-18(11)$ & $-27(9)$ \\
$\mathrm{C}(105)$ & $250(60)$ & $130(30)$ & $100(30)$ & $70(30)$ & $-100(30)$ & $-80(40)$ \\
$\mathrm{C}(106)$ & $59(15)$ & $90(20)$ & $140(30)$ & $-50(20)$ & $-51(17)$ & $11(15)$ \\
$\mathrm{C}(107)$ & $111(18)$ & $7(9)$ & $52(15)$ & $13(9)$ & $-38(13)$ & $-9(10)$ \\
$\mathrm{C}(108)$ & $490(60)$ & $220(40)$ & $180(30)$ & $80(30)$ & $-220(40)$ & $-180(40)$ \\
$\mathrm{C}(109)$ & $109(15)$ & $136(18)$ & $290(30)$ & $-70(20)$ & $-20(17)$ & $-41(13)$ \\
$\mathrm{C}(110)$ & $240(60)$ & $150(50)$ & $160(50)$ & $-50(50)$ & $-60(40)$ & $-40(50)$ \\
$\mathrm{C}(111)$ & $64(16)$ & $76(16)$ & $120(20)$ & $-11(18)$ & $-59(16)$ & $-28(14)$ \\
$\mathrm{C}(112)$ & $55(16)$ & $260(50)$ & $110(30)$ & $-110(30)$ & $-8(17)$ & $-40(20)$ \\
$\mathrm{C}(113)$ & $260(40)$ & $350(50)$ & $210(30)$ & $30(40)$ & $-130(30)$ & $-220(40)$ \\
\hline
\end{tabular}

The anisotropic displacement factor exponent takes the form: $-2 \pi^{2}\left[\mathrm{~h}^{2} \mathrm{a}^{* 2} \mathrm{U}_{11}+\ldots+\right.$ $\left.2 \mathrm{hka} * \mathrm{~b} * \mathrm{U}_{12}\right]$ 
Table S13. Hydrogen coordinates $\left(\times 10^{4}\right)$ and isotropic displacement parameters $\left(\AA^{2} \times 10^{3}\right)$ for $\left[(\mathrm{THF})_{3} \mathrm{Fe}^{\mathrm{II}}(\mu-\mathrm{Cl})_{3}\right.$ $\left.\mathrm{Fe}^{\mathrm{II}}(\mathrm{THF})_{3}\right]\left[(\mathrm{THF})_{4} \mathrm{Fe}^{\mathrm{II}}(\mu-\mathrm{Cl})_{2} \mathrm{Fe}^{\mathrm{II}}(\mathrm{THF})_{4}\right]_{1 / 2}\left[\mathrm{LFe}^{\mathrm{IIII}}\right]_{2} \bullet 2 \mathrm{PhCH}_{3}$.

\begin{tabular}{|c|c|c|c|c|}
\hline Atom & $\mathrm{x}$ & $\mathrm{y}$ & z & $\mathrm{U}_{\mathrm{eq}}$ \\
\hline $\mathrm{H}(2)$ & 6497 & 8615 & 9251 & 42 \\
\hline $\mathrm{H}(3)$ & 6499 & 7336 & 9862 & 43 \\
\hline $\mathrm{H}(6 \mathrm{~A})$ & 9197 & 4571 & 9819 & 55 \\
\hline $\mathrm{H}(6 \mathrm{~B})$ & 8999 & 5642 & 9884 & 55 \\
\hline $\mathrm{H}(6 \mathrm{C})$ & 9502 & 5199 & 9385 & 55 \\
\hline $\mathrm{H}(7 \mathrm{~A})$ & 6292 & 5519 & 9946 & 63 \\
\hline $\mathrm{H}(7 \mathrm{~B})$ & 7046 & 5858 & 10219 & 63 \\
\hline $\mathrm{H}(7 \mathrm{C})$ & 7321 & 4775 & 10154 & 63 \\
\hline $\mathrm{H}(9)$ & 7124 & 3810 & 9568 & 44 \\
\hline $\mathrm{H}(10)$ & 7524 & 3051 & 8795 & 45 \\
\hline $\mathrm{H}(13 \mathrm{~A})$ & 9039 & 2585 & 8016 & 64 \\
\hline $\mathrm{H}(13 \mathrm{~B})$ & 8666 & 3026 & 7527 & 64 \\
\hline $\mathrm{H}(13 \mathrm{C})$ & 7753 & 2992 & 7966 & 64 \\
\hline $\mathrm{H}(14 \mathrm{~A})$ & 6822 & 4696 & 7868 & 60 \\
\hline $\mathrm{H}(14 \mathrm{~B})$ & 7736 & 4688 & 7424 & 60 \\
\hline $\mathrm{H}(14 \mathrm{C})$ & 7519 & 5384 & 7833 & 60 \\
\hline $\mathrm{H}(16)$ & 10453 & 3176 & 7354 & 48 \\
\hline $\mathrm{H}(17)$ & 11920 & 3949 & 7300 & 47 \\
\hline $\mathrm{H}(20 \mathrm{~A})$ & 11080 & 5144 & 8674 & 68 \\
\hline $\mathrm{H}(20 \mathrm{~B})$ & 12102 & 5517 & 8532 & 68 \\
\hline $\mathrm{H}(20 \mathrm{C})$ & 12246 & 4474 & 8430 & 68 \\
\hline $\mathrm{H}(21 \mathrm{~A})$ & 12955 & 4750 & 7615 & 67 \\
\hline $\mathrm{H}(21 \mathrm{~B})$ & 12777 & 5803 & 7713 & 67 \\
\hline $\mathrm{H}(21 \mathrm{C})$ & 12245 & 5576 & 7303 & 67 \\
\hline $\mathrm{H}(23)$ & 11426 & 7304 & 7685 & 50 \\
\hline $\mathrm{H}(24)$ & 9601 & 8597 & 7888 & 50 \\
\hline $\mathrm{H}(27 \mathrm{~A})$ & 6664 & 9307 & 8513 & 71 \\
\hline $\mathrm{H}(27 \mathrm{~B})$ & 7504 & 9322 & 8045 & 71 \\
\hline $\mathrm{H}(27 \mathrm{C})$ & 7926 & 9181 & 8540 & 71 \\
\hline $\mathrm{H}(28 \mathrm{~A})$ & 7175 & 7138 & 7992 & 56 \\
\hline $\mathrm{H}(28 \mathrm{~B})$ & 7228 & 8044 & 7685 & 56 \\
\hline $\mathrm{H}(28 \mathrm{C})$ & 6246 & 8122 & 8110 & 56 \\
\hline $\mathrm{H}(30)$ & 2187 & 1623 & 6374 & 45 \\
\hline $\mathrm{H}(31)$ & 2163 & 3276 & 6369 & 47 \\
\hline $\mathrm{H}(34 \mathrm{~A})$ & 3736 & 4951 & 6067 & 62 \\
\hline $\mathrm{H}(34 \mathrm{~B})$ & 2884 & 4538 & 6395 & 62 \\
\hline
\end{tabular}




\begin{tabular}{|c|c|c|c|}
\hline $\mathrm{H}(34 \mathrm{C})$ & 3045 & 4463 & 5842 \\
\hline $\mathrm{H}(35 \mathrm{~A})$ & 5448 & 2881 & 6607 \\
\hline $\mathrm{H}(35 \mathrm{~B})$ & 4285 & 3547 & 6856 \\
\hline $\mathrm{H}(35 \mathrm{C})$ & 5163 & 3981 & 6566 \\
\hline $\mathrm{H}(37)$ & 5033 & 4695 & 5393 \\
\hline $\mathrm{H}(38)$ & 6760 & 3869 & 4830 \\
\hline $\mathrm{H}(41 \mathrm{~A})$ & 7840 & 720 & 4628 \\
\hline $\mathrm{H}(41 \mathrm{~B})$ & 6862 & 1632 & 4500 \\
\hline $\mathrm{H}(41 \mathrm{C})$ & 6692 & 1050 & 4973 \\
\hline $\mathrm{H}(42 \mathrm{~A})$ & 8783 & 2573 & 4809 \\
\hline $\mathrm{H}(42 \mathrm{~B})$ & 8070 & 2591 & 4411 \\
\hline $\mathrm{H}(42 \mathrm{C})$ & 9076 & 1676 & 4509 \\
\hline $\mathrm{H}(44)$ & 9954 & 924 & 5180 \\
\hline $\mathrm{H}(45)$ & 10141 & -43 & 5939 \\
\hline $\mathrm{H}(48 \mathrm{~A})$ & 9225 & -1272 & 6464 \\
\hline $\mathrm{H}(48 \mathrm{~B})$ & 8749 & -996 & 6993 \\
\hline $\mathrm{H}(48 \mathrm{C})$ & 9622 & -563 & 6697 \\
\hline $\mathrm{H}(49 \mathrm{~A})$ & 8247 & 1052 & 6892 \\
\hline $\mathrm{H}(49 \mathrm{~B})$ & 7464 & 534 & 7192 \\
\hline $\mathrm{H}(49 \mathrm{C})$ & 6999 & 1299 & 6799 \\
\hline $\mathrm{H}(51)$ & 7358 & -1576 & 6813 \\
\hline $\mathrm{H}(52)$ & 5476 & -1462 & 6627 \\
\hline $\mathrm{H}(55 \mathrm{~A})$ & 5174 & 388 & 5435 \\
\hline $\mathrm{H}(55 \mathrm{~B})$ & 3960 & 348 & 5439 \\
\hline $\mathrm{H}(55 \mathrm{C})$ & 4958 & -582 & 5525 \\
\hline $\mathrm{H}(56 \mathrm{~A})$ & 3836 & -889 & 6272 \\
\hline $\mathrm{H}(56 \mathrm{~B})$ & 2854 & 43 & 6172 \\
\hline $\mathrm{H}(56 \mathrm{C})$ & 3334 & -109 & 6653 \\
\hline $\mathrm{H}(57 \mathrm{~A})$ & 4439 & 8440 & 9747 \\
\hline $\mathrm{H}(57 \mathrm{~B})$ & 4101 & 7957 & 10235 \\
\hline $\mathrm{H}(58 \mathrm{~A})$ & 3798 & 9866 & 10045 \\
\hline $\mathrm{H}(58 \mathrm{~B})$ & 4255 & 9240 & 10490 \\
\hline $\mathrm{H}(59 \mathrm{~A})$ & 2562 & 9336 & 10844 \\
\hline $\mathrm{H}(59 \mathrm{~B})$ & 2180 & 10259 & 10520 \\
\hline $\mathrm{H}(60 \mathrm{~A})$ & 1377 & 9628 & 10143 \\
\hline $\mathrm{H}(60 \mathrm{~B})$ & 1703 & 8725 & 10485 \\
\hline $\mathrm{H}(61 \mathrm{~A})$ & 1976 & 6866 & 8923 \\
\hline $\mathrm{H}(61 \mathrm{~B})$ & 1128 & 7783 & 8714 \\
\hline $\mathrm{H}(62 \mathrm{~A})$ & -66 & 7085 & 8964 \\
\hline $\mathrm{H}(62 \mathrm{~B})$ & 655 & 6465 & 9349 \\
\hline $\mathrm{H}(63 \mathrm{~A})$ & -608 & 7472 & 9807 \\
\hline $\mathrm{H}(63 \mathrm{~B})$ & -805 & 8277 & 9406 \\
\hline $\mathrm{H}(64 \mathrm{~A})$ & 225 & 8789 & 9772 \\
\hline
\end{tabular}




\begin{tabular}{|c|c|c|c|}
\hline H(64B) & 806 & 7782 & 10002 \\
\hline $\mathrm{H}(65 \mathrm{~A})$ & 5423 & 7337 & 8843 \\
\hline $\mathrm{H}(65 \mathrm{~B})$ & 4608 & 7563 & 8458 \\
\hline $\mathrm{H}(66 \mathrm{~A})$ & 5905 & 5887 & 8649 \\
\hline $\mathrm{H}(66 \mathrm{~B})$ & 4826 & 6091 & 8402 \\
\hline $\mathrm{H}(67 \mathrm{~A})$ & 3965 & 5597 & 9036 \\
\hline $\mathrm{H}(67 \mathrm{~B})$ & 5078 & 5321 & 9268 \\
\hline $\mathrm{H}(68 \mathrm{~A})$ & 3296 & 6621 & 9594 \\
\hline $\mathrm{H}(68 \mathrm{~B})$ & 4503 & 6634 & 9644 \\
\hline $\mathrm{H}(69 \mathrm{~A})$ & 871 & 12796 & 8379 \\
\hline H(69B) & 249 & 12193 & 8722 \\
\hline $\mathrm{H}(70 \mathrm{~A})$ & 76 & 13002 & 9308 \\
\hline $\mathrm{H}(70 \mathrm{~B})$ & 356 & 13751 & 8931 \\
\hline $\mathrm{H}(71 \mathrm{~A})$ & 2127 & 13314 & 9057 \\
\hline $\mathrm{H}(71 \mathrm{~B})$ & 1593 & 13035 & 9565 \\
\hline $\mathrm{H}(72 \mathrm{~A})$ & 2138 & 11549 & 9409 \\
\hline $\mathrm{H}(72 \mathrm{~B})$ & 3110 & 11816 & 9073 \\
\hline $\mathrm{H}(73 \mathrm{~A})$ & 1972 & 10357 & 7494 \\
\hline $\mathrm{H}(73 \mathrm{~B})$ & 1090 & 11374 & 7421 \\
\hline $\mathrm{H}(74 \mathrm{~A})$ & 225 & 10559 & 7123 \\
\hline $\mathrm{H}(74 \mathrm{~B})$ & 754 & 9640 & 7432 \\
\hline $\mathrm{H}(75 \mathrm{~A})$ & -970 & 10145 & 7815 \\
\hline $\mathrm{H}(75 \mathrm{~B})$ & -1077 & 11219 & 7713 \\
\hline $\mathrm{H}(76 \mathrm{~A})$ & -639 & 11040 & 8440 \\
\hline $\mathrm{H}(76 \mathrm{~B})$ & 84 & 9963 & 8377 \\
\hline $\mathrm{H}(77 \mathrm{~A})$ & 4142 & 9683 & 7491 \\
\hline H(77B) & 4628 & 9540 & 7975 \\
\hline $\mathrm{H}(78 \mathrm{~A})$ & 5552 & 10223 & 7265 \\
\hline $\mathrm{H}(78 \mathrm{~B})$ & 5466 & 10602 & 7772 \\
\hline $\mathrm{H}(79 \mathrm{~A})$ & 4592 & 11946 & 7382 \\
\hline H(79B) & 4032 & 11432 & 7092 \\
\hline $\mathrm{H}(80 \mathrm{~A})$ & 3394 & 11952 & 8030 \\
\hline $\mathrm{H}(80 \mathrm{~B})$ & 2604 & 11975 & 7658 \\
\hline $\mathrm{H}(81 \mathrm{~A})$ & 8376 & 3582 & 5486 \\
\hline $\mathrm{H}(81 \mathrm{~B})$ & 7322 & 4323 & 5775 \\
\hline $\mathrm{H}(82 \mathrm{~A})$ & 7531 & 3469 & 6447 \\
\hline $\mathrm{H}(82 \mathrm{~B})$ & 8000 & 2642 & 6085 \\
\hline $\mathrm{H}(83 \mathrm{~A})$ & 9791 & 2478 & 6114 \\
\hline $\mathrm{H}(83 \mathrm{~B})$ & 9248 & 2745 & 6643 \\
\hline $\mathrm{H}(84 \mathrm{~A})$ & 9280 & 4195 & 6528 \\
\hline $\mathrm{H}(84 \mathrm{~B})$ & 10277 & 3739 & 6120 \\
\hline $\mathrm{H}(85 \mathrm{~A})$ & 8788 & 7815 & 5830 \\
\hline $\mathrm{H}(85 \mathrm{~B})$ & 7612 & 7714 & 5802 \\
\hline
\end{tabular}




\begin{tabular}{|c|c|c|c|c|}
\hline $\mathrm{H}(86 \mathrm{~A})$ & 8499 & 9000 & 5334 & 51 \\
\hline $\mathrm{H}(86 \mathrm{~B})$ & 7229 & 9040 & 5410 & 51 \\
\hline $\mathrm{H}(87 \mathrm{~A})$ & 7468 & 8264 & 4754 & 51 \\
\hline H(87B) & 8408 & 8738 & 4593 & 51 \\
\hline $\mathrm{H}(88 \mathrm{~A})$ & 8980 & 6977 & 4609 & 50 \\
\hline $\mathrm{H}(88 \mathrm{~B})$ & 9785 & 7442 & 4775 & 50 \\
\hline $\mathrm{H}(89 \mathrm{~A})$ & 10497 & 6405 & 6022 & 55 \\
\hline H(89B) & 10782 & 5391 & 6272 & 55 \\
\hline $\mathrm{H}(90 \mathrm{~A})$ & 9762 & 7126 & 6699 & 62 \\
\hline $\mathrm{H}(90 \mathrm{~B})$ & 10652 & 6207 & 6893 & 62 \\
\hline $\mathrm{H}(91 \mathrm{~A})$ & 9347 & 5488 & 7116 & 57 \\
\hline H(91B) & 8619 & 6547 & 7237 & 57 \\
\hline $\mathrm{H}(92 \mathrm{~A})$ & 8011 & 5712 & 6670 & 46 \\
\hline H(92B) & 7842 & 6797 & 6580 & 46 \\
\hline H(93A) & 6481 & 6691 & 6179 & 76 \\
\hline H(93B) & 6231 & 5767 & 6100 & 76 \\
\hline $\mathrm{H}(94 \mathrm{~A})$ & 4869 & 7450 & 5970 & 73 \\
\hline H(94B) & 4801 & 6544 & 5759 & 73 \\
\hline $\mathrm{H}(95 \mathrm{~A})$ & 5084 & 7345 & 5082 & 54 \\
\hline H(95B) & 5818 & 7825 & 5294 & 54 \\
\hline $\mathrm{H}(96 \mathrm{~A})$ & 6479 & 5953 & 5018 & 49 \\
\hline $\mathrm{H}(96 \mathrm{~B})$ & 7167 & 6657 & 4887 & 49 \\
\hline $\mathrm{H}(97 \mathrm{~A})$ & 1910 & 8179 & 6751 & 222 \\
\hline H(97B) & 1941 & 7138 & 6716 & 222 \\
\hline $\mathrm{H}(97 \mathrm{C})$ & 2611 & 7589 & 6309 & 222 \\
\hline H(99) & 4093 & 5847 & 6646 & 105 \\
\hline $\mathrm{H}(100)$ & 5584 & 5444 & 7054 & 100 \\
\hline $\mathrm{H}(101)$ & 5853 & 6428 & 7485 & 94 \\
\hline $\mathrm{H}(102)$ & 4590 & 7854 & 7583 & 80 \\
\hline H(103) & 3076 & 8383 & 7227 & 63 \\
\hline H(109) & 3948 & 3385 & 8891 & 208 \\
\hline $\mathrm{H}(111)$ & 4866 & 3697 & 7553 & 97 \\
\hline $\mathrm{H}(112)$ & 5734 & 2459 & 7428 & 167 \\
\hline
\end{tabular}




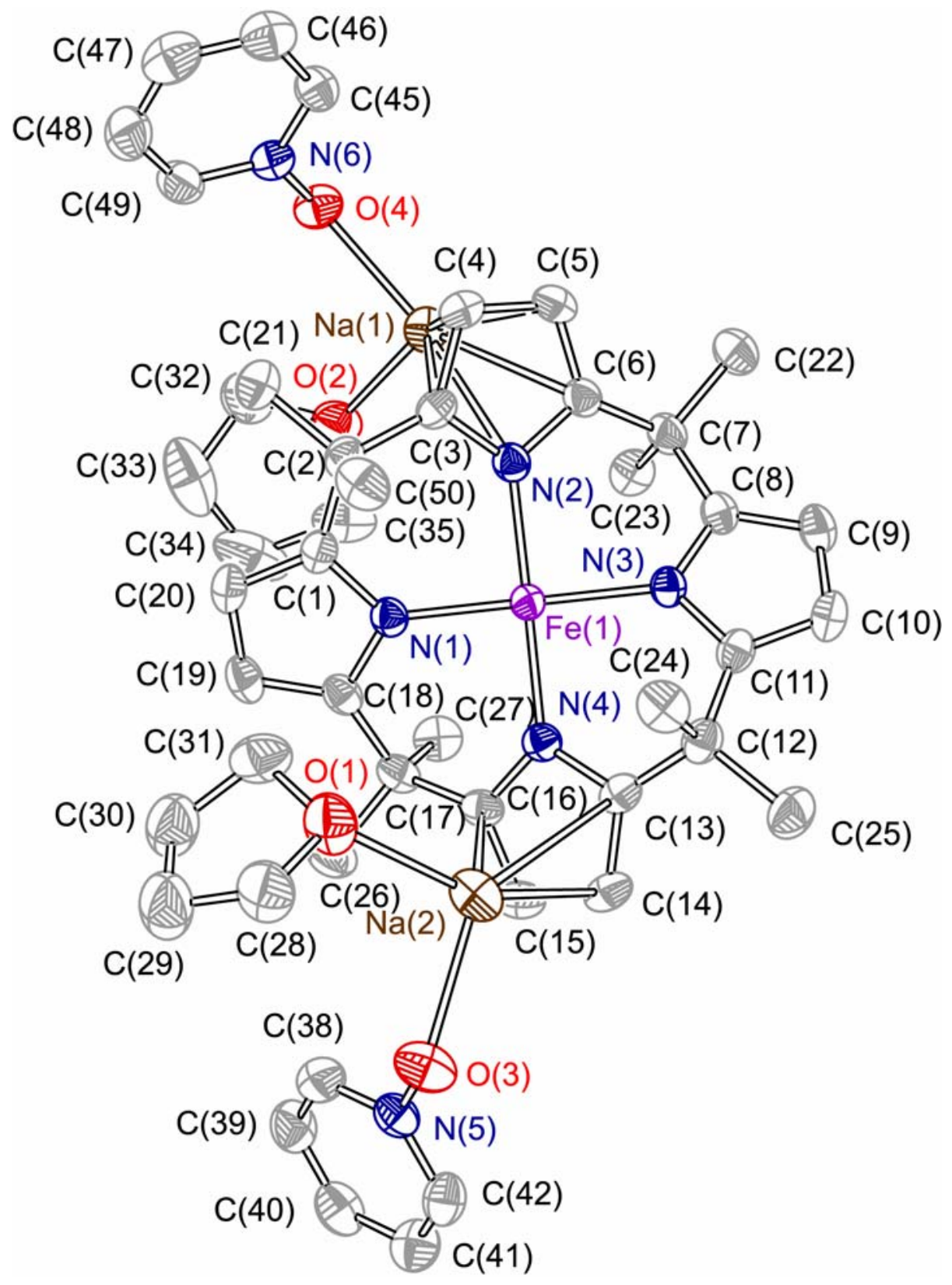

Figure S5. Atom labeling scheme for $[(\mathrm{THF}) \mathrm{Na}(\mathrm{Opy})]_{2}\left[\mathrm{LFe}^{\mathrm{II}}\right]$. 
Table S14. Crystal data and structure refinement for $[(\mathrm{THF}) \mathrm{Na}(\mathrm{Opy})]_{2}\left[\mathrm{LFe}^{\mathrm{II}}\right]$.

\begin{tabular}{|c|c|}
\hline Empirical formula & $\mathrm{C}_{50} \mathrm{H}_{58} \mathrm{FeN}_{6} \mathrm{Na}_{2} \mathrm{O}_{6}$ \\
\hline Formula weight & 908.85 \\
\hline$T(\mathrm{~K})$ & $183(2)$ \\
\hline$\lambda(\AA)$ & 0.71073 \\
\hline Crystal system & Monoclinic \\
\hline Space group & $P 2_{1} / \mathrm{c}$ \\
\hline$a(\AA)$ & $20.4824(13)$ \\
\hline$b(\AA)$ & $11.6675(7)$ \\
\hline$c(\AA)$ & 19.2151(12) \\
\hline$\alpha\left(^{\circ}\right)$ & 90 \\
\hline$\beta\left({ }^{\circ}\right)$ & $102.4720(10)^{\circ}$ \\
\hline$\gamma\left({ }^{\circ}\right)$ & 90 \\
\hline$V / \AA^{3}$ & $4483.6(5)$ \\
\hline$Z$ & 4 \\
\hline$\rho_{\text {calcd }}\left(\mathrm{g} / \mathrm{cm}^{3}\right)$ & 1.346 \\
\hline Absorption coefficient $\left(\mathrm{mm}^{-1}\right)$ & 0.409 \\
\hline$F(000)$ & 1920 \\
\hline Crystal size $\left(\mathrm{mm}^{3}\right)$ & $0.27 \times 0.27 \times 0.25$ \\
\hline$\theta$ range for data collection & 2.19 to $23.28^{\circ}$ \\
\hline Index ranges & $-21 \leq h \leq 22,-12 \leq k \leq 8,-21 \leq \ell \leq 21$ \\
\hline No. of coll. reflns & 17385 \\
\hline No. of ind. reflns $\left(\mathrm{R}_{\text {int }}\right)$ & $6414(0.0501)$ \\
\hline Completeness to $\theta=23.30^{\circ}$ & $99.4 \%$ \\
\hline Absorption correction & None \\
\hline Refinement method & Full-matrix least-squares on $F^{2}$ \\
\hline Data / restraints / parameters & 6414 / 0 / 764 \\
\hline $\mathrm{GOF}^{a}$ on $F^{2}$ & 1.064 \\
\hline$R 1,{ }^{b} \mathrm{w} R 2^{c}(\mathrm{I}>2 \sigma)$ & $0.0345,0.0840$ \\
\hline$R 1,{ }^{b} \mathrm{w} R 2^{c}$ (all data) & $0.0419,0.0876$ \\
\hline Extinction coefficient & not used \\
\hline Largest diff. peak and hole (e. $\left.\AA^{-3}\right)$ & 0.247 and -0.315 \\
\hline
\end{tabular}

${ }^{a} \mathrm{GOF}=\left(\sum w\left(F_{\mathrm{o}}{ }^{2}-F_{\mathrm{c}}{ }^{2}\right)^{2} /(n-p)\right)^{1 / 2}$ where $n$ is the number of data and $p$ is the number of parameters refined. ${ }^{b} R 1=\Sigma|| F_{\mathrm{o}}-\left|F_{\mathrm{c}} \|\right| \Sigma\left|F_{\mathrm{o}}\right| .{ }^{c} \mathrm{w} R 2=\left(\Sigma\left(w\left(F_{\mathrm{o}}{ }^{2}-\right.\right.\right.$ $\left.\left.\left.F_{\mathrm{c}}^{2}\right)^{2}\right) / \Sigma\left(w\left(F_{\mathrm{o}}^{2}\right)^{2}\right)\right)^{1 / 2}$. 
Table S15. Atomic coordinates $\left(\times 10^{4}\right)$ and equivalent isotropic displacement parameters $\left(\AA^{2} \times 10^{3}\right)$ for $[(\mathrm{THF}) \mathrm{Na}(\mathrm{Opy})]_{2}\left[\mathrm{LFe}^{\mathrm{II}}\right]$.

\begin{tabular}{|c|c|c|c|c|}
\hline Atom & $\mathrm{x}$ & $\mathrm{y}$ & $\mathrm{z}$ & $\mathrm{U}_{\mathrm{eq}}$ \\
\hline $\mathrm{Fe}(1)$ & $2508(1)$ & $6022(1)$ & $2442(1)$ & $25(1)$ \\
\hline $\mathrm{N}(1)$ & 2691(1) & $4482(1)$ & $2190(1)$ & $28(1)$ \\
\hline $\mathrm{N}(2)$ & $1564(1)$ & $5759(1)$ & 2071(1) & $28(1)$ \\
\hline $\mathrm{N}(3)$ & $2325(1)$ & $7587(1)$ & $2687(1)$ & $27(1)$ \\
\hline $\mathrm{N}(4)$ & $3452(1)$ & $6252(1)$ & $2840(1)$ & $28(1)$ \\
\hline $\mathrm{N}(5)$ & $5913(1)$ & $4536(2)$ & $4175(1)$ & $34(1)$ \\
\hline $\mathrm{N}(6)$ & $-390(1)$ & $2964(2)$ & $584(1)$ & $32(1)$ \\
\hline $\mathrm{Na}(1)$ & $700(1)$ & $5076(1)$ & $664(1)$ & $38(1)$ \\
\hline $\mathrm{Na}(2)$ & $4360(1)$ & $5108(1)$ & $4178(1)$ & $44(1)$ \\
\hline $\mathrm{C}(1)$ & $2268(1)$ & $3556(2)$ & $2147(1)$ & $30(1)$ \\
\hline $\mathrm{C}(2)$ & $1605(1)$ & $3645(2)$ & $2376(1)$ & $33(1)$ \\
\hline $\mathrm{C}(3)$ & $1233(1)$ & $4746(2)$ & $2133(1)$ & $29(1)$ \\
\hline $\mathrm{C}(4)$ & $552(1)$ & $4951(2)$ & 1992(1) & $34(1)$ \\
\hline$C(5)$ & $459(1)$ & $6129(2)$ & $1824(1)$ & $34(1)$ \\
\hline $\mathrm{C}(6)$ & 1084(1) & $6602(2)$ & 1873(1) & $30(1)$ \\
\hline$C(7)$ & $1295(1)$ & $7795(2)$ & $1713(1)$ & $35(1)$ \\
\hline $\mathrm{C}(8)$ & 1804(1) & $8266(2)$ & $2339(1)$ & $31(1)$ \\
\hline $\mathrm{C}(9)$ & $1854(1)$ & $9331(2)$ & $2653(1)$ & $39(1)$ \\
\hline$C(10)$ & $2424(1)$ & $9314(2)$ & $3215(1)$ & $39(1)$ \\
\hline $\mathrm{C}(11)$ & $2703(1)$ & $8239(2)$ & $3224(1)$ & $31(1)$ \\
\hline$C(12)$ & $3287(1)$ & $7710(2)$ & $3747(1)$ & $33(1)$ \\
\hline$C(13)$ & $3726(1)$ & $7035(2)$ & $3357(1)$ & $30(1)$ \\
\hline$C(14)$ & $4413(1)$ & $7023(2)$ & $3444(1)$ & $36(1)$ \\
\hline$C(15)$ & $4569(1)$ & $6199(2)$ & 2961(1) & $37(1)$ \\
\hline$C(16)$ & $3972(1)$ & $5747(2)$ & $2599(1)$ & $31(1)$ \\
\hline$C(17)$ & $3828(1)$ & $4933(2)$ & $1967(1)$ & $34(1)$ \\
\hline$C(18)$ & $3274(1)$ & $4104(2)$ & $2015(1)$ & $31(1)$ \\
\hline$C(19)$ & $3215(1)$ & $2951(2)$ & $1864(1)$ & $38(1)$ \\
\hline$C(20)$ & $2578(1)$ & $2604(2)$ & $1954(1)$ & $36(1)$ \\
\hline$C(21)$ & $1162(2)$ & $2627(2)$ & $2079(2)$ & $54(1)$ \\
\hline$C(22)$ & $684(1)$ & $8586(2)$ & $1547(2)$ & $49(1)$ \\
\hline$C(23)$ & $1607(1)$ & $7762(2)$ & $1050(1)$ & $41(1)$ \\
\hline$C(24)$ & $3018(1)$ & $6914(2)$ & $4260(1)$ & $43(1)$ \\
\hline$C(25)$ & $3700(2)$ & $8656(3)$ & $4190(2)$ & $50(1)$ \\
\hline$C(26)$ & $4462(1)$ & $4259(3)$ & $1921(2)$ & $48(1)$ \\
\hline$C(27)$ & $3634(1)$ & $5654(3)$ & $1281(1)$ & $43(1)$ \\
\hline$C(28)$ & $4306(2)$ & $2507(3)$ & $4744(2)$ & $59(1)$ \\
\hline
\end{tabular}




\begin{tabular}{rrrrr}
$\mathrm{C}(29)$ & $4495(2)$ & $1578(3)$ & $4294(2)$ & $80(1)$ \\
$\mathrm{C}(30)$ & $4064(2)$ & $1770(3)$ & $3553(2)$ & $65(1)$ \\
$\mathrm{C}(31)$ & $3567(1)$ & $2643(3)$ & $3673(1)$ & $53(1)$ \\
$\mathrm{C}(32)$ & $1299(2)$ & $3290(3)$ & $-370(2)$ & $61(1)$ \\
$\mathrm{C}(33)$ & $1904(2)$ & $2579(3)$ & $-44(2)$ & $81(1)$ \\
$\mathrm{C}(34)$ & $2452(2)$ & $3444(4)$ & $48(2)$ & $85(1)$ \\
$\mathrm{C}(35)$ & $2138(1)$ & $4504(3)$ & $253(2)$ & $59(1)$ \\
$\mathrm{C}(38)$ & $5795(1)$ & $3822(2)$ & $3616(1)$ & $45(1)$ \\
$\mathrm{C}(39)$ & $6233(1)$ & $3733(2)$ & $3173(1)$ & $49(1)$ \\
$\mathrm{C}(40)$ & $6806(1)$ & $4369(2)$ & $3303(1)$ & $47(1)$ \\
$\mathrm{C}(41)$ & $6922(1)$ & $5095(2)$ & $3880(1)$ & $47(1)$ \\
$\mathrm{C}(42)$ & $6473(1)$ & $5172(2)$ & $4311(1)$ & $41(1)$ \\
$\mathrm{C}(45)$ & $-886(1)$ & $3294(2)$ & $895(1)$ & $38(1)$ \\
$\mathrm{C}(46)$ & $-1178(1)$ & $2533(2)$ & $1269(1)$ & $48(1)$ \\
$\mathrm{C}(47)$ & $-966(1)$ & $1424(3)$ & $1342(1)$ & $52(1)$ \\
$\mathrm{C}(48)$ & $-463(1)$ & $1096(2)$ & $1020(2)$ & $54(1)$ \\
$\mathrm{C}(49)$ & $-181(1)$ & $1871(2)$ & $637(1)$ & $43(1)$ \\
$\mathrm{C}(50)$ & $1740(1)$ & $3587(3)$ & $3195(1)$ & $45(1)$ \\
$\mathrm{O}(1)$ & $3909(1)$ & $3306(2)$ & $4266(1)$ & $55(1)$ \\
$\mathrm{O}(2)$ & $1425(1)$ & $4345(2)$ & $14(1)$ & $48(1)$ \\
$\mathrm{O}(3)$ & $5480(1)$ & $4611(2)$ & $4595(1)$ & $48(1)$ \\
$\mathrm{O}(4)$ & $-111(1)$ & $3710(1)$ & $214(1)$ & $41(1)$ \\
\hline
\end{tabular}

${ }^{a} \mathrm{U}_{\mathrm{eq}}$ is defined as one third of the trace of the orthogonalized $\mathrm{U}_{\mathrm{ij}}$ tensor. 
Table S16. Bond lengths (in $\AA$ ) for $[(\mathrm{THF}) \mathrm{Na}(\mathrm{Opy})]_{2}\left[\mathrm{LFe}^{\mathrm{II}}\right]$.

\begin{tabular}{|c|c|c|c|}
\hline Bond & Length & Bond & Length \\
\hline $\mathrm{Fe}(1)-\mathrm{N}(1)$ & $1.9185(17)$ & $\mathrm{C}(2)-\mathrm{C}(21)$ & $1.528(3)$ \\
\hline $\mathrm{Fe}(1)-\mathrm{N}(2)$ & $1.9353(17)$ & $C(2)-C(50)$ & $1.539(3)$ \\
\hline $\mathrm{Fe}(1)-\mathrm{N}(4)$ & $1.9371(17)$ & $C(3)-C(4)$ & $1.383(3)$ \\
\hline $\mathrm{Fe}(1)-\mathrm{N}(3)$ & $1.9430(16)$ & $C(4)-C(5)$ & $1.415(3)$ \\
\hline $\mathrm{N}(1)-\mathrm{C}(1)$ & $1.376(3)$ & $C(5)-C(6)$ & $1.377(3)$ \\
\hline N(1)-C(18) & $1.381(3)$ & $C(6)-C(7)$ & $1.509(3)$ \\
\hline $\mathrm{N}(2)-\mathrm{C}(3)$ & $1.381(3)$ & $C(7)-C(8)$ & $1.516(3)$ \\
\hline $\mathrm{N}(2)-\mathrm{C}(6)$ & $1.385(3)$ & $C(7)-C(22)$ & $1.532(3)$ \\
\hline $\mathrm{N}(2)-\mathrm{Na}(1)$ & $3.0007(18)$ & $C(7)-C(23)$ & $1.544(3)$ \\
\hline $\mathrm{N}(3)-\mathrm{C}(11)$ & $1.378(3)$ & $C(8)-C(9)$ & $1.376(3)$ \\
\hline $\mathrm{N}(3)-\mathrm{C}(8)$ & $1.379(3)$ & $\mathrm{C}(9)-\mathrm{C}(10)$ & $1.408(3)$ \\
\hline $\mathrm{N}(4)-\mathrm{C}(13)$ & $1.378(3)$ & $\mathrm{C}(10)-\mathrm{C}(11)$ & $1.377(3)$ \\
\hline $\mathrm{N}(4)-\mathrm{C}(16)$ & $1.382(3)$ & $\mathrm{C}(11)-\mathrm{C}(12)$ & $1.517(3)$ \\
\hline $\mathrm{N}(5)-\mathrm{O}(3)$ & $1.326(2)$ & $C(12)-C(13)$ & $1.512(3)$ \\
\hline $\mathrm{N}(5)-\mathrm{C}(38)$ & $1.340(3)$ & $C(12)-C(25)$ & $1.532(3)$ \\
\hline $\mathrm{N}(5)-\mathrm{C}(42)$ & $1.343(3)$ & $\mathrm{C}(12)-\mathrm{C}(24)$ & $1.539(3)$ \\
\hline $\mathrm{N}(6)-\mathrm{O}(4)$ & $1.327(2)$ & $C(13)-C(14)$ & $1.379(3)$ \\
\hline $\mathrm{N}(6)-\mathrm{C}(49)$ & $1.341(3)$ & $\mathrm{C}(14)-\mathrm{C}(15)$ & $1.419(3)$ \\
\hline $\mathrm{N}(6)-\mathrm{C}(45)$ & $1.341(3)$ & $C(15)-C(16)$ & $1.376(3)$ \\
\hline $\mathrm{Na}(1)-\mathrm{O}(2)$ & $2.3009(18)$ & $C(16)-C(17)$ & $1.518(3)$ \\
\hline $\mathrm{Na}(1)-\mathrm{O}\left(4^{\prime}\right)$ & $2.3302(18)$ & $\mathrm{C}(17)-\mathrm{C}(18)$ & $1.510(3)$ \\
\hline $\mathrm{Na}(1)-\mathrm{O}(4)$ & $2.3306(18)$ & $C(17)-C(26)$ & $1.537(3)$ \\
\hline $\mathrm{Na}(1)-\mathrm{C}(4)$ & $2.637(2)$ & $\mathrm{C}(17)-\mathrm{C}(27)$ & $1.543(3)$ \\
\hline $\mathrm{Na}(1)-\mathrm{C}(5)$ & $2.682(2)$ & $\mathrm{C}(18)-\mathrm{C}(19)$ & $1.376(3)$ \\
\hline $\mathrm{Na}(1)-\mathrm{C}(3)$ & $2.824(2)$ & $C(19)-C(20)$ & $1.413(3)$ \\
\hline $\mathrm{Na}(1)-\mathrm{C}(6)$ & $2.898(2)$ & $\mathrm{C}(28)-\mathrm{O}(1)$ & $1.433(3)$ \\
\hline $\mathrm{Na}(1)-\mathrm{Na}\left(1^{\prime}\right)$ & $3.4076(17)$ & $\mathrm{C}(28)-\mathrm{C}(29)$ & $1.489(5)$ \\
\hline $\mathrm{Na}(2)-\mathrm{O}(1)$ & $2.317(2)$ & $C(29)-C(30)$ & $1.521(4)$ \\
\hline $\mathrm{Na}(2)-\mathrm{O}\left(3^{\prime \prime}\right)$ & $2.3328(18)$ & $\mathrm{C}(30)-\mathrm{C}(31)$ & $1.493(4)$ \\
\hline $\mathrm{Na}(2)-\mathrm{O}(3)$ & $2.3331(18)$ & $\mathrm{C}(31)-\mathrm{O}(1)$ & $1.429(3)$ \\
\hline $\mathrm{Na}(2)-\mathrm{C}(14)$ & $2.657(2)$ & $\mathrm{C}(32)-\mathrm{O}(2)$ & $1.430(3)$ \\
\hline $\mathrm{Na}(2)-\mathrm{C}(15)$ & $2.775(2)$ & $\mathrm{C}(32)-\mathrm{C}(33)$ & $1.509(4)$ \\
\hline $\mathrm{Na}(2)-\mathrm{C}(13)$ & $2.888(2)$ & $C(33)-C(34)$ & $1.492(6)$ \\
\hline $\mathrm{Na}(2)-\mathrm{C}(16)$ & $3.058(2)$ & $\mathrm{C}(34)-\mathrm{C}(35)$ & $1.486(5)$ \\
\hline $\mathrm{Na}(2)-\mathrm{Na}\left(2^{\prime \prime}\right)$ & $3.6528(18)$ & $\mathrm{C}(35)-\mathrm{O}(2)$ & $1.445(3)$ \\
\hline $\mathrm{C}(1)-\mathrm{C}(20)$ & $1.369(3)$ & $\mathrm{C}(38)-\mathrm{C}(39)$ & $1.368(4)$ \\
\hline$C(1)-C(2)$ & $1.519(3)$ & $\mathrm{C}(39)-\mathrm{C}(40)$ & $1.365(4)$ \\
\hline$C(2)-C(3)$ & $1.515(3)$ & $\mathrm{C}(40)-\mathrm{C}(41)$ & $1.375(4)$ \\
\hline
\end{tabular}




\begin{tabular}{llrr}
$\mathrm{C}(41)-\mathrm{C}(42)$ & $1.367(3)$ & $\mathrm{C}(48)-\mathrm{C}(49)$ & $1.370(4)$ \\
$\mathrm{C}(45)-\mathrm{C}(46)$ & $1.361(3)$ & $\mathrm{O}(3)-\mathrm{Na}\left(2^{\prime \prime}\right)$ & $2.3328(18)$ \\
$\mathrm{C}(46)-\mathrm{C}(47)$ & $1.362(4)$ & $\mathrm{O}(4)-\mathrm{Na}\left(1^{\prime}\right)$ & $2.3302(18)$ \\
$\mathrm{C}(47)-\mathrm{C}(48)$ & $1.367(4)$ & & \\
\hline
\end{tabular}


Table S17. Bond angles (in deg) for $[(\mathrm{THF}) \mathrm{Na}(\mathrm{Opy})]_{2}\left[\mathrm{LFe}^{\mathrm{II}}\right]$.

\begin{tabular}{|c|c|c|c|}
\hline Bond angle & Angle & Bond angle & Angle \\
\hline $\mathrm{N}(1)-\mathrm{Fe}(1)-\mathrm{N}(2)$ & $89.55(7)$ & $\mathrm{O}(2)-\mathrm{Na}(1)-\mathrm{C}(4)$ & $136.67(7)$ \\
\hline $\mathrm{N}(1)-\mathrm{Fe}(1)-\mathrm{N}(4)$ & $89.82(7)$ & $\mathrm{O}\left(4^{\prime}\right)-\mathrm{Na}(1)-\mathrm{C}(4)$ & $125.76(7)$ \\
\hline $\mathrm{N}(2)-\mathrm{Fe}(1)-\mathrm{N}(4)$ & $178.09(7)$ & $\mathrm{O}(4)-\mathrm{Na}(1)-\mathrm{C}(4)$ & $95.70(7)$ \\
\hline $\mathrm{N}(1)-\mathrm{Fe}(1)-\mathrm{N}(3)$ & $179.41(7)$ & $\mathrm{O}(2)-\mathrm{Na}(1)-\mathrm{C}(5)$ & $151.29(7)$ \\
\hline $\mathrm{N}(2)-\mathrm{Fe}(1)-\mathrm{N}(3)$ & $90.49(7)$ & $\mathrm{O}\left(4^{\prime}\right)-\mathrm{Na}(1)-\mathrm{C}(5)$ & $99.43(7)$ \\
\hline $\mathrm{N}(4)-\mathrm{Fe}(1)-\mathrm{N}(3)$ & $90.15(7)$ & $\mathrm{O}(4)-\mathrm{Na}(1)-\mathrm{C}(5)$ & $112.15(7)$ \\
\hline $\mathrm{C}(1)-\mathrm{N}(1)-\mathrm{C}(18)$ & 107.49(17) & $\mathrm{C}(4)-\mathrm{Na}(1)-\mathrm{C}(5)$ & $30.83(7)$ \\
\hline $\mathrm{C}(1)-\mathrm{N}(1)-\mathrm{Fe}(1)$ & $126.60(14)$ & $\mathrm{O}(2)-\mathrm{Na}(1)-\mathrm{C}(3)$ & $109.70(7)$ \\
\hline $\mathrm{C}(18)-\mathrm{N}(1)-\mathrm{Fe}(1)$ & $125.92(14)$ & $\mathrm{O}\left(4^{\prime}\right)-\mathrm{Na}(1)-\mathrm{C}(3)$ & $146.57(7)$ \\
\hline $\mathrm{C}(3)-\mathrm{N}(2)-\mathrm{C}(6)$ & $107.35(17)$ & $\mathrm{O}(4)-\mathrm{Na}(1)-\mathrm{C}(3)$ & $111.60(6)$ \\
\hline $\mathrm{C}(3)-\mathrm{N}(2)-\mathrm{Fe}(1)$ & $125.05(14)$ & $\mathrm{C}(4)-\mathrm{Na}(1)-\mathrm{C}(3)$ & $29.07(6)$ \\
\hline $\mathrm{C}(6)-\mathrm{N}(2)-\mathrm{Fe}(1)$ & $125.62(14)$ & $\mathrm{C}(5)-\mathrm{Na}(1)-\mathrm{C}(3)$ & $48.14(7)$ \\
\hline $\mathrm{C}(3)-\mathrm{N}(2)-\mathrm{Na}(1)$ & $69.26(10)$ & $\mathrm{O}(2)-\mathrm{Na}(1)-\mathrm{C}(6)$ & $124.62(7)$ \\
\hline $\mathrm{C}(6)-\mathrm{N}(2)-\mathrm{Na}(1)$ & $72.30(11)$ & $\mathrm{O}\left(4^{\prime}\right)-\mathrm{Na}(1)-\mathrm{C}(6)$ & $102.44(6)$ \\
\hline $\mathrm{Fe}(1)-\mathrm{N}(2)-\mathrm{Na}(1)$ & $136.21(7)$ & $\mathrm{O}(4)-\mathrm{Na}(1)-\mathrm{C}(6)$ & $139.98(7)$ \\
\hline $\mathrm{C}(11)-\mathrm{N}(3)-\mathrm{C}(8)$ & $107.27(17)$ & $\mathrm{C}(4)-\mathrm{Na}(1)-\mathrm{C}(6)$ & $47.64(7)$ \\
\hline $\mathrm{C}(11)-\mathrm{N}(3)-\mathrm{Fe}(1)$ & $126.43(14)$ & $\mathrm{C}(5)-\mathrm{Na}(1)-\mathrm{C}(6)$ & $28.25(6)$ \\
\hline $\mathrm{C}(8)-\mathrm{N}(3)-\mathrm{Fe}(1)$ & $126.26(13)$ & $\mathrm{C}(3)-\mathrm{Na}(1)-\mathrm{C}(6)$ & $45.82(6)$ \\
\hline$C(13)-N(4)-C(16)$ & $107.48(17)$ & $\mathrm{O}(2)-\mathrm{Na}(1)-\mathrm{N}(2)$ & $105.27(6)$ \\
\hline $\mathrm{C}(13)-\mathrm{N}(4)-\mathrm{Fe}(1)$ & $126.43(14)$ & $\mathrm{O}\left(4^{\prime}\right)-\mathrm{Na}(1)-\mathrm{N}(2)$ & $127.10(6)$ \\
\hline $\mathrm{C}(16)-\mathrm{N}(4)-\mathrm{Fe}(1)$ & $125.73(13)$ & $\mathrm{O}(4)-\mathrm{Na}(1)-\mathrm{N}(2)$ & $138.63(6)$ \\
\hline $\mathrm{O}(3)-\mathrm{N}(5)-\mathrm{C}(38)$ & $120.0(2)$ & $\mathrm{C}(4)-\mathrm{Na}(1)-\mathrm{N}(2)$ & $46.53(6)$ \\
\hline $\mathrm{O}(3)-\mathrm{N}(5)-\mathrm{C}(42)$ & $120.00(19)$ & $\mathrm{C}(5)-\mathrm{Na}(1)-\mathrm{N}(2)$ & $46.26(6)$ \\
\hline $\mathrm{C}(38)-\mathrm{N}(5)-\mathrm{C}(42)$ & $120.0(2)$ & $\mathrm{C}(3)-\mathrm{Na}(1)-\mathrm{N}(2)$ & $27.21(5)$ \\
\hline $\mathrm{O}(4)-\mathrm{N}(6)-\mathrm{C}(49)$ & $119.80(18)$ & $\mathrm{C}(6)-\mathrm{Na}(1)-\mathrm{N}(2)$ & $27.09(5)$ \\
\hline $\mathrm{O}(4)-\mathrm{N}(6)-\mathrm{C}(45)$ & $120.27(19)$ & $\mathrm{O}(2)-\mathrm{Na}(1)-\mathrm{Na}\left(1^{\prime}\right)$ & $96.40(5)$ \\
\hline $\mathrm{C}(49)-\mathrm{N}(6)-\mathrm{C}(45)$ & $119.9(2)$ & $\mathrm{O}\left(4^{\prime}\right)-\mathrm{Na}(1)-\mathrm{Na}\left(1^{\prime}\right)$ & $43.02(4)$ \\
\hline $\mathrm{O}(2)-\mathrm{Na}(1)-\mathrm{O}\left(4^{\prime}\right)$ & $97.22(6)$ & $\mathrm{O}(4)-\mathrm{Na}(1)-\mathrm{Na}\left(1^{\prime}\right)$ & $43.01(4)$ \\
\hline $\mathrm{O}(2)-\mathrm{Na}(1)-\mathrm{O}(4)$ & $92.14(7)$ & $\mathrm{C}(4)-\mathrm{Na}(1)-\mathrm{Na}\left(1^{\prime}\right)$ & $117.88(6)$ \\
\hline $\mathrm{O}\left(4^{\prime}\right)-\mathrm{Na}(1)-\mathrm{O}(4)$ & $86.04(6)$ & $\mathrm{C}(5)-\mathrm{Na}(1)-\mathrm{Na}\left(1^{\prime}\right)$ & $111.71(6)$ \\
\hline $\mathrm{C}(3)-\mathrm{Na}(1)-\mathrm{Na}\left(1^{\prime}\right)$ & $145.33(6)$ & $C(4)-C(3)-C(2)$ & $128.8(2)$ \\
\hline $\mathrm{C}(6)-\mathrm{Na}(1)-\mathrm{Na}\left(1^{\prime}\right)$ & $132.15(6)$ & $\mathrm{N}(2)-\mathrm{C}(3)-\mathrm{Na}(1)$ & $83.53(11)$ \\
\hline $\mathrm{N}(2)-\mathrm{Na}(1)-\mathrm{Na}\left(1^{\prime}\right)$ & $157.77(5)$ & $\mathrm{C}(4)-\mathrm{C}(3)-\mathrm{Na}(1)$ & $67.95(12)$ \\
\hline $\mathrm{O}(1)-\mathrm{Na}(2)-\mathrm{O}\left(3^{\prime \prime}\right)$ & $91.45(7)$ & $\mathrm{C}(2)-\mathrm{C}(3)-\mathrm{Na}(1)$ & $118.93(13)$ \\
\hline $\mathrm{O}(1)-\mathrm{Na}(2)-\mathrm{O}(3)$ & $97.34(7)$ & $C(3)-C(4)-C(5)$ & $107.1(2)$ \\
\hline $\mathrm{O}\left(3^{\prime \prime}\right)-\mathrm{Na}(2)-\mathrm{O}(3)$ & $76.95(6)$ & $\mathrm{C}(3)-\mathrm{C}(4)-\mathrm{Na}(1)$ & $82.98(13)$ \\
\hline $\mathrm{O}(1)-\mathrm{Na}(2)-\mathrm{C}(14)$ & $149.79(8)$ & $\mathrm{C}(5)-\mathrm{C}(4)-\mathrm{Na}(1)$ & $76.34(13)$ \\
\hline $\mathrm{O}\left(3^{\prime \prime}\right)-\mathrm{Na}(2)-\mathrm{C}(14)$ & $113.91(8)$ & $\mathrm{C}(6)-\mathrm{C}(5)-\mathrm{C}(4)$ & 107.23(19) \\
\hline $\mathrm{O}(3)-\mathrm{Na}(2)-\mathrm{C}(14)$ & $103.99(7)$ & $\mathrm{C}(6)-\mathrm{C}(5)-\mathrm{Na}(1)$ & $84.59(13)$ \\
\hline
\end{tabular}




\begin{tabular}{|c|c|}
\hline $\mathrm{O}(1)-\mathrm{Na}(2)-\mathrm{C}(15)$ & $127.90(7)$ \\
\hline $\mathrm{O}\left(3^{\prime \prime}\right)-\mathrm{Na}(2)-\mathrm{C}(15)$ & $140.65(8)$ \\
\hline $\mathrm{O}(3)-\mathrm{Na}(2)-\mathrm{C}(15)$ & $94.86(7)$ \\
\hline $\mathrm{C}(14)-\mathrm{Na}(2)-\mathrm{C}(15)$ & $30.18(7)$ \\
\hline $\mathrm{O}(1)-\mathrm{Na}(2)-\mathrm{C}(13)$ & $127.36(7)$ \\
\hline $\mathrm{O}\left(3^{\prime \prime}\right)-\mathrm{Na}(2)-\mathrm{C}(13)$ & $113.01(7)$ \\
\hline $\mathrm{O}(3)-\mathrm{Na}(2)-\mathrm{C}(13)$ & $132.32(7)$ \\
\hline $\mathrm{C}(14)-\mathrm{Na}(2)-\mathrm{C}(13)$ & $28.41(6)$ \\
\hline $\mathrm{C}(15)-\mathrm{Na}(2)-\mathrm{C}(13)$ & $46.81(7)$ \\
\hline $\mathrm{O}(1)-\mathrm{Na}(2)-\mathrm{C}(16)$ & $105.73(7)$ \\
\hline $\mathrm{O}\left(3^{\prime \prime}\right)-\mathrm{Na}(2)-\mathrm{C}(16)$ & $156.68(7)$ \\
\hline $\mathrm{O}(3)-\mathrm{Na}(2)-\mathrm{C}(16)$ & $115.30(6)$ \\
\hline $\mathrm{C}(14)-\mathrm{Na}(2)-\mathrm{C}(16)$ & $45.57(7)$ \\
\hline $\mathrm{C}(15)-\mathrm{Na}(2)-\mathrm{C}(16)$ & $26.72(6)$ \\
\hline $\mathrm{C}(13)-\mathrm{Na}(2)-\mathrm{C}(16)$ & $43.84(6)$ \\
\hline $\mathrm{O}(1)-\mathrm{Na}(2)-\mathrm{Na}\left(2^{\prime \prime}\right)$ & $95.60(5)$ \\
\hline $\mathrm{O}\left(3^{\prime \prime}\right)-\mathrm{Na}(2)-\mathrm{Na}\left(2^{\prime \prime}\right)$ & $38.48(4)$ \\
\hline $\mathrm{O}(3)-\mathrm{Na}(2)-\mathrm{Na}\left(2^{\prime \prime}\right)$ & $38.47(4)$ \\
\hline $\mathrm{C}(14)-\mathrm{Na}(2)-\mathrm{Na}\left(2^{\prime \prime}\right)$ & $114.41(6)$ \\
\hline $\mathrm{C}(15)-\mathrm{Na}(2)-\mathrm{Na}\left(2^{\prime \prime}\right)$ & $123.23(6)$ \\
\hline $\mathrm{C}(13)-\mathrm{Na}(2)-\mathrm{Na}\left(2^{\prime \prime}\right)$ & $132.82(6)$ \\
\hline $\mathrm{C}(16)-\mathrm{Na}(2)-\mathrm{Na}\left(2^{\prime \prime}\right)$ & $149.25(6)$ \\
\hline $\mathrm{C}(20)-\mathrm{C}(1)-\mathrm{N}(1)$ & 109.31(19) \\
\hline$C(20)-C(1)-C(2)$ & $129.0(2)$ \\
\hline $\mathrm{N}(1)-\mathrm{C}(1)-\mathrm{C}(2)$ & $121.29(18)$ \\
\hline$C(3)-C(2)-C(1)$ & $112.93(17)$ \\
\hline$C(3)-C(2)-C(21)$ & $108.99(19)$ \\
\hline$C(1)-C(2)-C(21)$ & $109.5(2)$ \\
\hline$C(3)-C(2)-C(50)$ & $108.63(18)$ \\
\hline$C(1)-C(2)-C(50)$ & $108.60(18)$ \\
\hline$C(21)-C(2)-C(50)$ & $108.0(2)$ \\
\hline $\mathrm{N}(2)-\mathrm{C}(3)-\mathrm{C}(4)$ & $109.16(19)$ \\
\hline $\mathrm{N}(2)-\mathrm{C}(3)-\mathrm{C}(2)$ & $121.93(18)$ \\
\hline$C(13)-C(14)-C(15)$ & $107.2(2)$ \\
\hline $\mathrm{C}(13)-\mathrm{C}(14)-\mathrm{Na}(2)$ & $85.15(13)$ \\
\hline $\mathrm{C}(15)-\mathrm{C}(14)-\mathrm{Na}(2)$ & $79.51(14)$ \\
\hline$C(16)-C(15)-C(14)$ & $106.9(2)$ \\
\hline $\mathrm{C}(16)-\mathrm{C}(15)-\mathrm{Na}(2)$ & $88.21(13)$ \\
\hline $\mathrm{C}(14)-\mathrm{C}(15)-\mathrm{Na}(2)$ & $70.32(12)$ \\
\hline$C(15)-C(16)-N(4)$ & 109.37(19) \\
\hline$C(15)-C(16)-C(17)$ & 129.97(19) \\
\hline $\mathrm{N}(4)-\mathrm{C}(16)-\mathrm{C}(17)$ & $120.29(17)$ \\
\hline $\mathrm{C}(15)-\mathrm{C}(16)-\mathrm{Na}(2)$ & $65.07(12)$ \\
\hline
\end{tabular}

\begin{tabular}{|c|c|}
\hline $\mathrm{C}(4)-\mathrm{C}(5)-\mathrm{Na}(1)$ & \\
\hline$C(5)-C(6)-N(2)$ & $109.18(19)$ \\
\hline$C(5)-C(6)-C(7)$ & $7(19)$ \\
\hline $\mathrm{N}(2)-\mathrm{C}(6)-\mathrm{C}(7)$ & $19.54(18)$ \\
\hline $\mathrm{C}(5)-\mathrm{C}(6)-\mathrm{Na}(1)$ & $67.16(12)$ \\
\hline $\mathrm{N}(2)-\mathrm{C}(6)-\mathrm{Na}(1)$ & 80.6 \\
\hline $\mathrm{C}(7)-\mathrm{C}(6)-\mathrm{Na}(1)$ & $116.29(13)$ \\
\hline$C(6)-C(7)-C(8)$ & 110. \\
\hline $\mathrm{C}(6)-\mathrm{C}(7)-\mathrm{C}(22)$ & 109. \\
\hline $\mathrm{C}(8)-\mathrm{C}(7)-\mathrm{C}(22)$ & 109. \\
\hline $\mathrm{C}(6)-\mathrm{C}(7)-\mathrm{C}(23)$ & 109 \\
\hline $\mathrm{C}(8)-\mathrm{C}(7)-\mathrm{C}(23)$ & 109. \\
\hline $\mathrm{C}(22)-\mathrm{C}(7)-\mathrm{C}(23)$ & \\
\hline $\mathrm{C}(9)-\mathrm{C}(8)-\mathrm{N}(3)$ & 109.2 \\
\hline $\mathrm{C}(9)-\mathrm{C}(8$ & 130 \\
\hline $\mathrm{N}(3)-\mathrm{C}(8$ & 120 \\
\hline $\mathrm{C}(8)-\mathrm{C}(9)-\mathrm{C}(10)$ & \\
\hline$C(11)-C(10)-C(9)$ & $10^{\prime}$ \\
\hline$C(10)-C(1)$ & 109.1 \\
\hline$C(10)-C(11)$ & 130 \\
\hline $\mathrm{N}(3)-\mathrm{C}(11)-\mathrm{C}(12)$ & \\
\hline$C(13)-C(12)-C(11)$ & 110.6 \\
\hline 13$)-C(12)-C(25)$ & (2) \\
\hline$(11)-C(12)-C(25)$ & \\
\hline 13$)-C(12)-C(24)$ & 109. \\
\hline$(11)-C(12)-C(24)$ & $109.23(19)$ \\
\hline$(25)-C(12)-C(24)$ & $1(2)$ \\
\hline $\mathrm{N}(4)-\mathrm{C}(13)-\mathrm{C}(14)$ & 109. \\
\hline $\mathrm{N}(4)-\mathrm{C}(13)-\mathrm{C}(12)$ & $120.61(18)$ \\
\hline$C(14)-C(13)-C(12)$ & $130.3(2)$ \\
\hline $\mathrm{N}(4)-\mathrm{C}(13)-\mathrm{Na}(2)$ & $86.74(12)$ \\
\hline$(14)-\mathrm{C}(13)-\mathrm{Na}(2)$ & (12) \\
\hline $\mathrm{C}(12)-\mathrm{C}(13)-\mathrm{Na}(2)$ & $112.36(12)$ \\
\hline $\mathrm{O}(2)-\mathrm{C}(32)-\mathrm{C}(33)$ & $102.6(3)$ \\
\hline $\mathrm{C}(34)-\mathrm{C}(33)-\mathrm{C}(32)$ & $101.9(3)$ \\
\hline$C(35)-C(34)-C(33)$ & $103.7(3)$ \\
\hline $\mathrm{O}(2)-\mathrm{C}(35)-\mathrm{C}(34)$ & $105.9(3)$ \\
\hline $\mathrm{N}(5)-\mathrm{C}(38)-\mathrm{C}(39)$ & $121.1(3)$ \\
\hline $\mathrm{C}(40)-\mathrm{C}(39)-\mathrm{C}(38)$ & $120.0(2)$ \\
\hline$C(39)-C(40)-C(41)$ & $118.3(2)$ \\
\hline$C(42)-C(41)-C(40)$ & $120.6(3)$ \\
\hline $\mathrm{N}(5)-\mathrm{C}(42)-\mathrm{C}(41)$ & $120.2(2)$ \\
\hline $\mathrm{N}(6)-\mathrm{C}(45)-\mathrm{C}(46)$ & $120.7(2)$ \\
\hline
\end{tabular}




$\begin{array}{rrrr}\mathrm{N}(4)-\mathrm{C}(16)-\mathrm{Na}(2) & 79.95(11) & \mathrm{C}(45)-\mathrm{C}(46)-\mathrm{C}(47) & 120.5(3) \\ \mathrm{C}(17)-\mathrm{C}(16)-\mathrm{Na}(2) & 127.17(14) & \mathrm{C}(46)-\mathrm{C}(47)-\mathrm{C}(48) & 118.3(3) \\ \mathrm{C}(18)-\mathrm{C}(17)-\mathrm{C}(16) & 11.76(17) & \mathrm{C}(47)-\mathrm{C}(48)-\mathrm{C}(49) & 120.3(3) \\ \mathrm{C}(18)-\mathrm{C}(17)-\mathrm{C}(26) & 109.4(2) & \mathrm{N}(6)-\mathrm{C}(49)-\mathrm{C}(48) & 120.3(2) \\ \mathrm{C}(16)-\mathrm{C}(17)-\mathrm{C}(26) & 110.27(19) & \mathrm{C}(31)-\mathrm{O}(1)-\mathrm{C}(28) & 105.8(2) \\ \mathrm{C}(18)-\mathrm{C}(17)-\mathrm{C}(27) & 110.03(19) & \mathrm{C}(31)-\mathrm{O}(1)-\mathrm{Na}(2) & 124.59(16) \\ \mathrm{C}(16)-\mathrm{C}(17)-\mathrm{C}(27) & 108.18(19) & \mathrm{C}(28)-\mathrm{O}(1)-\mathrm{Na}(2) & 117.17(18) \\ \mathrm{C}(26)-\mathrm{C}(17)-\mathrm{C}(27) & 107.1(2) & \mathrm{C}(32)-\mathrm{O}(2)-\mathrm{C}(35) & 109.4(2) \\ \mathrm{C}(19)-\mathrm{C}(18)-\mathrm{N}(1) & 108.87(19) & \mathrm{C}(32)-\mathrm{O}(2)-\mathrm{Na}(1) & 122.28(17) \\ \mathrm{C}(19)-\mathrm{C}(18)-\mathrm{C}(17) & 130.2(2) & \mathrm{C}(35)-\mathrm{O}(2)-\mathrm{Na}(1) & 120.38(15) \\ \mathrm{N}(1)-\mathrm{C}(18)-\mathrm{C}(17) & 120.74(18) & \mathrm{N}(5)-\mathrm{O}(3)-\mathrm{Na}\left(2^{\prime \prime}\right) & 131.17(13) \\ \mathrm{C}(18)-\mathrm{C}(19)-\mathrm{C}(20) & 107.1(2) & \mathrm{N}(5)-\mathrm{O}(3)-\mathrm{Na}(2) & 123.04(12) \\ \mathrm{C}(1)-\mathrm{C}(20)-\mathrm{C}(19) & 107.2(2) & \mathrm{Na}\left(2^{\prime \prime}\right)-\mathrm{O}(3)-\mathrm{Na}(2) & 103.05(6) \\ \mathrm{O}(1)-\mathrm{C}(28)-\mathrm{C}(29) & 106.5(2) & \mathrm{N}(6)-\mathrm{O}(4)-\mathrm{Na}\left(1^{\prime}\right) & 124.86(12) \\ \mathrm{C}(28)-\mathrm{C}(29)-\mathrm{C}(30) & 105.3(3) & \mathrm{N}(6)-\mathrm{O}(4)-\mathrm{Na}(1) & 127.16(12) \\ \mathrm{C}(31)-\mathrm{C}(30)-\mathrm{C}(29) & 103.5(3) & \mathrm{Na}\left(1^{\prime}\right)-\mathrm{O}(4)-\mathrm{Na}(1) & 93.96(6) \\ \mathrm{O}(1)-\mathrm{C}(31)-\mathrm{C}(30) & 105.0(2) & & \end{array}$

Primed labels refer to atoms generated by the following symmetry transformation: $-\mathrm{x},-\mathrm{y}+1,-\mathrm{z}$. Double-primed labels refer to: $-\mathrm{x}+1,-\mathrm{y}+1,-\mathrm{z}+1$ 
Table S18. Anisotropic displacement parameters $\left(\AA^{2} \times 10^{3}\right)$ for $[(\mathrm{THF}) \mathrm{Na}(\mathrm{Opy})]_{2}\left[\mathrm{LFe}^{\mathrm{II}}\right]$.

\begin{tabular}{|c|c|c|c|c|c|c|}
\hline Atom & $\mathrm{U}_{11}$ & $\mathrm{U}_{22}$ & $\mathrm{U}_{33}$ & $\mathrm{U}_{23}$ & $\mathrm{U}_{13}$ & $\mathrm{U}_{12}$ \\
\hline $\mathrm{Fe}(1)$ & $25(1)$ & $23(1)$ & $26(1)$ & $0(1)$ & $4(1)$ & $1(1)$ \\
\hline $\mathrm{N}(1)$ & $30(1)$ & $27(1)$ & $26(1)$ & $0(1)$ & $3(1)$ & $3(1)$ \\
\hline $\mathrm{N}(2)$ & $29(1)$ & $27(1)$ & $27(1)$ & $1(1)$ & $4(1)$ & $3(1)$ \\
\hline $\mathrm{N}(3)$ & $32(1)$ & $24(1)$ & $27(1)$ & $1(1)$ & $6(1)$ & $1(1)$ \\
\hline $\mathrm{N}(4)$ & $28(1)$ & $29(1)$ & $27(1)$ & $2(1)$ & $4(1)$ & $1(1)$ \\
\hline $\mathrm{N}(5)$ & $36(1)$ & $38(1)$ & $30(1)$ & $5(1)$ & $7(1)$ & $7(1)$ \\
\hline $\mathrm{N}(6)$ & $31(1)$ & $37(1)$ & $26(1)$ & $3(1)$ & $3(1)$ & $-3(1)$ \\
\hline $\mathrm{Na}(1)$ & $43(1)$ & $40(1)$ & $34(1)$ & $1(1)$ & $12(1)$ & $-1(1)$ \\
\hline $\mathrm{Na}(2)$ & $46(1)$ & $49(1)$ & $41(1)$ & 11(1) & $17(1)$ & $9(1)$ \\
\hline $\mathrm{C}(1)$ & $37(1)$ & $25(1)$ & $25(1)$ & $1(1)$ & $0(1)$ & $-1(1)$ \\
\hline$C(2)$ & $35(1)$ & $29(1)$ & $33(1)$ & $2(1)$ & $6(1)$ & $-3(1)$ \\
\hline $\mathrm{C}(3)$ & $31(1)$ & $32(1)$ & $23(1)$ & $-2(1)$ & $3(1)$ & $-5(1)$ \\
\hline$C(4)$ & $29(1)$ & $40(1)$ & $32(1)$ & $-2(1)$ & $6(1)$ & $-7(1)$ \\
\hline$C(5)$ & $27(1)$ & $41(1)$ & $32(1)$ & $0(1)$ & $5(1)$ & $7(1)$ \\
\hline$C(6)$ & $29(1)$ & $32(1)$ & $29(1)$ & $-1(1)$ & $2(1)$ & $4(1)$ \\
\hline$C(7)$ & $34(1)$ & $31(1)$ & $36(1)$ & $4(1)$ & $2(1)$ & $7(1)$ \\
\hline $\mathrm{C}(8)$ & $35(1)$ & $26(1)$ & $32(1)$ & $3(1)$ & $8(1)$ & $4(1)$ \\
\hline $\mathrm{C}(9)$ & $46(1)$ & $26(1)$ & $45(1)$ & $4(1)$ & $9(1)$ & $8(1)$ \\
\hline$C(10)$ & $51(2)$ & $26(1)$ & $41(1)$ & $-7(1)$ & $9(1)$ & $-4(1)$ \\
\hline $\mathrm{C}(11)$ & $37(1)$ & $28(1)$ & $28(1)$ & $-2(1)$ & $10(1)$ & $-4(1)$ \\
\hline$C(12)$ & $40(1)$ & $32(1)$ & $27(1)$ & $-2(1)$ & $4(1)$ & $-3(1)$ \\
\hline$C(13)$ & $33(1)$ & $29(1)$ & $26(1)$ & $6(1)$ & $1(1)$ & $-5(1)$ \\
\hline $\mathrm{C}(14)$ & $34(1)$ & $40(1)$ & $31(1)$ & $4(1)$ & $-2(1)$ & $-11(1)$ \\
\hline$C(15)$ & $27(1)$ & $49(2)$ & $35(1)$ & $8(1)$ & $7(1)$ & $-1(1)$ \\
\hline$C(16)$ & $27(1)$ & $35(1)$ & $28(1)$ & $7(1)$ & $4(1)$ & $2(1)$ \\
\hline$C(17)$ & $29(1)$ & $41(1)$ & $33(1)$ & $0(1)$ & $8(1)$ & $7(1)$ \\
\hline $\mathrm{C}(18)$ & $33(1)$ & $34(1)$ & $26(1)$ & $1(1)$ & $5(1)$ & $8(1)$ \\
\hline C(19) & $46(1)$ & $34(1)$ & $35(1)$ & $-5(1)$ & $10(1)$ & $12(1)$ \\
\hline$C(20)$ & $48(2)$ & $25(1)$ & $34(1)$ & $-3(1)$ & $7(1)$ & $1(1)$ \\
\hline $\mathrm{C}(21)$ & $47(2)$ & $33(2)$ & $79(2)$ & $1(1)$ & $9(2)$ & $-6(1)$ \\
\hline $\mathrm{C}(22)$ & $43(2)$ & $39(2)$ & $58(2)$ & $5(1)$ & $-1(1)$ & $12(1)$ \\
\hline$C(23)$ & $48(2)$ & $41(2)$ & $31(1)$ & $5(1)$ & $2(1)$ & $-1(1)$ \\
\hline$C(24)$ & $48(2)$ & $49(2)$ & $32(1)$ & $6(1)$ & $9(1)$ & $5(1)$ \\
\hline$C(25)$ & $54(2)$ & $47(2)$ & $43(2)$ & $-13(1)$ & $-2(1)$ & $-3(1)$ \\
\hline$C(26)$ & $35(1)$ & $58(2)$ & $53(2)$ & $-8(2)$ & $15(1)$ & $9(1)$ \\
\hline$C(27)$ & $44(2)$ & $51(2)$ & $34(1)$ & $3(1)$ & 11(1) & $-2(1)$ \\
\hline$C(28)$ & $71(2)$ & $53(2)$ & $45(2)$ & $10(1)$ & $-8(2)$ & $8(2)$ \\
\hline$C(29)$ & $94(3)$ & $51(2)$ & $75(2)$ & $-4(2)$ & $-23(2)$ & $13(2)$ \\
\hline
\end{tabular}




\begin{tabular}{rrrrrrr}
$\mathrm{C}(30)$ & $78(2)$ & $51(2)$ & $57(2)$ & $-6(2)$ & $-3(2)$ & $0(2)$ \\
$\mathrm{C}(31)$ & $47(2)$ & $65(2)$ & $42(2)$ & $11(1)$ & $-4(1)$ & $-6(1)$ \\
$\mathrm{C}(32)$ & $60(2)$ & $72(2)$ & $52(2)$ & $-19(2)$ & $15(2)$ & $7(2)$ \\
$\mathrm{C}(33)$ & $134(4)$ & $73(2)$ & $35(2)$ & $-2(2)$ & $16(2)$ & $46(2)$ \\
$\mathrm{C}(34)$ & $54(2)$ & $145(4)$ & $53(2)$ & $-6(2)$ & $3(2)$ & $35(2)$ \\
$\mathrm{C}(35)$ & $41(2)$ & $94(2)$ & $43(2)$ & $1(2)$ & $9(1)$ & $-1(2)$ \\
$\mathrm{C}(38)$ & $41(2)$ & $47(2)$ & $45(1)$ & $-6(1)$ & $4(1)$ & $-1(1)$ \\
$\mathrm{C}(39)$ & $57(2)$ & $49(2)$ & $40(1)$ & $-13(1)$ & $10(1)$ & $9(1)$ \\
$\mathrm{C}(40)$ & $54(2)$ & $51(2)$ & $42(1)$ & $4(1)$ & $21(1)$ & $12(1)$ \\
$\mathrm{C}(41)$ & $51(2)$ & $45(2)$ & $48(2)$ & $4(1)$ & $18(1)$ & $-6(1)$ \\
$\mathrm{C}(42)$ & $54(2)$ & $35(1)$ & $34(1)$ & $-2(1)$ & $12(1)$ & $-2(1)$ \\
$\mathrm{C}(45)$ & $40(1)$ & $39(2)$ & $35(1)$ & $-1(1)$ & $12(1)$ & $3(1)$ \\
$\mathrm{C}(46)$ & $47(2)$ & $60(2)$ & $40(1)$ & $0(1)$ & $19(1)$ & $-4(1)$ \\
$\mathrm{C}(47)$ & $54(2)$ & $58(2)$ & $43(1)$ & $16(1)$ & $8(1)$ & $-13(1)$ \\
$\mathrm{C}(48)$ & $55(2)$ & $38(2)$ & $63(2)$ & $14(1)$ & $2(1)$ & $4(1)$ \\
$\mathrm{C}(49)$ & $36(1)$ & $44(2)$ & $48(1)$ & $5(1)$ & $9(1)$ & $6(1)$ \\
$\mathrm{C}(50)$ & $47(2)$ & $47(2)$ & $42(1)$ & $15(1)$ & $15(1)$ & $8(1)$ \\
$\mathrm{O}(1)$ & $71(1)$ & $49(1)$ & $38(1)$ & $3(1)$ & $-5(1)$ & $12(1)$ \\
$\mathrm{O}(2)$ & $39(1)$ & $59(1)$ & $45(1)$ & $-8(1)$ & $8(1)$ & $5(1)$ \\
$\mathrm{O}(3)$ & $40(1)$ & $70(1)$ & $38(1)$ & $6(1)$ & $19(1)$ & $10(1)$ \\
$\mathrm{O}(4)$ & $42(1)$ & $44(1)$ & $39(1)$ & $9(1)$ & $14(1)$ & $-7(1)$ \\
\hline
\end{tabular}

The anisotropic displacement factor exponent takes the form: $-2 \pi^{2} h^{2} a^{* 2} U_{11}+\ldots+$ $2 \mathrm{hka} * \mathrm{~b} * \mathrm{U}_{12}$ ] 
Table S19. Hydrogen coordinates $\left(\times 10^{4}\right)$ and isotropic displacement parameters $\left(\AA^{2} \times 10^{3}\right)$ for $[(\mathrm{THF}) \mathrm{Na}(\mathrm{Opy})]_{2}\left[\mathrm{LFe}^{\mathrm{II}}\right]$.

\begin{tabular}{|c|c|c|c|c|}
\hline Atom & $\mathrm{x}$ & $\mathrm{y}$ & $\mathrm{z}$ & $\mathrm{U}(\mathrm{eq})$ \\
\hline $\mathrm{H}(1)$ & $224(11)$ & $4430(20)$ & $2012(11)$ & $32(6)$ \\
\hline $\mathrm{H}(2)$ & $49(11)$ & $6517(19)$ & $1700(11)$ & $32(6)$ \\
\hline $\mathrm{H}(3)$ & $1564(11)$ & $9950(20)$ & $2493(12)$ & $37(6)$ \\
\hline $\mathrm{H}(4)$ & $2586(11)$ & $9920(20)$ & $3515(12)$ & $36(6)$ \\
\hline $\mathrm{H}(5)$ & $4711(10)$ & $7486(18)$ & $3744(11)$ & $25(5)$ \\
\hline $\mathrm{H}(6)$ & 4999(13) & $6020(20)$ & $2886(12)$ & $45(7)$ \\
\hline $\mathrm{H}(7)$ & $3543(11)$ & $2490(20)$ & $1720(12)$ & $42(6)$ \\
\hline $\mathrm{H}(8)$ & $2406(10)$ & $1880(20)$ & $1922(11)$ & $33(6)$ \\
\hline $\mathrm{H}(9)$ & $358(13)$ & $8280(20)$ & $1137(14)$ & $49(7)$ \\
\hline $\mathrm{H}(10)$ & $810(13)$ & $9370(30)$ & $1423(14)$ & $63(8)$ \\
\hline $\mathrm{H}(11)$ & $483(12)$ & $8660(20)$ & $1939(14)$ & $48(7)$ \\
\hline $\mathrm{H}(12)$ & $1732(12)$ & $8530(30)$ & $906(14)$ & $56(7)$ \\
\hline $\mathrm{H}(13)$ & $1299(13)$ & $7460(20)$ & $640(14)$ & $49(7)$ \\
\hline $\mathrm{H}(14)$ & $2005(13)$ & $7290(20)$ & $1133(13)$ & $46(7)$ \\
\hline $\mathrm{H}(15)$ & $3871(13)$ & $9150(20)$ & $3905(14)$ & $52(8)$ \\
\hline $\mathrm{H}(16)$ & $3425(13)$ & $9120(20)$ & $4431(14)$ & $53(8)$ \\
\hline $\mathrm{H}(17)$ & $4053(13)$ & $8290(20)$ & $4569(14)$ & $55(7)$ \\
\hline $\mathrm{H}(18)$ & $2724(12)$ & $7350(20)$ & $4525(12)$ & $45(7)$ \\
\hline H(19) & $3393(13)$ & $6610(20)$ & $4597(14)$ & $55(7)$ \\
\hline $\mathrm{H}(20)$ & $2753(11)$ & $6270(20)$ & $4015(12)$ & $37(6)$ \\
\hline $\mathrm{H}(21)$ & $4813(14)$ & $4760(20)$ & $1898(13)$ & $51(7)$ \\
\hline $\mathrm{H}(22)$ & $4601(12)$ & $3780(20)$ & $2341(14)$ & $47(7)$ \\
\hline $\mathrm{H}(23)$ & $4385(13)$ & $3770(20)$ & $1500(15)$ & $57(8)$ \\
\hline $\mathrm{H}(24)$ & $3969(12)$ & $6190(20)$ & $1243(12)$ & $41(7)$ \\
\hline $\mathrm{H}(25)$ & $3251(14)$ & $6100(20)$ & $1267(14)$ & $55(8)$ \\
\hline $\mathrm{H}(26)$ & $3547(12)$ & $5140(20)$ & $854(14)$ & $51(7)$ \\
\hline $\mathrm{H}(27)$ & $1079(13)$ & $2630(20)$ & $1552(16)$ & $60(8)$ \\
\hline $\mathrm{H}(28)$ & $1387(15)$ & $1950(30)$ & $2256(16)$ & $74(9)$ \\
\hline $\mathrm{H}(29)$ & $754(16)$ & $2630(30)$ & $2242(15)$ & $69(9)$ \\
\hline $\mathrm{H}(30)$ & $1964(12)$ & $2840(20)$ & $3362(13)$ & $51(7)$ \\
\hline $\mathrm{H}(31)$ & $1310(12)$ & $3689(19)$ & $3367(12)$ & $38(6)$ \\
\hline $\mathrm{H}(32)$ & $2018(12)$ & $4200(20)$ & $3409(12)$ & $37(6)$ \\
\hline $\mathrm{H}(33)$ & $6515(12)$ & $5670(20)$ & $4699(14)$ & $50(7)$ \\
\hline $\mathrm{H}(34)$ & $7325(14)$ & $5520(20)$ & $4004(14)$ & $59(8)$ \\
\hline $\mathrm{H}(35)$ & $7111(13)$ & $4310(20)$ & $3018(14)$ & $53(7)$ \\
\hline $\mathrm{H}(36)$ & $6109(13)$ & $3230(20)$ & $2804(15)$ & $63(8)$ \\
\hline $\mathrm{H}(37)$ & $5394(13)$ & $3420(20)$ & $3553(13)$ & $49(7)$ \\
\hline
\end{tabular}




\begin{tabular}{rrrrr}
$\mathrm{H}(38)$ & $-1000(11)$ & $4060(20)$ & $821(11)$ & $33(6)$ \\
$\mathrm{H}(39)$ & $-1516(14)$ & $2750(20)$ & $1449(15)$ & $67(9)$ \\
$\mathrm{H}(40)$ & $-1150(14)$ & $900(20)$ & $1621(15)$ & $64(8)$ \\
$\mathrm{H}(41)$ & $-303(14)$ & $350(30)$ & $1032(15)$ & $72(9)$ \\
$\mathrm{H}(42)$ & $166(12)$ & $1700(20)$ & $383(13)$ & $46(7)$ \\
$\mathrm{H}(43)$ & $1305(14)$ & $3460(30)$ & $-893(17)$ & $72(9)$ \\
$\mathrm{H}(44)$ & $862(17)$ & $3020(30)$ & $-346(17)$ & $86(11)$ \\
$\mathrm{H}(45)$ & $1953(15)$ & $1960(30)$ & $-333(18)$ & $82(10)$ \\
$\mathrm{H}(46)$ & $1815(17)$ & $2290(30)$ & $360(20)$ & $94(11)$ \\
$\mathrm{H}(47)$ & $2571(18)$ & $3610(30)$ & $-380(20)$ & $100(13)$ \\
$\mathrm{H}(48)$ & $2864(19)$ & $3260(30)$ & $390(20)$ & $102(12)$ \\
$\mathrm{H}(49)$ & $2248(15)$ & $5150(30)$ & $-5(17)$ & $79(10)$ \\
$\mathrm{H}(50)$ & $2239(11)$ & $4590(20)$ & $773(14)$ & $47(7)$ \\
$\mathrm{H}(51)$ & $4663(16)$ & $2870(30)$ & $5014(17)$ & $76(10)$ \\
$\mathrm{H}(52)$ & $4041(17)$ & $2160(30)$ & $5087(19)$ & $96(11)$ \\
$\mathrm{H}(53)$ & $3183(15)$ & $2200(30)$ & $3807(16)$ & $77(9)$ \\
$\mathrm{H}(54)$ & $3411(12)$ & $3150(20)$ & $3289(13)$ & $46(7)$ \\
$\mathrm{H}(55)$ & $3838(15)$ & $1080(30)$ & $3324(16)$ & $76(9)$ \\
$\mathrm{H}(56)$ & $4360(19)$ & $2140(30)$ & $3210(20)$ & $118(14)$ \\
$\mathrm{H}(57)$ & $4449(17)$ & $900(30)$ & $4480(19)$ & $92(12)$ \\
$\mathrm{H}(58)$ & $4980(30)$ & $1640(50)$ & $4320(30)$ & $180(20)$ \\
\hline & & & &
\end{tabular}



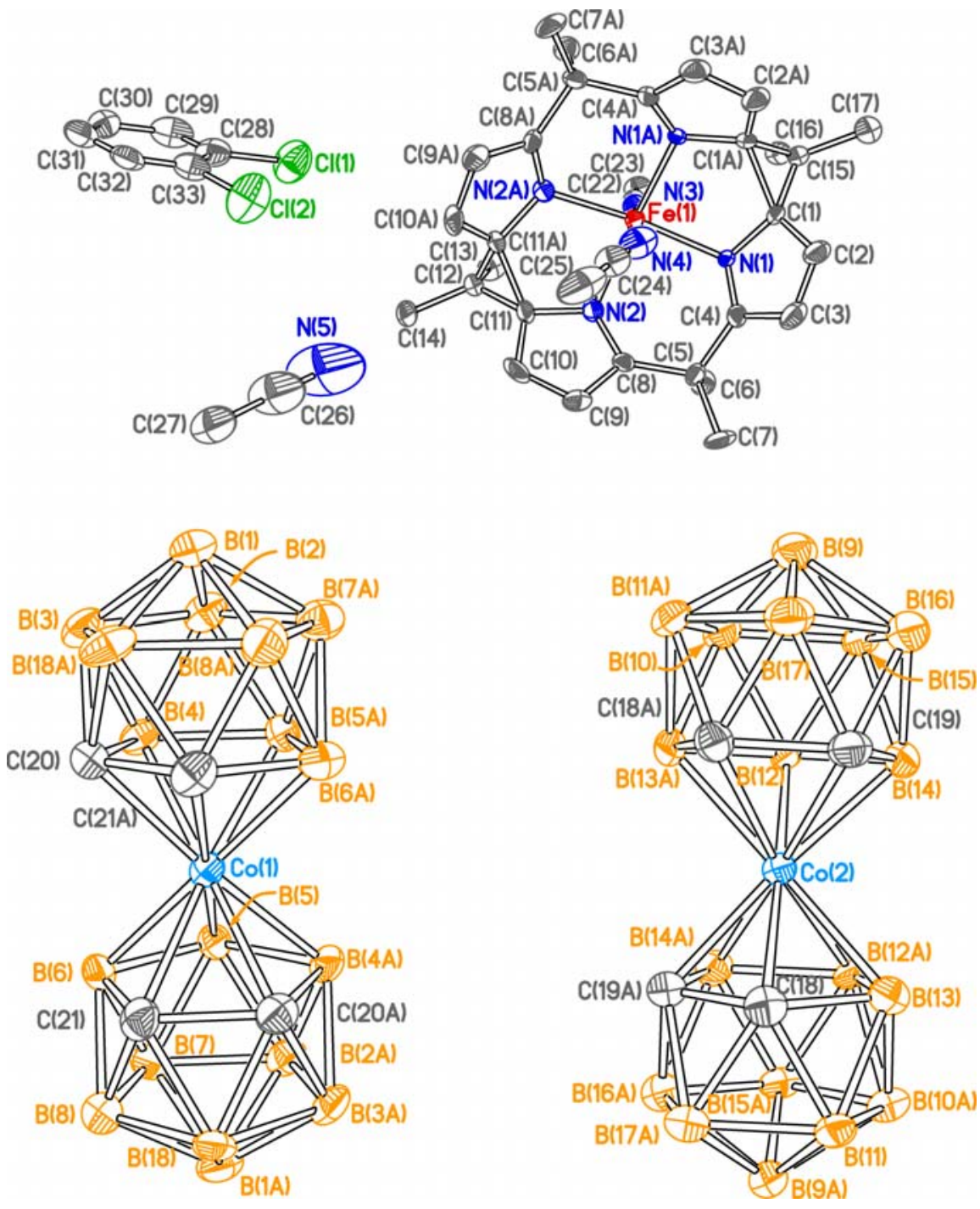

Figure S6. Atom labeling scheme for $\left[\mathrm{L}^{\Delta \Lambda} \mathrm{Fe}^{\mathrm{II}}\left(\mathrm{NCCH}_{3}\right)\right] \quad\left[\left(\mathrm{C}_{2} \mathrm{~B}_{9} \mathrm{H}_{11}\right)_{2} \mathrm{Co}\right]_{2}$ - $2 \mathrm{CH}_{3} \mathrm{CN} \cdot 2 o-\mathrm{C}_{6} \mathrm{H}_{4} \mathrm{Cl}_{2}$. For clarity, each cobalticarborane has been separated from the main structure (top, including the dication and the solvents) and labeled individually (bottom). 
Table S20. Crystal data and structure refinement for $\left[\mathrm{L}^{\Delta \Delta} \mathrm{Fe}^{\mathrm{II}}\left(\mathrm{NCCH}_{3}\right)\right]$ $\left[\left(\mathrm{C}_{2} \mathrm{~B}_{9} \mathrm{H}_{11}\right)_{2} \mathrm{Co}\right]_{2} \cdot 2 \mathrm{CH}_{3} \mathrm{CN} \cdot 2 o-\mathrm{C}_{6} \mathrm{H}_{4} \mathrm{Cl}_{2}$

\begin{tabular}{|c|c|}
\hline Empirical formula & $\mathrm{C}_{54} \mathrm{H}_{93} \mathrm{~B}_{36} \mathrm{Cl}_{4} \mathrm{Co}_{2} \mathrm{FeN}_{7}$ \\
\hline Formula weight & 1545.02 \\
\hline$T(\mathrm{~K})$ & $100(2)$ \\
\hline$\lambda(\AA)$ & 0.71073 \\
\hline Crystal system & Orthorhombic \\
\hline Space group & Abm2 \\
\hline$a(\AA)$ & $23.443(2)$ \\
\hline$b(\AA)$ & $25.146(3)$ \\
\hline$c(\AA)$ & $13.2883(13)$ \\
\hline$\alpha\left(^{\circ}\right)$ & 90 \\
\hline$\beta\left(^{\circ}\right)$ & 90 \\
\hline$\gamma\left({ }^{\circ}\right)$ & 90 \\
\hline$V / \AA^{3}$ & $7833.6(14)$ \\
\hline Z & 4 \\
\hline$\rho_{\text {calcd }}\left(\mathrm{g} / \mathrm{cm}^{3}\right)$ & 1.310 \\
\hline Absorption coefficient $\left(\mathrm{mm}^{-1}\right)$ & 0.780 \\
\hline$F(000)$ & 3176 \\
\hline Crystal size $\left(\mathrm{mm}^{3}\right)$ & $0.12 \times 0.11 \times 0.09$ \\
\hline$\theta$ range for data collection & 0.87 to $23.31^{\circ}$. \\
\hline Index ranges & $-26 \leq h \leq 25,-27 \leq k \leq 27,-9 \leq \ell \leq 14$ \\
\hline No. of coll. reflns & 19041 \\
\hline No. of ind. reflns $\left(\mathrm{R}_{\mathrm{int}}\right)$ & $4642(0.0705)$ \\
\hline Completeness to $\theta=23.30^{\circ}$ & $99.5 \%$ \\
\hline Absorption correction & None \\
\hline Refinement method & Full-matrix least-squares on $\mathrm{F}^{2}$ \\
\hline Data / restraints / parameters & 4642 / 23 / 592 \\
\hline $\mathrm{GOF}^{a}$ on $F^{2}$ & 1.112 \\
\hline$R 1{ }^{b} \mathrm{w} R 2^{c}(\mathrm{I}>2 \sigma)$ & $0.0533,0.1385$ \\
\hline$R 1,{ }^{b} \mathrm{w} R 2^{c}$ (all data) & $0.0556,0.1411$ \\
\hline Extinction coefficient & Not used \\
\hline Absolute structure parameter & $0.66(3)$ \\
\hline Largest diff. peak and hole (e. $\left.\AA^{-3}\right)$ & 0.927 and -0.758 \\
\hline
\end{tabular}

${ }^{a} \mathrm{GOF}=\left(\sum w\left(F_{\mathrm{o}}{ }^{2}-F_{\mathrm{c}}{ }^{2}\right)^{2} /(n-p)\right)^{1 / 2}$ where $n$ is the number of data and $p$ is the number of parameters refined. ${ }^{b} R 1=\Sigma|| F_{\mathrm{o}}-\left|F_{\mathrm{c}}\right||/ \Sigma| F_{\mathrm{o}} \mid .{ }^{c} \mathrm{w} R 2=\left(\Sigma\left(w\left(F_{\mathrm{o}}{ }^{2}-\right.\right.\right.$ $\left.\left.\left.F_{\mathrm{c}}^{2}\right)^{2}\right) / \Sigma\left(w\left(F_{\mathrm{o}}^{2}\right)^{2}\right)\right)^{1 / 2}$. 
Table S21. Atomic coordinates $\left(\times 10^{4}\right)$ and equivalent isotropic displacement parameters $\left(\AA^{2} \times 10^{3}\right)$ for $\left[\mathrm{L}^{\Delta \Delta} \mathrm{Fe}^{\mathrm{II}}\left(\mathrm{NCCH}_{3}\right)\right]\left[\left(\mathrm{C}_{2} \mathrm{~B}_{9} \mathrm{H}_{11}\right)_{2} \mathrm{Co}\right]_{2} \cdot 2 \mathrm{CH}_{3} \mathrm{CN} \cdot 2 o-$ $\mathrm{C}_{6} \mathrm{H}_{4} \mathrm{Cl}_{2}$

\begin{tabular}{|c|c|c|c|c|}
\hline Atom & $\mathrm{x}$ & $\mathrm{y}$ & $\mathrm{z}$ & $\mathrm{U}_{\mathrm{eq}}$ \\
\hline $\mathrm{Fe}(1)$ & $8123(1)$ & 7500 & $9118(1)$ & $17(1)$ \\
\hline $\operatorname{Co}(1)$ & 5000 & 10000 & $915(1)$ & $20(1)$ \\
\hline $\operatorname{Co}(2)$ & 0 & 10000 & $1429(1)$ & $18(1)$ \\
\hline $\mathrm{N}(1)$ & $7871(2)$ & $6962(2)$ & $10259(4)$ & $16(1)$ \\
\hline $\mathrm{N}(2)$ & $7757(2)$ & $6967(2)$ & $8068(4)$ & $20(1)$ \\
\hline $\mathrm{N}(3)$ & 8999(3) & 7500 & $8974(6)$ & $23(2)$ \\
\hline $\mathrm{N}(4)$ & $6753(5)$ & 7500 & $9369(9)$ & $51(3)$ \\
\hline $\mathrm{N}(5)$ & $6349(9)$ & 7500 & $4500(30)$ & $224(18)$ \\
\hline $\mathrm{C}(1)$ & $7717(3)$ & $7180(2)$ & $11226(5)$ & $21(2)$ \\
\hline $\mathrm{C}(2)$ & $7265(3)$ & $6855(3)$ & $11638(6)$ & $32(2)$ \\
\hline$C(3)$ & $7175(3)$ & $6468(3)$ & $10989(6)$ & $33(2)$ \\
\hline $\mathrm{C}(4)$ & $7557(3)$ & $6549(2)$ & $10137(5)$ & $21(1)$ \\
\hline$C(5)$ & $7589(2)$ & $6209(2)$ & $9207(6)$ & $22(1)$ \\
\hline$C(6)$ & $8204(2)$ & $5994(2)$ & $9115(7)$ & $29(1)$ \\
\hline$C(7)$ & $7178(3)$ & $5729(2)$ & $9282(7)$ & $33(2)$ \\
\hline$C(8)$ & $7453(3)$ & $6534(2)$ & $8291(5)$ & $20(1)$ \\
\hline $\mathrm{C}(9)$ & $7006(3)$ & $6458(3)$ & $7554(6)$ & $33(2)$ \\
\hline$C(10)$ & $7027(3)$ & $6850(3)$ & $6872(6)$ & $32(2)$ \\
\hline$C(11)$ & $7512(3)$ & $7191(2)$ & $7163(5)$ & $23(1)$ \\
\hline$C(12)$ & $7876(5)$ & 7500 & $6433(8)$ & $30(2)$ \\
\hline$C(13)$ & $8523(4)$ & 7500 & $6572(8)$ & $33(3)$ \\
\hline$C(14)$ & $7697(6)$ & 7500 & $5340(8)$ & $43(3)$ \\
\hline$C(15)$ & $8158(4)$ & 7500 & $11816(8)$ & $29(2)$ \\
\hline$C(16)$ & $8774(4)$ & 7500 & $11478(8)$ & $28(2)$ \\
\hline$C(17)$ & $8109(5)$ & 7500 & $12943(9)$ & $37(3)$ \\
\hline$C(18)$ & $105(3)$ & $10624(3)$ & $451(6)$ & $24(2)$ \\
\hline$C(19)$ & $537(3)$ & $9441(2)$ & $857(7)$ & $26(2)$ \\
\hline$C(20)$ & $4524(3)$ & $9400(3)$ & $329(6)$ & $25(2)$ \\
\hline $\mathrm{C}(21)$ & $4818(3)$ & $10603(3)$ & $-81(6)$ & $26(2)$ \\
\hline$C(22)$ & $9496(4)$ & 7500 & $8891(7)$ & $23(2)$ \\
\hline $\mathrm{C}(23)$ & $10094(4)$ & 7500 & $8877(9)$ & $32(2)$ \\
\hline $\mathrm{C}(24)$ & $6323(4)$ & 7500 & $9041(10)$ & $35(2)$ \\
\hline$C(25)$ & $5734(6)$ & 7500 & $8679(12)$ & $67(5)$ \\
\hline$C(26)$ & $6098(7)$ & 7500 & $3820(19)$ & $100(8)$ \\
\hline $\mathrm{C}(27)$ & $5731(7)$ & 7500 & $2979(13)$ & $68(4)$ \\
\hline $\mathrm{C}(28)$ & $7758(3)$ & $9331(3)$ & $3446(7)$ & $45(2)$ \\
\hline C(29) & $8053(3)$ & $9609(3)$ & $2783(8)$ & $54(2)$ \\
\hline
\end{tabular}




\begin{tabular}{rrrrr}
$\mathrm{C}(30)$ & $7758(3)$ & $9891(3)$ & $1988(7)$ & $38(2)$ \\
$\mathrm{C}(31)$ & $7204(4)$ & $9891(3)$ & $1942(8)$ & $51(2)$ \\
$\mathrm{C}(32)$ & $6874(4)$ & $9578(3)$ & $2676(7)$ & $47(2)$ \\
$\mathrm{C}(33)$ & $7150(3)$ & $9311(3)$ & $3390(7)$ & $42(2)$ \\
$\mathrm{B}(1)$ & $5011(3)$ & $8446(3)$ & $916(9)$ & $31(2)$ \\
$\mathrm{B}(2)$ & $4821(3)$ & $8833(3)$ & $1989(7)$ & $28(2)$ \\
$\mathrm{B}(3)$ & $4371(3)$ & $8825(3)$ & $939(8)$ & $28(2)$ \\
$\mathrm{B}(4)$ & $4492(4)$ & $9434(3)$ & $1614(6)$ & $23(2)$ \\
$\mathrm{B}(5)$ & $4774(3)$ & $10562(3)$ & $2014(7)$ & $25(2)$ \\
$\mathrm{B}(6)$ & $4347(3)$ & $10567(3)$ & $890(8)$ & $25(2)$ \\
$\mathrm{B}(7)$ & $4455(4)$ & $11170(3)$ & $1555(7)$ & $29(2)$ \\
$\mathrm{B}(8)$ & $4490(3)$ & $11177(3)$ & $229(7)$ & $30(2)$ \\
$\mathrm{B}(9)$ & $159(3)$ & $8456(3)$ & $1481(8)$ & $31(2)$ \\
$\mathrm{B}(10)$ & $-418(3)$ & $8783(3)$ & $2095(7)$ & $24(2)$ \\
$\mathrm{B}(11)$ & $383(3)$ & $11232(3)$ & $754(7)$ & $26(2)$ \\
$\mathrm{B}(12)$ & $-163(3)$ & $9423(3)$ & $2527(7)$ & $21(2)$ \\
$\mathrm{B}(13)$ & $583(3)$ & $10629(3)$ & $1380(8)$ & $23(2)$ \\
$\mathrm{B}(14)$ & $575(3)$ & $9487(3)$ & $2146(7)$ & $24(2)$ \\
$\mathrm{B}(15)$ & $299(3)$ & $8858(3)$ & $2536(7)$ & $25(2)$ \\
$\mathrm{B}(16)$ & $758(3)$ & $8888(3)$ & $1479(8)$ & $32(2)$ \\
$\mathrm{B}(17)$ & $342(3)$ & $8825(3)$ & $396(8)$ & $31(2)$ \\
$\mathrm{B}(18)$ & $5212(4)$ & $11191(3)$ & $-169(7)$ & $29(2)$ \\
$\mathrm{Cl}(1)$ & $8116(1)$ & $8995(1)$ & $4394(2)$ & $71(1)$ \\
$\mathrm{Cl}(2)$ & $6777(1)$ & $8929(1)$ & $4220(3)$ & $74(1)$ \\
\hline
\end{tabular}

${ }^{a} \mathrm{U}_{\mathrm{eq}}$ is defined as one third of the trace of the orthogonalized $\mathrm{U}_{\mathrm{ij}}$ tensor. 
Table S22. Bond lengths ( $\AA$ ) for $\left[\mathrm{L}^{\Delta \Lambda} \mathrm{Fe}^{\mathrm{II}}\left(\mathrm{NCCH}_{3}\right)\right]\left[\left(\mathrm{C}_{2} \mathrm{~B}_{9} \mathrm{H}_{11}\right)_{2} \mathrm{Co}\right]_{2}$ $\cdot 2 \mathrm{CH}_{3} \mathrm{CN} \cdot 2 o-\mathrm{C}_{6} \mathrm{H}_{4} \mathrm{Cl}_{2}$

\begin{tabular}{|c|c|c|c|}
\hline Bond & Length & Bond & Length \\
\hline $\mathrm{Fe}(1)-\mathrm{N}(3)$ & $2.063(7)$ & $\mathrm{C}(9)-\mathrm{C}(10)$ & $1.340(11)$ \\
\hline $\mathrm{Fe}(1)-\mathrm{N}(2)$ & $2.116(5)$ & $\mathrm{C}(10)-\mathrm{C}(11)$ & $1.474(9)$ \\
\hline $\mathrm{Fe}(1)-\mathrm{N}\left(2^{\prime}\right)$ & $2.116(5)$ & $C(11)-C(12)$ & $1.509(12)$ \\
\hline $\mathrm{Fe}(1)-\mathrm{N}(1)$ & $2.116(5)$ & $\mathrm{C}(11)-\mathrm{C}\left(11^{\prime}\right)$ & $1.555(12)$ \\
\hline $\mathrm{Fe}(1)-\mathrm{N}\left(1^{\prime}\right)$ & $2.116(5)$ & $C(12)-C\left(11^{\prime}\right)$ & $1.509(11)$ \\
\hline $\mathrm{Co}(1)-\mathrm{C}\left(20^{\prime \prime}\right)$ & $2.033(7)$ & $\mathrm{C}(12)-\mathrm{C}(14)$ & $1.513(15)$ \\
\hline $\mathrm{Co}(1)-\mathrm{C}(20)$ & $2.033(7)$ & $C(12)-C(13)$ & $1.526(15)$ \\
\hline $\mathrm{Co}(1)-\mathrm{C}\left(21^{\prime \prime}\right)$ & $2.058(7)$ & $C(15)-C(17)$ & $1.501(15)$ \\
\hline $\mathrm{Co}(1)-\mathrm{C}(21)$ & $2.058(7)$ & $C(15)-C(16)$ & $1.511(15)$ \\
\hline $\mathrm{Co}(1)-\mathrm{B}\left(4^{\prime \prime}\right)$ & $2.075(8)$ & $\mathrm{C}(15)-\mathrm{C}\left(1^{\prime}\right)$ & $1.527(11)$ \\
\hline $\mathrm{Co}(1)-\mathrm{B}(4)$ & $2.075(8)$ & $\mathrm{C}(18)-\mathrm{C}\left(19^{\prime \prime \prime}\right)$ & $1.609(9)$ \\
\hline $\mathrm{Co}(1)-\mathrm{B}\left(6^{\prime \prime}\right)$ & $2.092(7)$ & $\mathrm{C}(18)-\mathrm{B}(13)$ & $1.667(12)$ \\
\hline $\mathrm{Co}(1)-\mathrm{B}(6)$ & $2.092(7)$ & $\mathrm{C}(18)-\mathrm{B}(11)$ & $1.709(10)$ \\
\hline $\mathrm{Co}(1)-\mathrm{B}(5)$ & $2.101(8)$ & $\mathrm{C}(18)-\mathrm{B}\left(17^{\prime \prime \prime}\right)$ & $1.739(10)$ \\
\hline $\mathrm{Co}(1)-\mathrm{B}\left(5^{\prime \prime}\right)$ & $2.101(8)$ & $\mathrm{C}(19)-\mathrm{C}\left(18^{\prime \prime \prime}\right)$ & $1.609(9)$ \\
\hline $\mathrm{Co}(2)-\mathrm{C}\left(19^{\prime \prime \prime}\right)$ & $2.034(6)$ & $\mathrm{C}(19)-\mathrm{B}(16)$ & $1.698(11)$ \\
\hline $\mathrm{Co}(2)-\mathrm{C}(19)$ & $2.034(6)$ & $\mathrm{C}(19)-\mathrm{B}(14)$ & $1.719(12)$ \\
\hline $\mathrm{Co}(2)-\mathrm{C}\left(18^{\prime \prime \prime}\right)$ & $2.053(7)$ & C(19)-B(17) & $1.730(10)$ \\
\hline $\mathrm{Co}(2)-\mathrm{C}(18)$ & $2.053(7)$ & $C(20)-C\left(21^{\prime \prime}\right)$ & $1.635(9)$ \\
\hline $\mathrm{Co}(2)-\mathrm{B}\left(12^{\prime \prime \prime}\right)$ & $2.092(8)$ & $\mathrm{C}(20)-\mathrm{B}(3)$ & $1.694(10)$ \\
\hline $\mathrm{Co}(2)-\mathrm{B}(12)$ & $2.092(8)$ & $\mathrm{C}(20)-\mathrm{B}(4)$ & $1.712(10)$ \\
\hline $\mathrm{Co}(2)-\mathrm{B}\left(13^{\prime \prime \prime}\right)$ & $2.092(7)$ & $\mathrm{C}(20)-\mathrm{B}\left(18^{\prime \prime}\right)$ & $1.740(10)$ \\
\hline $\mathrm{Co}(2)-\mathrm{B}(13)$ & $2.092(7)$ & $\mathrm{C}(21)-\mathrm{C}\left(20^{\prime \prime}\right)$ & $1.635(9)$ \\
\hline $\mathrm{Co}(2)-\mathrm{B}(14)$ & $2.095(8)$ & $\mathrm{C}(21)-\mathrm{B}(8)$ & $1.687(11)$ \\
\hline $\mathrm{Co}(2)-\mathrm{B}\left(14^{\prime \prime \prime}\right)$ & $2.095(8)$ & $\mathrm{C}(21)-\mathrm{B}(6)$ & $1.701(12)$ \\
\hline $\mathrm{N}(1)-\mathrm{C}(4)$ & $1.285(8)$ & $\mathrm{C}(21)-\mathrm{B}(18)$ & $1.748(10)$ \\
\hline $\mathrm{N}(1)-\mathrm{C}(1)$ & $1.444(8)$ & $\mathrm{C}(22)-\mathrm{C}(23)$ & $1.403(12)$ \\
\hline $\mathrm{N}(2)-\mathrm{C}(8)$ & $1.336(8)$ & $\mathrm{C}(24)-\mathrm{C}(25)$ & $1.460(17)$ \\
\hline $\mathrm{N}(2)-\mathrm{C}(11)$ & $1.446(8)$ & $\mathrm{C}(26)-\mathrm{C}(27)$ & $1.41(3)$ \\
\hline $\mathrm{N}(3)-\mathrm{C}(22)$ & $1.171(11)$ & $\mathrm{C}(28)-\mathrm{C}(29)$ & $1.321(13)$ \\
\hline $\mathrm{N}(4)-\mathrm{C}(24)$ & $1.100(13)$ & $\mathrm{C}(28)-\mathrm{C}(33)$ & $1.428(11)$ \\
\hline $\mathrm{N}(5)-\mathrm{C}(26)$ & $1.08(3)$ & $\mathrm{C}(28)-\mathrm{Cl}(1)$ & $1.734(9)$ \\
\hline$C(1)-C(2)$ & $1.445(9)$ & $\mathrm{C}(29)-\mathrm{C}(30)$ & $1.447(13)$ \\
\hline $\mathrm{C}(1)-\mathrm{C}(15)$ & $1.527(11)$ & $\mathrm{C}(30)-\mathrm{C}(31)$ & $1.300(12)$ \\
\hline $\mathrm{C}(1)-\mathrm{C}\left(1^{\prime}\right)$ & $1.609(11)$ & $\mathrm{C}(31)-\mathrm{C}(32)$ & $1.473(13)$ \\
\hline$C(2)-C(3)$ & $1.316(11)$ & $\mathrm{C}(32)-\mathrm{C}(33)$ & $1.330(12)$ \\
\hline$C(3)-C(4)$ & $1.459(10)$ & $\mathrm{C}(33)-\mathrm{Cl}(2)$ & $1.705(9)$ \\
\hline$C(4)-C(5)$ & $1.504(10)$ & $\mathrm{B}(1)-\mathrm{B}\left(8^{\prime \prime}\right)$ & $1.762(12)$ \\
\hline
\end{tabular}




\begin{tabular}{|c|c|c|c|}
\hline$C(5)-C(8)$ & $1.501(9)$ & $\mathrm{B}(1)-\mathrm{B}(3)$ & $1.777(10)$ \\
\hline$C(5)-C(7)$ & $1.546(8)$ & $\mathrm{B}(1)-\mathrm{B}(2)$ & $1.782(14)$ \\
\hline$C(5)-C(6)$ & $1.545(8)$ & $\mathrm{B}(1)-\mathrm{B}\left(18^{\prime \prime}\right)$ & $1.785(14)$ \\
\hline$C(8)-C(9)$ & $1.446(10)$ & $\mathrm{B}(1)-\mathrm{B}\left(7^{\prime \prime}\right)$ & $1.795(12)$ \\
\hline $\mathrm{B}(2)-\mathrm{B}(3)$ & $1.750(13)$ & $\mathrm{B}(10)-\mathrm{H}(62)$ & $1.06(6)$ \\
\hline $\mathrm{B}(2)-\mathrm{B}(4)$ & $1.769(11)$ & $\mathrm{B}(10)-\mathrm{B}\left(11^{\prime \prime \prime}\right)$ & $1.783(11)$ \\
\hline $\mathrm{B}(2)-\mathrm{B}\left(5^{\prime \prime}\right)$ & $1.792(10)$ & $\mathrm{B}(10)-\mathrm{B}(15)$ & $1.791(11)$ \\
\hline $\mathrm{B}(2)-\mathrm{B}\left(7^{\prime \prime}\right)$ & $1.793(12)$ & $\mathrm{B}(10)-\mathrm{B}\left(13^{\prime \prime}\right)$ & $1.798(11)$ \\
\hline $\mathrm{B}(3)-\mathrm{B}\left(18^{\prime \prime}\right)$ & $1.767(13)$ & $\mathrm{B}(10)-\mathrm{B}(12)$ & $1.811(10)$ \\
\hline $\mathrm{B}(3)-\mathrm{B}(4)$ & $1.796(11)$ & $\mathrm{B}(11)-\mathrm{B}\left(17^{\prime \prime \prime}\right)$ & $1.771(11)$ \\
\hline $\mathrm{B}(4)-\mathrm{B}\left(5^{\prime \prime}\right)$ & $1.800(11)$ & $\mathrm{B}(11)-\mathrm{B}\left(9^{\prime \prime \prime}\right)$ & $1.779(12)$ \\
\hline $\mathrm{B}(5)-\mathrm{B}\left(2^{\prime \prime}\right)$ & $1.792(10)$ & $\mathrm{B}(11)-\mathrm{B}\left(10^{\prime \prime \prime}\right)$ & $1.783(11)$ \\
\hline $\mathrm{B}(5)-\mathrm{B}(6)$ & $1.798(12)$ & $\mathrm{B}(11)-\mathrm{B}(13)$ & $1.791(11)$ \\
\hline $\mathrm{B}(5)-\mathrm{B}\left(4^{\prime \prime}\right)$ & $1.800(11)$ & $\mathrm{B}(12)-\mathrm{B}(15)$ & $1.787(10)$ \\
\hline $\mathrm{B}(5)-\mathrm{B}(7)$ & $1.808(11)$ & $\mathrm{B}(12)-\mathrm{B}(14)$ & $1.809(11)$ \\
\hline $\mathrm{B}(6)-\mathrm{H}(56)$ & $1.15(5)$ & $\mathrm{B}(12)-\mathrm{B}\left(13^{\prime \prime \prime}\right)$ & $1.819(12)$ \\
\hline $\mathrm{B}(6)-\mathrm{B}(7)$ & $1.773(11)$ & $\mathrm{B}(13)-\mathrm{B}\left(10^{\prime \prime \prime}\right)$ & $1.798(11)$ \\
\hline $\mathrm{B}(6)-\mathrm{B}(8)$ & $1.800(11)$ & $\mathrm{B}(13)-\mathrm{B}\left(12^{\prime \prime \prime}\right)$ & $1.819(12)$ \\
\hline $\mathrm{B}(7)-\mathrm{B}(8)$ & $1.764(12)$ & $\mathrm{B}(14)-\mathrm{B}(15)$ & $1.785(10)$ \\
\hline $\mathrm{B}(7)-\mathrm{B}\left(2^{\prime \prime}\right)$ & $1.793(12)$ & $\mathrm{B}(14)-\mathrm{B}(16)$ & $1.799(11)$ \\
\hline $\mathrm{B}(7)-\mathrm{B}\left(1^{\prime \prime}\right)$ & $1.795(12)$ & $\mathrm{B}(15)-\mathrm{B}(16)$ & $1.770(13)$ \\
\hline $\mathrm{B}(8)-\mathrm{B}\left(1^{\prime \prime}\right)$ & $1.762(12)$ & $\mathrm{B}(16)-\mathrm{B}(17)$ & $1.745(13)$ \\
\hline $\mathrm{B}(8)-\mathrm{B}(18)$ & $1.776(12)$ & $\mathrm{B}(17)-\mathrm{C}\left(18^{\prime \prime \prime}\right)$ & $1.739(10)$ \\
\hline $\mathrm{B}(9)-\mathrm{B}(15)$ & $1.758(13)$ & $\mathrm{B}(17)-\mathrm{B}\left(11^{\prime \prime \prime}\right)$ & $1.771(11)$ \\
\hline $\mathrm{B}(9)-\mathrm{B}(17)$ & $1.766(13)$ & $\mathrm{B}(18)-\mathrm{C}\left(20^{\prime \prime}\right)$ & $1.740(10)$ \\
\hline $\mathrm{B}(9)-\mathrm{B}(16)$ & $1.774(11)$ & $\mathrm{B}(18)-\mathrm{B}\left(3^{\prime \prime}\right)$ & $1.767(13)$ \\
\hline $\mathrm{B}(9)-\mathrm{B}\left(11^{\prime \prime \prime}\right)$ & $1.779(12)$ & $\mathrm{B}(18)-\mathrm{B}\left(1^{\prime \prime}\right)$ & $1.785(14)$ \\
\hline $\mathrm{B}(9)-\mathrm{B}(10)$ & $1.781(11)$ & & \\
\hline
\end{tabular}

Primed labels refer to atoms generated by the following symmetry transformations: single prime: $\mathrm{x},-\mathrm{y}+3 / 2, \mathrm{z}$; double prime: $-\mathrm{x}+1,-\mathrm{y}+2, \mathrm{z}$; triple prime: $-\mathrm{x},-\mathrm{y}+2, \mathrm{z}$ 
Table S23. Bond angles (in deg) for $\left[\mathrm{L}^{\Delta \Lambda} \mathrm{Fe}^{\mathrm{II}}\left(\mathrm{NCCH}_{3}\right)\right]\left[\left(\mathrm{C}_{2} \mathrm{~B}_{9} \mathrm{H}_{11}\right)_{2} \mathrm{Co}\right]_{2} \cdot 2 \mathrm{CH}_{3} \mathrm{CN} \cdot 2 o-\mathrm{C}_{6} \mathrm{H}_{4} \mathrm{Cl}_{2}$

\begin{tabular}{|c|c|c|c|}
\hline Bond angle & Angle & Bond angle & Angle \\
\hline $\mathrm{N}(3)-\mathrm{Fe}(1)-\mathrm{N}(2)$ & $110.0(2)$ & $\mathrm{C}\left(20^{\prime \prime}\right)-\mathrm{Co}(1)-\mathrm{C}(20)$ & $134.9(4)$ \\
\hline $\mathrm{N}(3)-\mathrm{Fe}(1)-\mathrm{N}\left(2^{\prime}\right)$ & $110.0(2)$ & $\mathrm{C}\left(20^{\prime \prime}\right)-\mathrm{Co}(1)-\mathrm{C}\left(21^{\prime \prime}\right)$ & $100.8(3)$ \\
\hline $\mathrm{N}(2)-\mathrm{Fe}(1)-\mathrm{N}\left(2^{\prime}\right)$ & $78.6(3)$ & $\mathrm{C}(20)-\mathrm{Co}(1)-\mathrm{C}\left(21^{\prime \prime}\right)$ & $47.1(3)$ \\
\hline $\mathrm{N}(3)-\mathrm{Fe}(1)-\mathrm{N}(1)$ & $110.1(2)$ & $\mathrm{C}\left(20^{\prime \prime}\right)-\mathrm{Co}(1)-\mathrm{C}(21)$ & $47.1(3)$ \\
\hline $\mathrm{N}(2)-\mathrm{Fe}(1)-\mathrm{N}(1)$ & $87.44(18)$ & $\mathrm{C}(20)-\mathrm{Co}(1)-\mathrm{C}(21)$ & $100.8(3)$ \\
\hline $\mathrm{N}\left(2^{\prime}\right)-\mathrm{Fe}(1)-\mathrm{N}(1)$ & 139.92(19) & $\mathrm{C}\left(21^{\prime \prime}\right)-\mathrm{Co}(1)-\mathrm{C}(21)$ & $100.0(4)$ \\
\hline $\mathrm{N}(3)-\mathrm{Fe}(1)-\mathrm{N}\left(1^{\prime}\right)$ & $110.1(2)$ & $\mathrm{C}\left(20^{\prime \prime}\right)-\mathrm{Co}(1)-\mathrm{B}\left(4^{\prime \prime}\right)$ & $49.2(3)$ \\
\hline $\mathrm{N}(2)-\mathrm{Fe}(1)-\mathrm{N}\left(1^{\prime}\right)$ & 139.92(19) & $\mathrm{C}(20)-\mathrm{Co}(1)-\mathrm{B}\left(4^{\prime \prime}\right)$ & $174.9(3)$ \\
\hline $\mathrm{N}\left(2^{\prime}\right)-\mathrm{Fe}(1)-\mathrm{N}\left(1^{\prime}\right)$ & $87.44(18)$ & $\mathrm{C}\left(21^{\prime \prime}\right)-\mathrm{Co}(1)-\mathrm{B}\left(4^{\prime \prime}\right)$ & $132.4(3)$ \\
\hline $\mathrm{N}(1)-\mathrm{Fe}(1)-\mathrm{N}\left(1^{\prime}\right)$ & $79.5(3)$ & $\mathrm{C}(21)-\mathrm{Co}(1)-\mathrm{B}\left(4^{\prime \prime}\right)$ & $84.3(3)$ \\
\hline $\mathrm{C}\left(20^{\prime \prime}\right)-\mathrm{Co}(1)-\mathrm{B}(4)$ & $174.9(3)$ & $\mathrm{C}(19)-\mathrm{Co}(2)-\mathrm{B}(12)$ & $83.9(3)$ \\
\hline $\mathrm{C}(20)-\mathrm{Co}(1)-\mathrm{B}(4)$ & $49.2(3)$ & $\mathrm{C}\left(18^{\prime \prime \prime}\right)-\mathrm{Co}(2)-\mathrm{B}(12)$ & $83.6(3)$ \\
\hline $\mathrm{C}\left(21^{\prime \prime}\right)-\mathrm{Co}(1)-\mathrm{B}(4)$ & $84.3(3)$ & $\mathrm{C}(18)-\mathrm{Co}(2)-\mathrm{B}(12)$ & $173.4(3)$ \\
\hline $\mathrm{C}(21)-\mathrm{Co}(1)-\mathrm{B}(4)$ & $132.4(3)$ & $\mathrm{B}\left(12^{\prime \prime \prime}\right)-\mathrm{Co}(2)-\mathrm{B}(12)$ & $91.6(4)$ \\
\hline $\mathrm{B}\left(4^{\prime \prime}\right)-\mathrm{Co}(1)-\mathrm{B}(4)$ & $126.8(5)$ & $\mathrm{C}\left(19^{\prime \prime \prime}\right)-\mathrm{Co}(2)-\mathrm{B}\left(13^{\prime \prime \prime}\right)$ & $96.1(3)$ \\
\hline $\mathrm{C}\left(20^{\prime \prime}\right)-\mathrm{Co}(1)-\mathrm{B}\left(6^{\prime \prime}\right)$ & $95.7(3)$ & $\mathrm{C}(19)-\mathrm{Co}(2)-\mathrm{B}\left(13^{\prime \prime \prime}\right)$ & $82.6(3)$ \\
\hline $\mathrm{C}(20)-\mathrm{Co}(1)-\mathrm{B}\left(6^{\prime \prime}\right)$ & $83.6(3)$ & $\mathrm{C}\left(18^{\prime \prime \prime}\right)-\mathrm{Co}(2)-\mathrm{B}\left(13^{\prime \prime \prime}\right)$ & $47.4(3)$ \\
\hline $\mathrm{C}\left(21^{\prime \prime}\right)-\mathrm{Co}(1)-\mathrm{B}\left(6^{\prime \prime}\right)$ & $48.4(3)$ & $\mathrm{C}(18)-\mathrm{Co}(2)-\mathrm{B}\left(13^{\prime \prime \prime}\right)$ & $129.6(3)$ \\
\hline $\mathrm{C}(21)-\mathrm{Co}(1)-\mathrm{B}\left(6^{\prime \prime}\right)$ & $130.1(4)$ & $\mathrm{B}\left(12^{\prime \prime \prime}\right)-\mathrm{Co}(2)-\mathrm{B}\left(13^{\prime \prime \prime}\right)$ & $131.7(4)$ \\
\hline $\mathrm{B}\left(4^{\prime \prime}\right)-\mathrm{Co}(1)-\mathrm{B}\left(6^{\prime \prime}\right)$ & $93.2(3)$ & $\mathrm{B}(12)-\mathrm{Co}(2)-\mathrm{B}\left(13^{\prime \prime \prime}\right)$ & $51.5(3)$ \\
\hline $\mathrm{B}(4)-\mathrm{Co}(1)-\mathrm{B}\left(6^{\prime \prime}\right)$ & $87.7(3)$ & $\mathrm{C}\left(19^{\prime \prime \prime}\right)-\mathrm{Co}(2)-\mathrm{B}(13)$ & $82.6(3)$ \\
\hline $\mathrm{C}\left(20^{\prime \prime}\right)-\mathrm{Co}(1)-\mathrm{B}(6)$ & $83.6(3)$ & $\mathrm{C}(19)-\mathrm{Co}(2)-\mathrm{B}(13)$ & $96.1(3)$ \\
\hline $\mathrm{C}(20)-\mathrm{Co}(1)-\mathrm{B}(6)$ & $95.7(3)$ & $\mathrm{C}\left(18^{\prime \prime \prime}\right)-\mathrm{Co}(2)-\mathrm{B}(13)$ & $129.6(3)$ \\
\hline $\mathrm{C}\left(21^{\prime \prime}\right)-\mathrm{Co}(1)-\mathrm{B}(6)$ & $130.1(3)$ & $\mathrm{C}(18)-\mathrm{Co}(2)-\mathrm{B}(13)$ & $47.4(3)$ \\
\hline $\mathrm{C}(21)-\mathrm{Co}(1)-\mathrm{B}(6)$ & $48.4(3)$ & $\mathrm{B}\left(12^{\prime \prime \prime}\right)-\mathrm{Co}(2)-\mathrm{B}(13)$ & $51.5(3)$ \\
\hline $\mathrm{B}\left(4^{\prime \prime}\right)-\mathrm{Co}(1)-\mathrm{B}(6)$ & $87.7(3)$ & $\mathrm{B}(12)-\mathrm{Co}(2)-\mathrm{B}(13)$ & $131.7(4)$ \\
\hline $\mathrm{B}(4)-\mathrm{Co}(1)-\mathrm{B}(6)$ & $93.2(3)$ & $\mathrm{B}\left(13^{\prime \prime \prime}\right)-\mathrm{Co}(2)-\mathrm{B}(13)$ & $176.5(6)$ \\
\hline $\mathrm{B}\left(6^{\prime \prime}\right)-\mathrm{Co}(1)-\mathrm{B}(6)$ & $178.2(6)$ & $\mathrm{C}\left(19^{\prime \prime \prime}\right)-\mathrm{Co}(2)-\mathrm{B}(14)$ & $173.5(3)$ \\
\hline $\mathrm{C}\left(20^{\prime \prime}\right)-\mathrm{Co}(1)-\mathrm{B}(5)$ & $84.5(3)$ & $\mathrm{C}(19)-\mathrm{Co}(2)-\mathrm{B}(14)$ & $49.2(3)$ \\
\hline $\mathrm{C}(20)-\mathrm{Co}(1)-\mathrm{B}(5)$ & $128.9(3)$ & $\mathrm{C}\left(18^{\prime \prime \prime}\right)-\mathrm{Co}(2)-\mathrm{B}(14)$ & $83.9(3)$ \\
\hline $\mathrm{C}\left(21^{\prime \prime}\right)-\mathrm{Co}(1)-\mathrm{B}(5)$ & $174.6(3)$ & $\mathrm{C}(18)-\mathrm{Co}(2)-\mathrm{B}(14)$ & $133.0(3)$ \\
\hline $\mathrm{C}(21)-\mathrm{Co}(1)-\mathrm{B}(5)$ & $84.2(3)$ & $\mathrm{B}\left(12^{\prime \prime \prime}\right)-\mathrm{Co}(2)-\mathrm{B}(14)$ & $89.6(3)$ \\
\hline $\mathrm{B}\left(4^{\prime \prime}\right)-\mathrm{Co}(1)-\mathrm{B}(5)$ & $51.1(3)$ & $\mathrm{B}(12)-\mathrm{Co}(2)-\mathrm{B}(14)$ & $51.2(3)$ \\
\hline $\mathrm{B}(4)-\mathrm{Co}(1)-\mathrm{B}(5)$ & $90.4(3)$ & $\mathrm{B}\left(13^{\prime \prime \prime}\right)-\mathrm{Co}(2)-\mathrm{B}(14)$ & $88.2(3)$ \\
\hline $\mathrm{B}\left(6^{\prime \prime}\right)-\mathrm{Co}(1)-\mathrm{B}(5)$ & $130.9(4)$ & $\mathrm{B}(13)-\mathrm{Co}(2)-\mathrm{B}(14)$ & $93.4(3)$ \\
\hline $\mathrm{B}(6)-\mathrm{Co}(1)-\mathrm{B}(5)$ & $50.8(3)$ & $\mathrm{C}\left(19^{\prime \prime \prime}\right)-\mathrm{Co}(2)-\mathrm{B}\left(14^{\prime \prime \prime}\right)$ & $49.2(3)$ \\
\hline $\mathrm{C}\left(20^{\prime \prime}\right)-\mathrm{Co}(1)-\mathrm{B}\left(5^{\prime \prime}\right)$ & $128.9(3)$ & $\mathrm{C}(19)-\mathrm{Co}(2)-\mathrm{B}\left(14^{\prime \prime \prime}\right)$ & $173.5(3)$ \\
\hline $\mathrm{C}(20)-\mathrm{Co}(1)-\mathrm{B}\left(5^{\prime \prime}\right)$ & $84.5(3)$ & $\mathrm{C}\left(18^{\prime \prime \prime}\right)-\mathrm{Co}(2)-\mathrm{B}\left(14^{\prime \prime \prime}\right)$ & $133.0(3)$ \\
\hline $\mathrm{C}\left(21^{\prime \prime}\right)-\mathrm{Co}(1)-\mathrm{B}\left(5^{\prime \prime}\right)$ & $84.2(3)$ & $\mathrm{C}(18)-\mathrm{Co}(2)-\mathrm{B}\left(14^{\prime \prime \prime}\right)$ & $83.9(3)$ \\
\hline
\end{tabular}




\begin{tabular}{|c|c|}
\hline $\mathrm{C}(21)-\mathrm{Co}(1)-\mathrm{B}\left(5^{\prime \prime}\right)$ & $174.6(3)$ \\
\hline $\mathrm{B}\left(4^{\prime \prime}\right)-\mathrm{Co}(1)-\mathrm{B}\left(5^{\prime \prime}\right)$ & $90.4(3)$ \\
\hline $\mathrm{B}(4)-\mathrm{Co}(1)-\mathrm{B}\left(5^{\prime \prime}\right)$ & $51.1(3)$ \\
\hline $\mathrm{B}\left(6^{\prime \prime}\right)-\mathrm{Co}(1)-\mathrm{B}\left(5^{\prime \prime}\right)$ & $50.8(3)$ \\
\hline $\mathrm{B}(6)-\mathrm{Co}(1)-\mathrm{B}\left(5^{\prime \prime}\right)$ & $130.9(4)$ \\
\hline $\mathrm{B}(5)-\mathrm{Co}(1)-\mathrm{B}\left(5^{\prime \prime}\right)$ & $91.9(5)$ \\
\hline $\mathrm{C}\left(19^{\prime \prime \prime}\right)-\mathrm{Co}(2)-\mathrm{C}(19)$ & $136.1(5)$ \\
\hline $\mathrm{C}\left(19^{\prime \prime \prime}\right)-\mathrm{Co}(2)-\mathrm{C}\left(18^{\prime \prime \prime}\right)$ & $102.5(3)$ \\
\hline $\mathrm{C}(19)-\mathrm{Co}(2)-\mathrm{C}\left(18^{\prime \prime \prime}\right)$ & $46.4(3)$ \\
\hline $\mathrm{C}\left(19^{\prime \prime \prime}\right)-\mathrm{Co}(2)-\mathrm{C}(18)$ & $46.4(3)$ \\
\hline $\mathrm{C}(19)-\mathrm{Co}(2)-\mathrm{C}(18)$ & $102.5(3)$ \\
\hline $\mathrm{C}\left(18^{\prime \prime \prime}\right)-\mathrm{Co}(2)-\mathrm{C}(18)$ & $101.5(4)$ \\
\hline $\mathrm{C}\left(19^{\prime \prime \prime}\right)-\mathrm{Co}(2)-\mathrm{B}\left(12^{\prime \prime \prime}\right)$ & $83.9(3)$ \\
\hline $\mathrm{C}(19)-\mathrm{Co}(2)-\mathrm{B}\left(12^{\prime \prime \prime}\right)$ & $128.8(3)$ \\
\hline $\mathrm{C}\left(18^{\prime \prime \prime}\right)-\mathrm{Co}(2)-\mathrm{B}\left(12^{\prime \prime \prime}\right)$ & $173.4(3)$ \\
\hline $\mathrm{C}(18)-\mathrm{Co}(2)-\mathrm{B}\left(12^{\prime \prime \prime}\right)$ & $83.6(3)$ \\
\hline $\mathrm{C}\left(19^{\prime \prime \prime}\right)-\mathrm{Co}(2)-\mathrm{B}(12)$ & $128.8(3)$ \\
\hline $\mathrm{C}(15)-\mathrm{C}(1)-\mathrm{C}\left(1^{\prime}\right)$ & $58.2(3)$ \\
\hline$C(3)-C(2)-C(1)$ & $106.7(6)$ \\
\hline$C(2)-C(3)-C(4)$ & $107.9(6)$ \\
\hline $\mathrm{N}(1)-\mathrm{C}(4)-\mathrm{C}(3)$ & $111.5(6)$ \\
\hline $\mathrm{N}(1)-\mathrm{C}(4)-\mathrm{C}(5)$ & $122.4(6)$ \\
\hline$C(3)-C(4)-C(5)$ & $126.1(6)$ \\
\hline$C(8)-C(5)-C(4)$ & $110.3(4)$ \\
\hline$C(8)-C(5)-C(7)$ & $110.1(6)$ \\
\hline$C(4)-C(5)-C(7)$ & $111.1(6)$ \\
\hline$C(8)-C(5)-C(6)$ & $109.0(6)$ \\
\hline$C(4)-C(5)-C(6)$ & $108.0(6)$ \\
\hline$C(7)-C(5)-C(6)$ & $108.3(5)$ \\
\hline $\mathrm{N}(2)-\mathrm{C}(8)-\mathrm{C}(9)$ & $110.2(6)$ \\
\hline $\mathrm{N}(2)-\mathrm{C}(8)-\mathrm{C}(5)$ & $120.7(5)$ \\
\hline $\mathrm{C}(9)-\mathrm{C}(8)-\mathrm{C}(5)$ & $129.2(5)$ \\
\hline $\mathrm{C}(10)-\mathrm{C}(9)-\mathrm{C}(8)$ & $109.5(6)$ \\
\hline$C(9)-C(10)-C(11)$ & $106.2(6)$ \\
\hline $\mathrm{N}(2)-\mathrm{C}(11)-\mathrm{C}(10)$ & $107.4(5)$ \\
\hline $\mathrm{N}(2)-\mathrm{C}(11)-\mathrm{C}(12)$ & $120.6(6)$ \\
\hline $\mathrm{C}(10)-\mathrm{C}(11)-\mathrm{C}(12)$ & $124.6(7)$ \\
\hline $\mathrm{N}(2)-\mathrm{C}(11)-\mathrm{C}\left(11^{\prime}\right)$ & $112.9(3)$ \\
\hline $\mathrm{C}(10)-\mathrm{C}(11)-\mathrm{C}\left(11^{\prime}\right)$ & $125.5(4)$ \\
\hline$C(12)-C(11)-C\left(11^{\prime}\right)$ & $59.0(3)$ \\
\hline $\mathrm{C}(11)-\mathrm{C}(12)-\mathrm{C}\left(11^{\prime}\right)$ & $62.1(6)$ \\
\hline$C(11)-C(12)-C(14)$ & $117.4(8)$ \\
\hline $\mathrm{C}\left(11^{\prime}\right)-\mathrm{C}(12)-\mathrm{C}(14)$ & $117.4(8)$ \\
\hline
\end{tabular}

\begin{tabular}{|c|c|}
\hline $\mathrm{B}\left(12^{\prime \prime \prime}\right)-\mathrm{Co}(2)-\mathrm{B}\left(14^{\prime \prime \prime}\right)$ & $51.2(3)$ \\
\hline $\mathrm{B}(12)-\mathrm{Co}(2)-\mathrm{B}\left(14^{\prime \prime \prime}\right)$ & $89.6(3)$ \\
\hline $\mathrm{B}\left(13^{\prime \prime \prime}\right)-\mathrm{Co}(2)-\mathrm{B}\left(14^{\prime \prime \prime}\right)$ & $93.4(3)$ \\
\hline $\mathrm{B}(13)-\mathrm{Co}(2)-\mathrm{B}\left(14^{\prime \prime \prime}\right)$ & $88.2(3)$ \\
\hline $\mathrm{B}(14)-\mathrm{Co}(2)-\mathrm{B}\left(14^{\prime \prime \prime}\right)$ & $125.9(5)$ \\
\hline $\mathrm{C}(4)-\mathrm{N}(1)-\mathrm{C}(1)$ & $106.0(5)$ \\
\hline $\mathrm{C}(4)-\mathrm{N}(1)-\mathrm{Fe}(1)$ & $125.9(4)$ \\
\hline $\mathrm{C}(1)-\mathrm{N}(1)-\mathrm{Fe}(1)$ & $117.7(3)$ \\
\hline $\mathrm{C}(8)-\mathrm{N}(2)-\mathrm{C}(11)$ & $106.8(5)$ \\
\hline $\mathrm{C}(8)-\mathrm{N}(2)-\mathrm{Fe}(1)$ & $125.9(4)$ \\
\hline $\mathrm{C}(11)-\mathrm{N}(2)-\mathrm{Fe}(1)$ & $117.6(4)$ \\
\hline $\mathrm{C}(22)-\mathrm{N}(3)-\mathrm{Fe}(1)$ & $179.9(8)$ \\
\hline $\mathrm{N}(1)-\mathrm{C}(1)-\mathrm{C}(2)$ & $107.8(5)$ \\
\hline $\mathrm{N}(1)-\mathrm{C}(1)-\mathrm{C}(15)$ & 119.1(6) \\
\hline$C(2)-C(1)-C(15)$ & $126.9(7)$ \\
\hline $\mathrm{N}(1)-\mathrm{C}(1)-\mathrm{C}\left(1^{\prime}\right)$ & $112.3(3)$ \\
\hline $\mathrm{C}(2)-\mathrm{C}(1)-\mathrm{C}\left(1^{\prime}\right)$ & $124.4(4)$ \\
\hline $\mathrm{C}\left(18^{\prime \prime \prime}\right)-\mathrm{C}(19)-\mathrm{B}(14)$ & $112.9(6)$ \\
\hline $\mathrm{B}(16)-\mathrm{C}(19)-\mathrm{B}(14)$ & $63.5(5)$ \\
\hline $\mathrm{C}\left(18^{\prime \prime \prime}\right)-\mathrm{C}(19)-\mathrm{B}(17)$ & $62.7(4)$ \\
\hline $\mathrm{B}(16)-\mathrm{C}(19)-\mathrm{B}(17)$ & $61.2(5)$ \\
\hline $\mathrm{B}(14)-\mathrm{C}(19)-\mathrm{B}(17)$ & $115.2(6)$ \\
\hline $\mathrm{C}\left(18^{\prime \prime \prime}\right)-\mathrm{C}(19)-\mathrm{Co}(2)$ & $67.4(3)$ \\
\hline $\mathrm{B}(16)-\mathrm{C}(19)-\mathrm{Co}(2)$ & $124.9(6)$ \\
\hline $\mathrm{B}(14)-\mathrm{C}(19)-\mathrm{Co}(2)$ & $67.3(4)$ \\
\hline $\mathrm{B}(17)-\mathrm{C}(19)-\mathrm{Co}(2)$ & $126.0(4)$ \\
\hline $\mathrm{C}\left(21^{\prime \prime}\right)-\mathrm{C}(20)-\mathrm{B}(3)$ & $110.8(5)$ \\
\hline $\mathrm{C}\left(21^{\prime \prime}\right)-\mathrm{C}(20)-\mathrm{B}(4)$ & $111.9(6)$ \\
\hline $\mathrm{B}(3)-\mathrm{C}(20)-\mathrm{B}(4)$ & $63.6(5)$ \\
\hline $\mathrm{C}\left(21^{\prime \prime}\right)-\mathrm{C}(20)-\mathrm{B}\left(18^{\prime \prime}\right)$ & $62.3(4)$ \\
\hline $\mathrm{B}(3)-\mathrm{C}(20)-\mathrm{B}\left(18^{\prime \prime}\right)$ & $61.9(5)$ \\
\hline $\mathrm{B}(4)-\mathrm{C}(20)-\mathrm{B}\left(18^{\prime \prime}\right)$ & $116.0(6)$ \\
\hline $\mathrm{C}\left(21^{\prime \prime}\right)-\mathrm{C}(20)-\mathrm{Co}(1)$ & $67.3(3)$ \\
\hline $\mathrm{B}(3)-\mathrm{C}(20)-\mathrm{Co}(1)$ & $124.4(5)$ \\
\hline $\mathrm{B}(4)-\mathrm{C}(20)-\mathrm{Co}(1)$ & $66.7(4)$ \\
\hline $\mathrm{B}\left(18^{\prime \prime}\right)-\mathrm{C}(20)-\mathrm{Co}(1)$ & $125.8(5)$ \\
\hline $\mathrm{C}\left(20^{\prime \prime}\right)-\mathrm{C}(21)-\mathrm{B}(8)$ & $110.7(5)$ \\
\hline $\mathrm{C}\left(20^{\prime \prime}\right)-\mathrm{C}(21)-\mathrm{B}(6)$ & $111.1(6)$ \\
\hline $\mathrm{B}(8)-\mathrm{C}(21)-\mathrm{B}(6)$ & $64.2(5)$ \\
\hline $\mathrm{C}\left(20^{\prime \prime}\right)-\mathrm{C}(21)-\mathrm{B}(18)$ & $61.8(4)$ \\
\hline $\mathrm{B}(8)-\mathrm{C}(21)-\mathrm{B}(18)$ & $62.2(5)$ \\
\hline $\mathrm{B}(6)-\mathrm{C}(21)-\mathrm{B}(18)$ & $116.1(6)$ \\
\hline $\mathrm{C}\left(20^{\prime \prime}\right)-\mathrm{C}(21)-\mathrm{Co}(1)$ & $65.6(3)$ \\
\hline
\end{tabular}




\begin{tabular}{|c|c|}
\hline$C(11)-C(12)-C(13)$ & $119.0(7)$ \\
\hline $\mathrm{C}\left(11^{\prime}\right)-\mathrm{C}(12)-\mathrm{C}(13)$ & $119.0(7)$ \\
\hline$C(14)-C(12)-C(13)$ & $113.1(10)$ \\
\hline$C(17)-C(15)-C(16)$ & $111.7(9)$ \\
\hline$C(17)-C(15)-C\left(1^{\prime}\right)$ & $117.4(7)$ \\
\hline$C(16)-C(15)-C\left(1^{\prime}\right)$ & $119.6(7)$ \\
\hline$C(17)-C(15)-C(1)$ & $117.4(7)$ \\
\hline$C(16)-C(15)-C(1)$ & $119.6(7)$ \\
\hline$C\left(1^{\prime}\right)-C(15)-C(1)$ & $63.6(6)$ \\
\hline $\mathrm{C}\left(19^{\prime \prime \prime}\right)-\mathrm{C}(18)-\mathrm{B}(13)$ & $112.4(6)$ \\
\hline $\mathrm{C}\left(19^{\prime \prime \prime}\right)-\mathrm{C}(18)-\mathrm{B}(11)$ & $111.7(5)$ \\
\hline $\mathrm{B}(13)-\mathrm{C}(18)-\mathrm{B}(11)$ & $64.1(5)$ \\
\hline $\mathrm{C}\left(19^{\prime \prime \prime}\right)-\mathrm{C}(18)-\mathrm{B}\left(17^{\prime \prime \prime}\right)$ & $62.1(4)$ \\
\hline $\mathrm{B}(13)-\mathrm{C}(18)-\mathrm{B}\left(17^{\prime \prime \prime}\right)$ & $115.5(6)$ \\
\hline $\mathrm{B}(11)-\mathrm{C}(18)-\mathrm{B}\left(17^{\prime \prime \prime}\right)$ & $61.8(4)$ \\
\hline $\mathrm{C}\left(19^{\prime \prime \prime}\right)-\mathrm{C}(18)-\mathrm{Co}(2)$ & $66.2(3)$ \\
\hline $\mathrm{B}(13)-\mathrm{C}(18)-\mathrm{Co}(2)$ & $67.5(4)$ \\
\hline $\mathrm{B}(11)-\mathrm{C}(18)-\mathrm{Co}(2)$ & $125.5(5)$ \\
\hline $\mathrm{B}\left(17^{\prime \prime \prime}\right)-\mathrm{C}(18)-\mathrm{Co}(2)$ & $124.3(5)$ \\
\hline $\mathrm{C}\left(18^{\prime \prime \prime}\right)-\mathrm{C}(19)-\mathrm{B}(16)$ & $111.3(5)$ \\
\hline $\mathrm{B}(3)-\mathrm{B}(1)-\mathrm{B}\left(18^{\prime \prime}\right)$ & $59.5(5)$ \\
\hline $\mathrm{B}(2)-\mathrm{B}(1)-\mathrm{B}\left(18^{\prime \prime}\right)$ & $107.2(5)$ \\
\hline $\mathrm{B}\left(8^{\prime \prime}\right)-\mathrm{B}(1)-\mathrm{B}\left(7^{\prime \prime}\right)$ & $59.4(4)$ \\
\hline $\mathrm{B}(3)-\mathrm{B}(1)-\mathrm{B}\left(7^{\prime \prime}\right)$ & $107.0(6)$ \\
\hline $\mathrm{B}(2)-\mathrm{B}(1)-\mathrm{B}\left(7^{\prime \prime}\right)$ & $60.1(5)$ \\
\hline $\mathrm{B}\left(18^{\prime \prime}\right)-\mathrm{B}(1)-\mathrm{B}\left(7^{\prime \prime}\right)$ & $108.2(6)$ \\
\hline $\mathrm{B}(3)-\mathrm{B}(2)-\mathrm{B}(4)$ & $61.4(5)$ \\
\hline $\mathrm{B}(3)-\mathrm{B}(2)-\mathrm{B}(1)$ & $60.4(5)$ \\
\hline $\mathrm{B}(4)-\mathrm{B}(2)-\mathrm{B}(1)$ & $110.4(7)$ \\
\hline $\mathrm{B}(3)-\mathrm{B}(2)-\mathrm{B}\left(5^{\prime \prime}\right)$ & $110.0(6)$ \\
\hline $\mathrm{B}(4)-\mathrm{B}(2)-\mathrm{B}\left(5^{\prime \prime}\right)$ & $60.7(4)$ \\
\hline $\mathrm{B}(1)-\mathrm{B}(2)-\mathrm{B}\left(5^{\prime \prime}\right)$ & $110.3(6)$ \\
\hline $\mathrm{B}(3)-\mathrm{B}(2)-\mathrm{B}\left(7^{\prime \prime}\right)$ & $108.3(6)$ \\
\hline $\mathrm{B}(4)-\mathrm{B}(2)-\mathrm{B}\left(7^{\prime \prime}\right)$ & $109.0(6)$ \\
\hline $\mathrm{B}(1)-\mathrm{B}(2)-\mathrm{B}\left(7^{\prime \prime}\right)$ & $60.3(5)$ \\
\hline $\mathrm{B}\left(5^{\prime \prime}\right)-\mathrm{B}(2)-\mathrm{B}\left(7^{\prime \prime}\right)$ & $60.6(4)$ \\
\hline $\mathrm{C}(20)-\mathrm{B}(3)-\mathrm{B}(2)$ & $104.2(5)$ \\
\hline $\mathrm{C}(20)-\mathrm{B}(3)-\mathrm{B}\left(18^{\prime \prime}\right)$ & $60.3(4)$ \\
\hline $\mathrm{B}(2)-\mathrm{B}(3)-\mathrm{B}\left(18^{\prime \prime}\right)$ & $109.4(5)$ \\
\hline $\mathrm{C}(20)-\mathrm{B}(3)-\mathrm{B}(1)$ & $105.7(5)$ \\
\hline $\mathrm{B}(2)-\mathrm{B}(3)-\mathrm{B}(1)$ & $60.7(5)$ \\
\hline $\mathrm{B}\left(18^{\prime \prime}\right)-\mathrm{B}(3)-\mathrm{B}(1)$ & $60.5(6)$ \\
\hline $\mathrm{C}(20)-\mathrm{B}(3)-\mathrm{B}(4)$ & $58.7(4)$ \\
\hline
\end{tabular}

\begin{tabular}{|c|c|}
\hline $\mathrm{B}(8)-\mathrm{C}(21)-\mathrm{Co}(1)$ & $124.7(5)$ \\
\hline $\mathrm{B}(6)-\mathrm{C}(21)-\mathrm{Co}(1)$ & $66.8(3)$ \\
\hline $\mathrm{B}(18)-\mathrm{C}(21)-\mathrm{Co}(1)$ & $123.9(5)$ \\
\hline$N(3)-C(22)-C(23)$ & $175.4(11)$ \\
\hline$N(4)-C(24)-C(25)$ & $176.0(15)$ \\
\hline$N(5)-C(26)-C(27)$ & $175(3)$ \\
\hline $\mathrm{C}(29)-\mathrm{C}(28)-\mathrm{C}(33)$ & $120.3(9)$ \\
\hline $\mathrm{C}(29)-\mathrm{C}(28)-\mathrm{Cl}(1)$ & $119.3(6)$ \\
\hline $\mathrm{C}(33)-\mathrm{C}(28)-\mathrm{Cl}(1)$ & $120.4(7)$ \\
\hline$C(28)-C(29)-C(30)$ & $119.7(7)$ \\
\hline$C(31)-C(30)-C(29)$ & $120.8(8)$ \\
\hline $\mathrm{C}(30)-\mathrm{C}(31)-\mathrm{C}(32)$ & $119.5(9)$ \\
\hline$C(33)-C(32)-C(31)$ & 119.1(8) \\
\hline$C(32)-C(33)-C(28)$ & $120.4(9)$ \\
\hline $\mathrm{C}(32)-\mathrm{C}(33)-\mathrm{Cl}(2)$ & $119.7(6)$ \\
\hline $\mathrm{C}(28)-\mathrm{C}(33)-\mathrm{Cl}(2)$ & $119.8(7)$ \\
\hline $\mathrm{B}\left(8^{\prime \prime}\right)-\mathrm{B}(1)-\mathrm{B}(3)$ & $106.3(6)$ \\
\hline $\mathrm{B}\left(8^{\prime \prime}\right)-\mathrm{B}(1)-\mathrm{B}(2)$ & $106.7(5)$ \\
\hline $\mathrm{B}(3)-\mathrm{B}(1)-\mathrm{B}(2)$ & $58.9(5)$ \\
\hline $\mathrm{B}\left(8^{\prime \prime}\right)-\mathrm{B}(1)-\mathrm{B}\left(18^{\prime \prime}\right)$ & $60.1(5)$ \\
\hline $\mathrm{H}(56)-\mathrm{B}(6)-\mathrm{C}(21)$ & $119(4)$ \\
\hline $\mathrm{H}(56)-\mathrm{B}(6)-\mathrm{B}(7)$ & $121(3)$ \\
\hline $\mathrm{C}(21)-\mathrm{B}(6)-\mathrm{B}(7)$ & $103.9(5)$ \\
\hline $\mathrm{H}(56)-\mathrm{B}(6)-\mathrm{B}(5)$ & $131(4)$ \\
\hline $\mathrm{C}(21)-\mathrm{B}(6)-\mathrm{B}(5)$ & $105.6(5)$ \\
\hline $\mathrm{B}(7)-\mathrm{B}(6)-\mathrm{B}(5)$ & $60.8(5)$ \\
\hline $\mathrm{H}(56)-\mathrm{B}(6)-\mathrm{B}(8)$ & $113(3)$ \\
\hline $\mathrm{C}(21)-\mathrm{B}(6)-\mathrm{B}(8)$ & $57.5(4)$ \\
\hline $\mathrm{B}(7)-\mathrm{B}(6)-\mathrm{B}(8)$ & $59.2(4)$ \\
\hline $\mathrm{B}(5)-\mathrm{B}(6)-\mathrm{B}(8)$ & $107.9(5)$ \\
\hline $\mathrm{H}(56)-\mathrm{B}(6)-\mathrm{Co}(1)$ & $116(3)$ \\
\hline $\mathrm{C}(21)-\mathrm{B}(6)-\mathrm{Co}(1)$ & $64.8(3)$ \\
\hline $\mathrm{B}(7)-\mathrm{B}(6)-\mathrm{Co}(1)$ & $118.1(5)$ \\
\hline $\mathrm{B}(5)-\mathrm{B}(6)-\mathrm{Co}(1)$ & $64.9(3)$ \\
\hline $\mathrm{B}(8)-\mathrm{B}(6)-\mathrm{Co}(1)$ & $117.0(5)$ \\
\hline $\mathrm{B}(8)-\mathrm{B}(7)-\mathrm{B}(6)$ & $61.2(5)$ \\
\hline $\mathrm{B}(8)-\mathrm{B}(7)-\mathrm{B}\left(2^{\prime \prime}\right)$ & $106.1(6)$ \\
\hline $\mathrm{B}(6)-\mathrm{B}(7)-\mathrm{B}\left(2^{\prime \prime}\right)$ & $106.9(6)$ \\
\hline $\mathrm{B}(8)-\mathrm{B}(7)-\mathrm{B}\left(1^{\prime \prime}\right)$ & $59.3(5)$ \\
\hline $\mathrm{B}(6)-\mathrm{B}(7)-\mathrm{B}\left(1^{\prime \prime}\right)$ & $108.9(7)$ \\
\hline $\mathrm{B}\left(2^{\prime \prime}\right)-\mathrm{B}(7)-\mathrm{B}\left(1^{\prime \prime}\right)$ & $59.6(5)$ \\
\hline $\mathrm{B}(8)-\mathrm{B}(7)-\mathrm{B}(5)$ & 109.1(6) \\
\hline $\mathrm{B}(6)-\mathrm{B}(7)-\mathrm{B}(5)$ & $60.3(5)$ \\
\hline
\end{tabular}




\begin{tabular}{|c|c|}
\hline $\mathrm{B}(2)-\mathrm{B}(3)-\mathrm{B}(4)$ & $59.8(5)$ \\
\hline $\mathrm{B}\left(18^{\prime \prime}\right)-\mathrm{B}(3)-\mathrm{B}(4)$ & $110.5(5)$ \\
\hline $\mathrm{B}(1)-\mathrm{B}(3)-\mathrm{B}(4)$ & $109.5(6)$ \\
\hline $\mathrm{C}(20)-\mathrm{B}(4)-\mathrm{B}(2)$ & $102.7(6)$ \\
\hline $\mathrm{C}(20)-\mathrm{B}(4)-\mathrm{B}(3)$ & $57.7(4)$ \\
\hline $\mathrm{B}(2)-\mathrm{B}(4)-\mathrm{B}(3)$ & $58.8(5)$ \\
\hline $\mathrm{C}(20)-\mathrm{B}(4)-\mathrm{B}\left(5^{\prime \prime}\right)$ & $104.7(6)$ \\
\hline $\mathrm{B}(2)-\mathrm{B}(4)-\mathrm{B}\left(5^{\prime \prime}\right)$ & $60.3(4)$ \\
\hline $\mathrm{B}(3)-\mathrm{B}(4)-\mathrm{B}\left(5^{\prime \prime}\right)$ & $107.6(6)$ \\
\hline $\mathrm{C}(20)-\mathrm{B}(4)-\mathrm{Co}(1)$ & $64.1(3)$ \\
\hline $\mathrm{B}(2)-\mathrm{B}(4)-\mathrm{Co}(1)$ & $117.5(5)$ \\
\hline $\mathrm{B}(3)-\mathrm{B}(4)-\mathrm{Co}(1)$ & $116.8(5)$ \\
\hline $\mathrm{B}\left(5^{\prime \prime}\right)-\mathrm{B}(4)-\mathrm{Co}(1)$ & $65.2(4)$ \\
\hline $\mathrm{B}\left(2^{\prime \prime}\right)-\mathrm{B}(5)-\mathrm{B}(6)$ & $105.9(6)$ \\
\hline $\mathrm{B}\left(2^{\prime \prime}\right)-\mathrm{B}(5)-\mathrm{B}\left(4^{\prime \prime}\right)$ & $59.0(4)$ \\
\hline $\mathrm{B}(6)-\mathrm{B}(5)-\mathrm{B}\left(4^{\prime \prime}\right)$ & $106.6(6)$ \\
\hline $\mathrm{B}\left(2^{\prime \prime}\right)-\mathrm{B}(5)-\mathrm{B}(7)$ & $59.7(4)$ \\
\hline $\mathrm{B}(6)-\mathrm{B}(5)-\mathrm{B}(7)$ & $58.9(5)$ \\
\hline $\mathrm{B}\left(4^{\prime \prime}\right)-\mathrm{B}(5)-\mathrm{B}(7)$ & $107.0(6)$ \\
\hline $\mathrm{B}\left(2^{\prime \prime}\right)-\mathrm{B}(5)-\mathrm{Co}(1)$ & $115.2(5)$ \\
\hline $\mathrm{B}(6)-\mathrm{B}(5)-\mathrm{Co}(1)$ & $64.3(4)$ \\
\hline $\mathrm{B}\left(4^{\prime \prime}\right)-\mathrm{B}(5)-\mathrm{Co}(1)$ & $63.7(3)$ \\
\hline $\mathrm{B}(7)-\mathrm{B}(5)-\mathrm{Co}(1)$ & $116.0(6)$ \\
\hline $\mathrm{H}(62)-\mathrm{B}(10)-\mathrm{B}\left(11^{\prime \prime \prime}\right)$ & $121(4)$ \\
\hline $\mathrm{B}(9)-\mathrm{B}(10)-\mathrm{B}\left(11^{\prime \prime \prime}\right)$ & $59.9(5)$ \\
\hline $\mathrm{H}(62)-\mathrm{B}(10)-\mathrm{B}(15)$ & $117(4)$ \\
\hline $\mathrm{B}(9)-\mathrm{B}(10)-\mathrm{B}(15)$ & $59.0(5)$ \\
\hline $\mathrm{B}\left(11^{\prime \prime \prime}\right)-\mathrm{B}(10)-\mathrm{B}(15)$ & $106.6(6)$ \\
\hline $\mathrm{H}(62)-\mathrm{B}(10)-\mathrm{B}\left(13^{\prime \prime \prime}\right)$ & $131(4)$ \\
\hline $\mathrm{B}(9)-\mathrm{B}(10)-\mathrm{B}\left(13^{\prime \prime \prime}\right)$ & $107.5(6)$ \\
\hline $\mathrm{B}\left(11^{\prime \prime \prime}\right)-\mathrm{B}(10)-\mathrm{B}\left(13^{\prime \prime \prime}\right)$ & $60.0(5)$ \\
\hline $\mathrm{B}(15)-\mathrm{B}(10)-\mathrm{B}\left(13^{\prime \prime \prime}\right)$ & $106.8(5)$ \\
\hline $\mathrm{H}(62)-\mathrm{B}(10)-\mathrm{B}(12)$ & $126(4)$ \\
\hline $\mathrm{B}(9)-\mathrm{B}(10)-\mathrm{B}(12)$ & $107.7(6)$ \\
\hline $\mathrm{B}\left(11^{\prime \prime \prime}\right)-\mathrm{B}(10)-\mathrm{B}(12)$ & $108.7(6)$ \\
\hline $\mathrm{B}(15)-\mathrm{B}(10)-\mathrm{B}(12)$ & $59.5(4)$ \\
\hline $\mathrm{B}\left(13^{\prime \prime \prime}\right)-\mathrm{B}(10)-\mathrm{B}(12)$ & $60.5(4)$ \\
\hline $\mathrm{C}(18)-\mathrm{B}(11)-\mathrm{B}\left(17^{\prime \prime \prime}\right)$ & $59.9(4)$ \\
\hline $\mathrm{C}(18)-\mathrm{B}(11)-\mathrm{B}\left(9^{\prime \prime \prime}\right)$ & $104.5(5)$ \\
\hline $\mathrm{B}\left(17^{\prime \prime \prime}\right)-\mathrm{B}(11)-\mathrm{B}\left(9^{\prime \prime \prime}\right)$ & $59.7(5)$ \\
\hline $\mathrm{C}(18)-\mathrm{B}(11)-\mathrm{B}\left(10^{\prime \prime \prime}\right)$ & $103.6(5)$ \\
\hline $\mathrm{B}\left(17^{\prime \prime \prime}\right)-\mathrm{B}(11)-\mathrm{B}\left(10^{\prime \prime \prime}\right)$ & 108.1(6) \\
\hline $\mathrm{B}\left(9^{\prime \prime \prime}\right)-\mathrm{B}(11)-\mathrm{B}\left(10^{\prime \prime \prime}\right)$ & $60.0(5)$ \\
\hline
\end{tabular}

\begin{tabular}{|c|c|}
\hline $\mathrm{B}\left(2^{\prime \prime}\right)-\mathrm{B}(7)-\mathrm{B}(5)$ & $59.7(4)$ \\
\hline $\mathrm{B}\left(1^{\prime \prime}\right)-\mathrm{B}(7)-\mathrm{B}(5)$ & $109.0(6)$ \\
\hline $\mathrm{C}(21)-\mathrm{B}(8)-\mathrm{B}\left(1^{\prime \prime}\right)$ & $106.4(6)$ \\
\hline $\mathrm{C}(21)-\mathrm{B}(8)-\mathrm{B}(7)$ & $104.8(6)$ \\
\hline $\mathrm{B}\left(1^{\prime \prime}\right)-\mathrm{B}(8)-\mathrm{B}(7)$ & $61.2(6)$ \\
\hline $\mathrm{C}(21)-\mathrm{B}(8)-\mathrm{B}(18)$ & $60.6(4)$ \\
\hline $\mathrm{B}\left(1^{\prime \prime}\right)-\mathrm{B}(8)-\mathrm{B}(18)$ & $60.6(5)$ \\
\hline $\mathrm{B}(7)-\mathrm{B}(8)-\mathrm{B}(18)$ & $110.0(6)$ \\
\hline $\mathrm{C}(21)-\mathrm{B}(8)-\mathrm{B}(6)$ & $58.3(4)$ \\
\hline $\mathrm{B}\left(1^{\prime \prime}\right)-\mathrm{B}(8)-\mathrm{B}(6)$ & $109.2(6)$ \\
\hline $\mathrm{B}(7)-\mathrm{B}(8)-\mathrm{B}(6)$ & $59.6(5)$ \\
\hline $\mathrm{B}(18)-\mathrm{B}(8)-\mathrm{B}(6)$ & $109.8(5)$ \\
\hline $\mathrm{B}(15)-\mathrm{B}(9)-\mathrm{B}(17)$ & $107.7(6)$ \\
\hline $\mathrm{B}(15)-\mathrm{B}(9)-\mathrm{B}(16)$ & $60.1(5)$ \\
\hline $\mathrm{B}(17)-\mathrm{B}(9)-\mathrm{B}(16)$ & $59.1(5)$ \\
\hline $3(15)-\mathrm{B}(9)-\mathrm{B}\left(11^{\prime \prime \prime}\right)$ & $108.3(5)$ \\
\hline $3(17)-B(9)-B\left(11^{\prime \prime \prime}\right)$ & $59.9(5)$ \\
\hline $3(16)-\mathrm{B}(9)-\mathrm{B}\left(11^{\prime \prime \prime}\right)$ & $107.1(6)$ \\
\hline $\mathrm{B}(15)-\mathrm{B}(9)-\mathrm{B}(10)$ & $60.8(5)$ \\
\hline $\mathrm{B}(17)-\mathrm{B}(9)-\mathrm{B}(10)$ & $108.4(6)$ \\
\hline $\mathrm{B}(16)-\mathrm{B}(9)-\mathrm{B}(10)$ & $108.6(6)$ \\
\hline $3\left(11^{\prime \prime \prime}\right)-\mathrm{B}(9)-\mathrm{B}(10)$ & $60.1(4)$ \\
\hline $\mathrm{H}(62)-\mathrm{B}(10)-\mathrm{B}(9)$ & $113(3)$ \\
\hline $\mathrm{C}(19)-\mathrm{B}(14)-\mathrm{B}(12)$ & $102.9(6)$ \\
\hline $\mathrm{B}(15)-\mathrm{B}(14)-\mathrm{B}(12)$ & $59.6(4)$ \\
\hline $\mathrm{B}(16)-\mathrm{B}(14)-\mathrm{B}(12)$ & $107.0(5)$ \\
\hline $\mathrm{C}(19)-\mathrm{B}(14)-\mathrm{Co}(2)$ & $63.6(3)$ \\
\hline $\mathrm{B}(15)-\mathrm{B}(14)-\mathrm{Co}(2)$ & $116.4(5)$ \\
\hline $\mathrm{B}(16)-\mathrm{B}(14)-\mathrm{Co}(2)$ & $116.4(6)$ \\
\hline $\mathrm{B}(12)-\mathrm{B}(14)-\mathrm{Co}(2)$ & $64.3(4)$ \\
\hline $\mathrm{B}(9)-\mathrm{B}(15)-\mathrm{B}(16)$ & $60.4(5)$ \\
\hline $\mathrm{B}(9)-\mathrm{B}(15)-\mathrm{B}(14)$ & $110.2(7)$ \\
\hline $\mathrm{B}(16)-\mathrm{B}(15)-\mathrm{B}(14)$ & $60.8(5)$ \\
\hline $\mathrm{B}(9)-\mathrm{B}(15)-\mathrm{B}(12)$ & $109.8(6)$ \\
\hline $\mathrm{B}(16)-\mathrm{B}(15)-\mathrm{B}(12)$ & $109.2(6)$ \\
\hline $\mathrm{B}(14)-\mathrm{B}(15)-\mathrm{B}(12)$ & $60.8(4)$ \\
\hline $\mathrm{B}(9)-\mathrm{B}(15)-\mathrm{B}(10)$ & $60.2(4)$ \\
\hline $\mathrm{B}(16)-\mathrm{B}(15)-\mathrm{B}(10)$ & $108.4(6)$ \\
\hline $\mathrm{B}(14)-\mathrm{B}(15)-\mathrm{B}(10)$ & $109.7(6)$ \\
\hline $\mathrm{B}(12)-\mathrm{B}(15)-\mathrm{B}(10)$ & $60.8(4)$ \\
\hline $\mathrm{C}(19)-\mathrm{B}(16)-\mathrm{B}(17)$ & $60.3(5)$ \\
\hline $\mathrm{C}(19)-\mathrm{B}(16)-\mathrm{B}(15)$ & $103.7(6)$ \\
\hline $\mathrm{B}(17)-\mathrm{B}(16)-\mathrm{B}(15)$ & $108.1(6)$ \\
\hline
\end{tabular}




\begin{tabular}{|c|c|c|c|}
\hline $\mathrm{C}(18)-\mathrm{B}(11)-\mathrm{B}(13)$ & $56.8(4)$ & $\mathrm{C}(19)-\mathrm{B}(16)-\mathrm{B}(9)$ & $105.2(6)$ \\
\hline $\mathrm{B}\left(17^{\prime \prime \prime}\right)-\mathrm{B}(11)-\mathrm{B}(13)$ & $108.0(5)$ & $\mathrm{B}(17)-\mathrm{B}(16)-\mathrm{B}(9)$ & $60.3(5)$ \\
\hline $\mathrm{B}\left(9^{\prime \prime \prime}\right)-\mathrm{B}(11)-\mathrm{B}(13)$ & $107.9(6)$ & $\mathrm{B}(15)-\mathrm{B}(16)-\mathrm{B}(9)$ & $59.5(5)$ \\
\hline $\mathrm{B}\left(10^{\prime \prime \prime}\right)-\mathrm{B}(11)-\mathrm{B}(13)$ & $60.4(5)$ & $\mathrm{C}(19)-\mathrm{B}(16)-\mathrm{B}(14)$ & $58.8(5)$ \\
\hline $\mathrm{B}(15)-\mathrm{B}(12)-\mathrm{B}(14)$ & $59.5(4)$ & $\mathrm{B}(17)-\mathrm{B}(16)-\mathrm{B}(14)$ & $110.5(6)$ \\
\hline $\mathrm{B}(15)-\mathrm{B}(12)-\mathrm{B}(10)$ & $59.7(4)$ & $\mathrm{B}(15)-\mathrm{B}(16)-\mathrm{B}(14)$ & $60.0(5)$ \\
\hline $\mathrm{B}(14)-\mathrm{B}(12)-\mathrm{B}(10)$ & $107.8(6)$ & $\mathrm{B}(9)-\mathrm{B}(16)-\mathrm{B}(14)$ & $108.8(6)$ \\
\hline $\mathrm{B}(15)-\mathrm{B}(12)-\mathrm{B}\left(13^{\prime \prime}\right)$ & $106.0(6)$ & C(19)-B(17)-C(18"') & $55.3(4)$ \\
\hline B(14)-B(12)-B(13"') & $106.9(6)$ & $\mathrm{C}(19)-\mathrm{B}(17)-\mathrm{B}(16)$ & $58.5(5)$ \\
\hline B(10)-B(12)-B(13"') & $59.4(4)$ & $\mathrm{C}\left(18^{\prime \prime \prime}\right)-\mathrm{B}(17)-\mathrm{B}(16)$ & $103.2(6)$ \\
\hline $\mathrm{B}(15)-\mathrm{B}(12)-\mathrm{Co}(2)$ & $116.5(5)$ & $\mathrm{C}(19)-\mathrm{B}(17)-\mathrm{B}(9)$ & $104.2(7)$ \\
\hline $\mathrm{B}(14)-\mathrm{B}(12)-\mathrm{Co}(2)$ & $64.5(4)$ & $\mathrm{C}\left(18^{\prime \prime \prime}\right)-\mathrm{B}(17)-\mathrm{B}(9)$ & $103.7(6)$ \\
\hline $\mathrm{B}(10)-\mathrm{B}(12)-\mathrm{Co}(2)$ & $117.1(5)$ & $\mathrm{B}(16)-\mathrm{B}(17)-\mathrm{B}(9)$ & $60.7(5)$ \\
\hline $\mathrm{B}\left(13^{\prime \prime \prime}\right)-\mathrm{B}(12)-\mathrm{Co}(2)$ & $64.2(4)$ & C(19)-B(17)-B(11"') & $103.4(5)$ \\
\hline $\mathrm{C}(18)-\mathrm{B}(13)-\mathrm{B}(11)$ & $59.1(4)$ & $\mathrm{C}\left(18^{\prime \prime \prime}\right)-\mathrm{B}(17)-\mathrm{B}\left(11^{\prime \prime \prime}\right)$ & $58.3(4)$ \\
\hline $\mathrm{C}(18)-\mathrm{B}(13)-\mathrm{B}\left(10^{\prime \prime \prime}\right)$ & $104.6(5)$ & B(16)-B(17)-B(11"') & $108.8(7)$ \\
\hline B(11)-B(13)-B(10"') & $59.6(4)$ & B(9)-B(17)-B(11"' ) & $60.4(5)$ \\
\hline C(18)-B(13)-B(12"' ) & $104.8(5)$ & $\mathrm{C}\left(20^{\prime \prime}\right)-\mathrm{B}(18)-\mathrm{C}(21)$ & $55.9(4)$ \\
\hline B(11)-B(13)-B(12"') & $108.0(5)$ & $\mathrm{C}\left(20^{\prime \prime}\right)-\mathrm{B}(18)-\mathrm{B}\left(3^{\prime \prime}\right)$ & $57.8(4)$ \\
\hline $\mathrm{B}\left(10^{\prime \prime \prime}\right)-\mathrm{B}(13)-\mathrm{B}\left(12^{\prime \prime \prime}\right)$ & $60.1(4)$ & $\mathrm{C}(21)-\mathrm{B}(18)-\mathrm{B}\left(3^{\prime \prime}\right)$ & $102.4(5)$ \\
\hline $\mathrm{C}(18)-\mathrm{B}(13)-\mathrm{Co}(2)$ & $65.1(3)$ & $\mathrm{C}\left(20^{\prime \prime}\right)-\mathrm{B}(18)-\mathrm{B}(8)$ & $102.0(5)$ \\
\hline $\mathrm{B}(11)-\mathrm{B}(13)-\mathrm{Co}(2)$ & $118.9(5)$ & $\mathrm{C}(21)-\mathrm{B}(18)-\mathrm{B}(8)$ & $57.2(4)$ \\
\hline $\mathrm{B}\left(10^{\prime \prime \prime}\right)-\mathrm{B}(13)-\mathrm{Co}(2)$ & $117.7(5)$ & $\mathrm{B}\left(3^{\prime \prime}\right)-\mathrm{B}(18)-\mathrm{B}(8)$ & $106.2(6)$ \\
\hline $\mathrm{B}\left(12^{\prime \prime \prime}\right)-\mathrm{B}(13)-\mathrm{Co}(2)$ & $64.2(3)$ & $\mathrm{C}\left(20^{\prime \prime}\right)-\mathrm{B}(18)-\mathrm{B}\left(1^{\prime \prime}\right)$ & $103.5(6)$ \\
\hline $\mathrm{C}(19)-\mathrm{B}(14)-\mathrm{B}(15)$ & $102.2(6)$ & $\mathrm{C}(21)-\mathrm{B}(18)-\mathrm{B}\left(1^{\prime \prime}\right)$ & $102.9(6)$ \\
\hline $\mathrm{C}(19)-\mathrm{B}(14)-\mathrm{B}(16)$ & $57.7(5)$ & $\mathrm{B}\left(3^{\prime \prime}\right)-\mathrm{B}(18)-\mathrm{B}\left(1^{\prime \prime}\right)$ & $60.0(5)$ \\
\hline $\mathrm{B}(15)-\mathrm{B}(14)-\mathrm{B}(16)$ & $59.2(5)$ & $\mathrm{B}(8)-\mathrm{B}(18)-\mathrm{B}\left(1^{\prime \prime}\right)$ & $59.3(5)$ \\
\hline
\end{tabular}

Primed labels refer to atoms generated by the following symmetry transformations: single prime: $\mathrm{x},-\mathrm{y}+3 / 2, \mathrm{z}$; double prime: $-\mathrm{x}+1,-\mathrm{y}+2, \mathrm{z}$; triple prime $-\mathrm{x},-\mathrm{y}+2, \mathrm{z}$ 
Table S24. Anisotropic displacement parameters $\left(\AA^{2} \times 10^{3}\right)$ for $\left[\mathrm{L}^{\Delta \Lambda} \mathrm{Fe}^{\mathrm{II}}\left(\mathrm{NCCH}_{3}\right)\right]$ $\left[\left(\mathrm{C}_{2} \mathrm{~B}_{9} \mathrm{H}_{11}\right)_{2} \mathrm{Co}\right]_{2} \cdot 2 \mathrm{CH}_{3} \mathrm{CN} \cdot 2 o-\mathrm{C}_{6} \mathrm{H}_{4} \mathrm{Cl}_{2}$

\begin{tabular}{|c|c|c|c|c|c|c|}
\hline Atom & $\mathrm{U}_{11}$ & $\mathrm{U}_{22}$ & $\mathrm{U}_{33}$ & $\mathrm{U}_{23}$ & $\mathrm{U}_{13}$ & $\mathrm{U}_{12}$ \\
\hline $\mathrm{Fe}(1)$ & $18(1)$ & $16(1)$ & $17(1)$ & 0 & $1(1)$ & 0 \\
\hline $\operatorname{Co}(1)$ & $19(1)$ & $19(1)$ & $20(1)$ & 0 & 0 & $-3(1)$ \\
\hline $\operatorname{Co}(2)$ & $18(1)$ & $17(1)$ & $20(1)$ & 0 & 0 & $-1(1)$ \\
\hline $\mathrm{N}(1)$ & $24(3)$ & $11(3)$ & $14(3)$ & $2(2)$ & $-6(2)$ & $-1(2)$ \\
\hline $\mathrm{N}(2)$ & $21(3)$ & $21(3)$ & $19(3)$ & $0(2)$ & $0(2)$ & $0(2)$ \\
\hline $\mathrm{N}(3)$ & $28(4)$ & $20(3)$ & $22(5)$ & 0 & $7(4)$ & 0 \\
\hline $\mathrm{N}(4)$ & $59(7)$ & $35(5)$ & $60(9)$ & 0 & $-1(6)$ & 0 \\
\hline $\mathrm{N}(5)$ & $170(20)$ & $111(13)$ & $390(40)$ & 0 & $-230(30)$ & 0 \\
\hline $\mathrm{C}(1)$ & $34(3)$ & $12(3)$ & $17(4)$ & $3(3)$ & $2(3)$ & $1(2)$ \\
\hline $\mathrm{C}(2)$ & $43(4)$ & $19(4)$ & $32(5)$ & $0(3)$ & $14(3)$ & $-6(3)$ \\
\hline$C(3)$ & $41(4)$ & $22(3)$ & $36(4)$ & $6(3)$ & $13(4)$ & $-2(3)$ \\
\hline$C(4)$ & $28(3)$ & $18(3)$ & $17(4)$ & $2(3)$ & $-1(3)$ & $6(3)$ \\
\hline$C(5)$ & $25(3)$ & $18(3)$ & $22(3)$ & $6(3)$ & $-3(3)$ & $-2(2)$ \\
\hline$C(6)$ & $30(3)$ & $27(3)$ & $29(4)$ & $0(4)$ & $3(4)$ & $5(2)$ \\
\hline$C(7)$ & $40(4)$ & $14(3)$ & $44(5)$ & $-3(3)$ & $4(4)$ & $-11(3)$ \\
\hline $\mathrm{C}(8)$ & $24(3)$ & $19(3)$ & $16(3)$ & $-5(3)$ & $5(3)$ & $-4(3)$ \\
\hline $\mathrm{C}(9)$ & $38(4)$ & $23(3)$ & $37(5)$ & $-1(3)$ & $-7(4)$ & $-11(3)$ \\
\hline$C(10)$ & $35(4)$ & $36(4)$ & $25(4)$ & $-10(4)$ & $-18(3)$ & 1(3) \\
\hline $\mathrm{C}(11)$ & $34(4)$ & $22(3)$ & $12(3)$ & $0(3)$ & $0(3)$ & $4(3)$ \\
\hline$C(12)$ & $50(6)$ & $22(5)$ & $17(5)$ & 0 & $-8(5)$ & 0 \\
\hline $\mathrm{C}(13)$ & $30(6)$ & $30(6)$ & $38(7)$ & 0 & $20(5)$ & 0 \\
\hline $\mathrm{C}(14)$ & $88(9)$ & $29(5)$ & $13(6)$ & 0 & $4(6)$ & 0 \\
\hline $\mathrm{C}(15)$ & $52(7)$ & $15(5)$ & $21(5)$ & 0 & $-3(5)$ & 0 \\
\hline$C(16)$ & $26(5)$ & $24(5)$ & $32(6)$ & 0 & $-17(5)$ & 0 \\
\hline $\mathrm{C}(17)$ & $49(7)$ & $31(5)$ & $31(7)$ & 0 & $-4(5)$ & 0 \\
\hline $\mathrm{C}(18)$ & $26(4)$ & $24(3)$ & $21(4)$ & $-2(3)$ & $3(3)$ & $1(3)$ \\
\hline$C(19)$ & $26(3)$ & $19(3)$ & $32(4)$ & $0(3)$ & $4(4)$ & $-1(3)$ \\
\hline $\mathrm{C}(20)$ & $24(3)$ & $23(3)$ & $29(4)$ & $3(3)$ & $-4(3)$ & $0(3)$ \\
\hline $\mathrm{C}(21)$ & $28(4)$ & $30(4)$ & $21(4)$ & $0(3)$ & $-5(3)$ & $-5(3)$ \\
\hline $\mathrm{C}(22)$ & $35(6)$ & $13(4)$ & $21(6)$ & 0 & $-2(4)$ & 0 \\
\hline $\mathrm{C}(23)$ & $27(5)$ & $26(4)$ & $43(7)$ & 0 & $-9(5)$ & 0 \\
\hline $\mathrm{C}(24)$ & $21(5)$ & $34(5)$ & $50(7)$ & 0 & $4(6)$ & 0 \\
\hline $\mathrm{C}(25)$ & $83(9)$ & $34(6)$ & $84(12)$ & 0 & $-46(9)$ & 0 \\
\hline$C(26)$ & $60(9)$ & $69(10)$ & $170(20)$ & 0 & $-55(13)$ & 0 \\
\hline $\mathrm{C}(27)$ & $70(9)$ & $46(7)$ & $87(13)$ & 0 & $-2(9)$ & 0 \\
\hline $\mathrm{C}(28)$ & $36(4)$ & $39(4)$ & $60(6)$ & $-11(4)$ & $-4(4)$ & $4(3)$ \\
\hline
\end{tabular}




\begin{tabular}{rrrrrrr}
$\mathrm{C}(29)$ & $32(4)$ & $57(5)$ & $71(7)$ & $-20(5)$ & $10(4)$ & $-10(4)$ \\
$\mathrm{C}(30)$ & $39(4)$ & $37(4)$ & $39(5)$ & $-6(4)$ & $13(4)$ & $5(3)$ \\
$\mathrm{C}(31)$ & $63(6)$ & $44(5)$ & $47(6)$ & $-17(4)$ & $-13(5)$ & $1(4)$ \\
$\mathrm{C}(32)$ & $58(5)$ & $38(4)$ & $44(5)$ & $-20(4)$ & $-13(5)$ & $3(4)$ \\
$\mathrm{C}(33)$ & $37(4)$ & $42(4)$ & $48(5)$ & $-15(4)$ & $4(4)$ & $-1(3)$ \\
$\mathrm{B}(1)$ & $34(4)$ & $19(3)$ & $40(5)$ & $-2(4)$ & $-4(4)$ & $-4(3)$ \\
$\mathrm{B}(2)$ & $31(4)$ & $26(4)$ & $26(5)$ & $0(4)$ & $1(4)$ & $-7(3)$ \\
$\mathrm{B}(3)$ & $25(4)$ & $24(4)$ & $36(5)$ & $3(4)$ & $3(4)$ & $-11(3)$ \\
$\mathrm{B}(4)$ & $24(4)$ & $23(4)$ & $21(5)$ & $2(3)$ & $7(3)$ & $-6(3)$ \\
$\mathrm{B}(5)$ & $17(4)$ & $21(4)$ & $35(5)$ & $0(4)$ & $0(4)$ & $3(3)$ \\
$\mathrm{B}(6)$ & $26(4)$ & $21(3)$ & $28(5)$ & $1(4)$ & $-10(4)$ & $1(3)$ \\
$\mathrm{B}(7)$ & $32(5)$ & $23(4)$ & $30(5)$ & $4(4)$ & $-5(4)$ & $0(3)$ \\
$\mathrm{B}(8)$ & $28(4)$ & $30(4)$ & $34(5)$ & $3(4)$ & $-1(4)$ & $-1(3)$ \\
$\mathrm{B}(9)$ & $26(4)$ & $22(4)$ & $44(6)$ & $1(4)$ & $6(5)$ & $-1(3)$ \\
$\mathrm{B}(10)$ & $29(4)$ & $19(4)$ & $25(5)$ & $1(4)$ & $-5(4)$ & $-4(3)$ \\
$\mathrm{B}(11)$ & $24(4)$ & $19(4)$ & $35(6)$ & $7(4)$ & $2(4)$ & $-4(3)$ \\
$\mathrm{B}(12)$ & $23(4)$ & $15(3)$ & $24(5)$ & $0(3)$ & $-7(4)$ & $-4(3)$ \\
$\mathrm{B}(13)$ & $22(4)$ & $24(4)$ & $25(4)$ & $5(4)$ & $7(4)$ & $1(3)$ \\
$\mathrm{B}(14)$ & $21(4)$ & $22(4)$ & $30(5)$ & $5(3)$ & $-3(4)$ & $0(3)$ \\
$\mathrm{B}(15)$ & $29(4)$ & $17(4)$ & $30(5)$ & $0(4)$ & $-7(4)$ & $-2(3)$ \\
$\mathrm{B}(16)$ & $26(4)$ & $24(4)$ & $45(6)$ & $-1(4)$ & $1(4)$ & $-1(3)$ \\
$\mathrm{B}(17)$ & $33(4)$ & $21(4)$ & $39(6)$ & $-5(4)$ & $4(4)$ & $0(3)$ \\
$\mathrm{B}(18)$ & $38(5)$ & $20(4)$ & $31(5)$ & $11(4)$ & $-11(4)$ & $-6(3)$ \\
$\mathrm{Cl}(1)$ & $53(1)$ & $70(1)$ & $90(2)$ & $19(1)$ & $-13(1)$ & $2(1)$ \\
$\mathrm{Cl}(2)$ & $49(1)$ & $83(2)$ & $91(2)$ & $23(2)$ & $18(2)$ & $-4(1)$ \\
\hline & & & & & &
\end{tabular}

The anisotropic displacement factor exponent takes the form: $-2 \pi^{2} h^{2} a^{* 2} U_{11}+\ldots+$ $\left.2 \mathrm{hka} * \mathrm{~b} * \mathrm{U}_{12}\right]$ 
Table S25. Hydrogen coordinates $\left(\times 10^{4}\right)$ and isotropic displacement parameters $\left(\AA^{2} \times 10^{3}\right)$ for $\left[\mathrm{L}^{\Delta \Delta} \mathrm{Fe}^{\mathrm{II}}\left(\mathrm{NCCH}_{3}\right)\right]\left[\left(\mathrm{C}_{2} \mathrm{~B}_{9} \mathrm{H}_{11}\right)_{2} \mathrm{Co}\right]_{2} \cdot 2$ $\mathrm{CH}_{3} \mathrm{CN} \cdot 2 o-\mathrm{C}_{6} \mathrm{H}_{4} \mathrm{Cl}_{2}$.

\begin{tabular}{|c|c|c|c|c|}
\hline Atom & $\mathrm{x}$ & $\mathrm{y}$ & z & $\mathrm{U}(\mathrm{eq})$ \\
\hline $\mathrm{H}(2)$ & 7071 & 6910 & 12257 & 38 \\
\hline $\mathrm{H}(3)$ & 6908 & 6187 & 11063 & 39 \\
\hline $\mathrm{H}(6 \mathrm{~A})$ & 8469 & 6292 & 9032 & 43 \\
\hline $\mathrm{H}(6 \mathrm{~B})$ & 8231 & 5758 & 8529 & 43 \\
\hline $\mathrm{H}(6 \mathrm{C})$ & 8303 & 5795 & 9725 & 43 \\
\hline $\mathrm{H}(7 \mathrm{~A})$ & 7237 & 5494 & 8703 & 49 \\
\hline $\mathrm{H}(7 \mathrm{~B})$ & 6783 & 5857 & 9286 & 49 \\
\hline $\mathrm{H}(7 \mathrm{C})$ & 7255 & 5533 & 9905 & 49 \\
\hline $\mathrm{H}(9)$ & 6738 & 6174 & 7554 & 39 \\
\hline $\mathrm{H}(10)$ & 6780 & 6899 & 6314 & 38 \\
\hline $\mathrm{H}(13 \mathrm{~A})$ & 8691 & 7216 & 6164 & 49 \\
\hline $\mathrm{H}(13 \mathrm{~B})$ & 8614 & 7441 & 7282 & 49 \\
\hline $\mathrm{H}(13 \mathrm{C})$ & 8678 & 7844 & 6357 & 49 \\
\hline $\mathrm{H}(30 \mathrm{~A})$ & 7281 & 7469 & 5297 & 65 \\
\hline $\mathrm{H}(31 \mathrm{~B})$ & 7875 & 7199 & 4993 & 65 \\
\hline $\mathrm{H}(32 \mathrm{C})$ & 7819 & 7833 & 5022 & 65 \\
\hline $\mathrm{H}(16 \mathrm{~A})$ & 8790 & 7471 & 10743 & 41 \\
\hline $\mathrm{H}(16 \mathrm{~B})$ & 8973 & 7197 & 11782 & 41 \\
\hline $\mathrm{H}(16 \mathrm{C})$ & 8957 & 7832 & 11690 & 41 \\
\hline $\mathrm{H}(17 \mathrm{~A})$ & 8046 & 7136 & 13181 & 55 \\
\hline $\mathrm{H}(17 \mathrm{~B})$ & 7787 & 7724 & 13146 & 55 \\
\hline $\mathrm{H}(17 \mathrm{C})$ & 8462 & 7640 & 13237 & 55 \\
\hline $\mathrm{H}(23 \mathrm{~A})$ & 10233 & 7139 & 8741 & 48 \\
\hline $\mathrm{H}(23 \mathrm{~B})$ & 10239 & 7620 & 9531 & 48 \\
\hline $\mathrm{H}(23 \mathrm{C})$ & 10230 & 7741 & 8349 & 48 \\
\hline $\mathrm{H}(24 \mathrm{~A})$ & 5481 & 7372 & 9213 & 101 \\
\hline $\mathrm{H}(25 \mathrm{~B})$ & 5703 & 7265 & 8092 & 101 \\
\hline $\mathrm{H}(26 \mathrm{C})$ & 5625 & 7862 & 8487 & 101 \\
\hline $\mathrm{H}(27 \mathrm{~A})$ & 5630 & 7133 & 2806 & 102 \\
\hline $\mathrm{H}(27 \mathrm{~B})$ & 5925 & 7667 & 2407 & 102 \\
\hline $\mathrm{H}(27 \mathrm{C})$ & 5384 & 7700 & 3140 & 102 \\
\hline $\mathrm{H}(29)$ & 8457 & 9625 & 2827 & 64 \\
\hline $\mathrm{H}(30)$ & 7971 & 10078 & 1495 & 46 \\
\hline $\mathrm{H}(31)$ & 7013 & 10091 & 1438 & 62 \\
\hline $\mathrm{H}(32)$ & 6469 & 9568 & 2642 & 56 \\
\hline $\mathrm{H}(50)$ & $4990(30)$ & $7950(30)$ & $880(80)$ & $50(20)$ \\
\hline
\end{tabular}




$\begin{array}{lrrrr}\mathrm{H}(51) & 4650(30) & 8670(20) & 2700(50) & 18(16) \\ \mathrm{H}(52) & 3990(30) & 8710(30) & 820(70) & 50(20) \\ \mathrm{H}(53) & 4120(20) & 9570(20) & 1840(40) & 0(13) \\ \mathrm{H}(54) & 4260(20) & 9530(20) & -160(50) & 16(16) \\ \mathrm{H}(55) & 4670(30) & 10380(30) & 2770(50) & 31(19) \\ \mathrm{H}(56) & 3900(20) & 10410(20) & 750(50) & 30(18) \\ \mathrm{H}(57) & 4730(30) & 10480(30) & -740(60) & 40(20) \\ \mathrm{H}(58) & 4090(30) & 11330(20) & 1980(50) & 20(16) \\ \mathrm{H}(59) & 4200(30) & 11360(30) & -280(60) & 50(20) \\ \mathrm{H}(60) & 5360(20) & 11340(20) & -890(50) & 21(15) \\ \mathrm{H}(61) & 170(40) & 8000(30) & 1410(80) & 70(30) \\ \mathrm{H}(62) & -680(30) & 8520(20) & 2500(50) & 28(17) \\ \mathrm{H}(63) & 640(20) & 11460(20) & 230(50) & 18(16) \\ \mathrm{H}(64) & -360(20) & 9580(20) & 3190(40) & 5(13) \\ \mathrm{H}(65) & 1070(30) & 10550(40) & 1220(80) & 80(30) \\ \mathrm{H}(66) & 170(20) & 10480(20) & -140(40) & 0(13) \\ \mathrm{H}(67) & 940(30) & 9640(30) & 2510(50) & 33(19) \\ \mathrm{H}(68) & 830(20) & 9589(19) & 310(40) & 3(13) \\ \mathrm{H}(69) & 470(20) & 8700(20) & 3210(50) & 13(15) \\ \mathrm{H}(70) & 1210(30) & 8770(30) & 1420(60) & 36(19) \\ \mathrm{H}(71) & 500(30) & 8750(30) & -360(60) & 60(30) \\ & & & & \end{array}$


Table S26. Computed frontier orbital occupancies and energies (in eV) for each spin of $\left[\mathrm{LFe}^{\mathrm{II}}\right]^{2-}$.

\begin{tabular}{cccccc}
\hline & $\alpha$ & & \multicolumn{3}{c}{$\beta$} \\
Orb. & Occup. & Energy & Orb. & Occup. & Energy \\
\hline 83 & 1 & 0.251 & 81 & 1 & 0.322 \\
84 & 1 & 0.915 & 82 & 1 & 0.556 \\
85 & 1 & 1.347 & 83 & 1 & 0.562 \\
86 & 1 & 1.421 & 84 & 1 & 0.931 \\
87 & 1 & 1.497 & 85 & 1 & 1.369 \\
88 & 1 & 1.639 & 86 & 1 & 1.448 \\
89 & 1 & 1.77 & 87 & 1 & 1.529 \\
90 & 1 & 1.787 & 88 & 1 & 1.665 \\
91 & 1 & 2.049 & 89 & 1 & 3.032 \\
92 & 1 & 2.253 & 90 & 1 & 3.056 \\
93 & 0 & 5.305 & 91 & 0 & 4.132 \\
94 & 0 & 5.943 & 92 & 0 & 4.161 \\
95 & 0 & 6.077 & 93 & 0 & 6.011 \\
96 & 0 & 6.396 & 94 & 0 & 6.09 \\
97 & 0 & 6.403 & 95 & 0 & 6.294 \\
98 & 0 & 6.428 & 96 & 0 & 6.401 \\
99 & 0 & 6.728 & 97 & 0 & 6.408 \\
100 & 0 & 6.781 & 98 & 0 & 6.434 \\
101 & 0 & 6.973 & 0 & 0 & 6.861 \\
102 & 0 & 7.152 & & & \\
\hline & & & 99 & 0 & \\
\hline
\end{tabular}




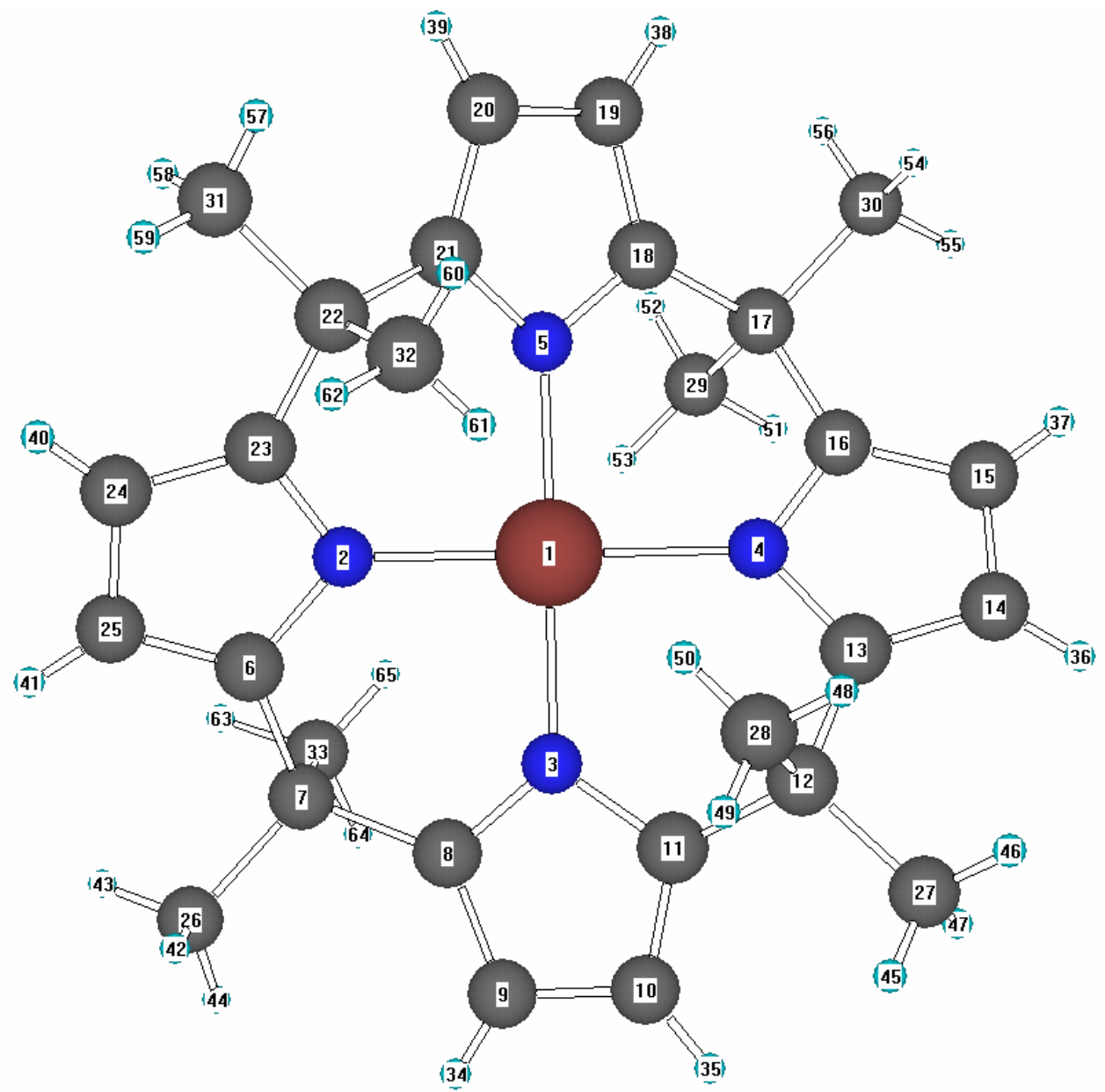

Figure S7. Atom labeling scheme for DFT calculations of $\left[\mathrm{LFe}^{\mathrm{II}}\right]^{2-}$. 
Table S27. Mulliken population analysis for $\left[\mathrm{LFe}^{\mathrm{II}}\right]^{2-}$.

\begin{tabular}{|c|c|c|c|c|c|}
\hline Atom & Charge & $\operatorname{Spin}(\times 2)$ & Atom & Charge & $\operatorname{Spin}(\times 2)$ \\
\hline $1 \mathrm{Fe}$ & 0.8242 & 1.9882 & $34 \mathrm{H}$ & -0.1966 & -0.0002 \\
\hline $2 \mathrm{~N}$ & -0.0539 & -0.0297 & $35 \mathrm{H}$ & -0.1887 & -0.0004 \\
\hline $3 \mathrm{~N}$ & -0.0822 & -0.028 & $36 \mathrm{H}$ & -0.1802 & -0.0004 \\
\hline $4 \mathrm{~N}$ & -0.057 & -0.0289 & $37 \mathrm{H}$ & -0.196 & -0.0004 \\
\hline $5 \mathrm{~N}$ & -0.0631 & -0.0291 & $38 \mathrm{H}$ & -0.1938 & -0.0004 \\
\hline $6 \mathrm{C}$ & -0.06 & 0.0084 & $39 \mathrm{H}$ & -0.1785 & -0.0003 \\
\hline $7 \mathrm{C}$ & -0.558 & -0.0016 & $40 \mathrm{H}$ & -0.1785 & -0.0004 \\
\hline $8 \mathrm{C}$ & -0.0463 & 0.0102 & $41 \mathrm{H}$ & -0.2007 & -0.0003 \\
\hline $9 \mathrm{C}$ & 0.0885 & 0.0069 & $42 \mathrm{H}$ & -0.0496 & -0.0003 \\
\hline $10 \mathrm{C}$ & 0.0605 & 0.0106 & $43 \mathrm{H}$ & -0.0717 & 0 \\
\hline $11 \mathrm{C}$ & -0.0318 & 0.0072 & $44 \mathrm{H}$ & -0.0834 & 0 \\
\hline $12 \mathrm{C}$ & -0.5568 & -0.0013 & $45 \mathrm{H}$ & -0.0533 & 0 \\
\hline $13 \mathrm{C}$ & -0.0348 & 0.009 & $46 \mathrm{H}$ & -0.051 & 0 \\
\hline $14 \mathrm{C}$ & 0.0485 & 0.009 & $47 \mathrm{H}$ & -0.064 & -0.0002 \\
\hline $15 \mathrm{C}$ & 0.074 & 0.0096 & $48 \mathrm{H}$ & -0.0921 & -0.0002 \\
\hline $16 \mathrm{C}$ & -0.0455 & 0.0087 & $49 \mathrm{H}$ & -0.0953 & -0.0002 \\
\hline $17 \mathrm{C}$ & -0.558 & -0.0014 & $50 \mathrm{H}$ & 0.07 & -0.0028 \\
\hline $18 \mathrm{C}$ & -0.0412 & 0.0078 & $51 \mathrm{H}$ & -0.0735 & -0.0001 \\
\hline $19 \mathrm{C}$ & 0.0793 & 0.0101 & $52 \mathrm{H}$ & -0.0852 & -0.0002 \\
\hline $20 \mathrm{C}$ & 0.0404 & 0.008 & $53 \mathrm{H}$ & 0.057 & -0.0029 \\
\hline $21 \mathrm{C}$ & -0.039 & 0.0094 & $54 \mathrm{H}$ & -0.0797 & -0.0002 \\
\hline $22 \mathrm{C}$ & -0.5702 & -0.0016 & $55 \mathrm{H}$ & -0.0555 & 0.0001 \\
\hline $23 \mathrm{C}$ & -0.0369 & 0.0091 & $56 \mathrm{H}$ & -0.063 & 0 \\
\hline $24 \mathrm{C}$ & 0.0356 & 0.0093 & $57 \mathrm{H}$ & -0.073 & 0.0001 \\
\hline $25 \mathrm{C}$ & 0.1012 & 0.0095 & $58 \mathrm{H}$ & -0.0529 & -0.0002 \\
\hline $26 \mathrm{C}$ & 0.3071 & 0 & $59 \mathrm{H}$ & -0.0703 & 0 \\
\hline $27 \mathrm{C}$ & 0.2528 & 0 & $60 \mathrm{H}$ & -0.1073 & -0.0001 \\
\hline $28 \mathrm{C}$ & 0.2737 & 0.0017 & $61 \mathrm{H}$ & 0.052 & -0.0031 \\
\hline $29 \mathrm{C}$ & 0.2526 & 0.0016 & $62 \mathrm{H}$ & -0.0911 & -0.0002 \\
\hline $30 \mathrm{C}$ & 0.2885 & 0 & $63 \mathrm{H}$ & -0.0834 & -0.0001 \\
\hline $31 \mathrm{C}$ & 0.2891 & 0 & $64 \mathrm{H}$ & -0.0683 & 0 \\
\hline $32 \mathrm{C}$ & 0.3154 & 0.0018 & $65 \mathrm{H}$ & 0.0576 & -0.0027 \\
\hline $33 \mathrm{C}$ & 0.2431 & 0.0015 & & & \\
\hline
\end{tabular}


Table S28. Computed frontier orbital occupancies and energies (in eV) for each spin of [ $\left.\mathrm{LFe}^{\mathrm{III}}\right]^{-}$.

\begin{tabular}{cccccc}
\hline \multicolumn{3}{c}{$\alpha$} & & \multicolumn{3}{c}{$\beta$} \\
Orb. & Occup. & Energy & Orb. & Occup. & Energy \\
\hline 83 & 1 & -4.112 & 80 & 1 & -4.573 \\
84 & 1 & -3.629 & 81 & 1 & -3.209 \\
85 & 1 & -2.509 & 82 & 1 & -3.053 \\
86 & 1 & -2.495 & 83 & 1 & -3.008 \\
87 & 1 & -2.4 & 84 & 1 & -2.386 \\
88 & 1 & -2.395 & 85 & 1 & -2.033 \\
89 & 1 & -2.046 & 86 & 1 & -1.898 \\
90 & 1 & -1.918 & 87 & 1 & -1.855 \\
91 & 1 & -1.871 & 88 & 1 & -1.731 \\
92 & 1 & -1.762 & 89 & 1 & -1.443 \\
93 & 0 & 0.975 & 90 & 0 & -1.245 \\
94 & 0 & 2.282 & 91 & 0 & -1.102 \\
95 & 0 & 3.21 & 92 & 0 & -0.939 \\
96 & 0 & 3.272 & 93 & 0 & 2.277 \\
97 & 0 & 3.316 & 94 & 0 & 2.545 \\
98 & 0 & 3.526 & 95 & 0 & 3.273 \\
99 & 0 & 3.734 & 96 & 0 & 3.385 \\
100 & 0 & 3.794 & 97 & 0 & 3.421 \\
101 & 0 & 3.807 & 98 & 0 & 3.628 \\
102 & 0 & 3.835 & 99 & 0 & 3.804 \\
\hline
\end{tabular}




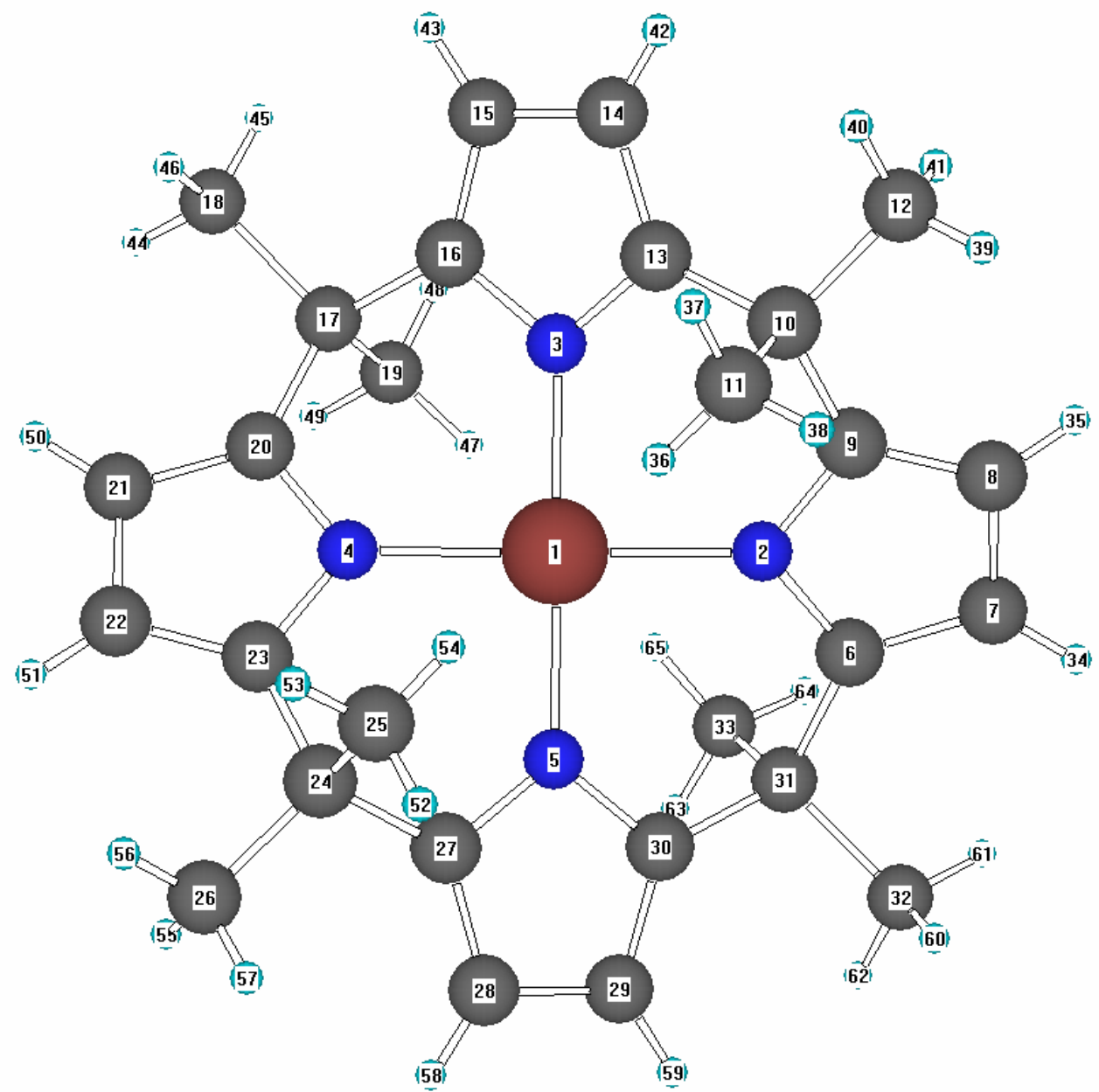

Figure S8. Atom labeling scheme for DFT calculations of [LFe $\left.{ }^{\mathrm{III}}\right]^{-}$. 
Table S29. Mulliken population analysis for $\left[\mathrm{LFe}^{\mathrm{III}}\right]^{-}$.

\begin{tabular}{|c|c|c|c|c|c|}
\hline Atom & Charge & Spin $(\times 2)$ & Atom & Charge & $\operatorname{Spin}(\times 2)$ \\
\hline $1 \mathrm{Fe}$ & 1.1348 & 2.642 & $34 \mathrm{H}$ & -0.1385 & -0.0015 \\
\hline $2 \mathrm{~N}$ & -0.0523 & 0.0221 & $35 \mathrm{H}$ & -0.1396 & -0.0013 \\
\hline $3 \mathrm{~N}$ & -0.0532 & 0.0192 & $36 \mathrm{H}$ & 0.0766 & -0.0022 \\
\hline $4 \mathrm{~N}$ & -0.0512 & 0.0207 & $37 \mathrm{H}$ & -0.0648 & 0.0003 \\
\hline $5 \mathrm{~N}$ & -0.0589 & 0.0241 & $38 \mathrm{H}$ & -0.0647 & 0.0001 \\
\hline $6 \mathrm{C}$ & -0.0305 & -0.0062 & $39 \mathrm{H}$ & -0.0347 & 0 \\
\hline $7 \mathrm{C}$ & 0.059 & 0.0433 & $40 \mathrm{H}$ & -0.0307 & 0 \\
\hline $8 \mathrm{C}$ & 0.0668 & 0.0343 & $41 \mathrm{H}$ & -0.0193 & 0 \\
\hline $9 \mathrm{C}$ & -0.0262 & 0 & $42 \mathrm{H}$ & -0.1385 & -0.0013 \\
\hline $10 \mathrm{C}$ & -0.5696 & -0.0011 & $43 \mathrm{H}$ & -0.1401 & -0.0013 \\
\hline $11 \mathrm{C}$ & 0.2336 & 0.0031 & $44 \mathrm{H}$ & -0.0335 & 0 \\
\hline $12 \mathrm{C}$ & 0.2004 & -0.0001 & $45 \mathrm{H}$ & -0.0304 & 0 \\
\hline $13 \mathrm{C}$ & -0.0297 & -0.0041 & $46 \mathrm{H}$ & -0.0197 & 0 \\
\hline $14 \mathrm{C}$ & 0.0538 & 0.0401 & $47 \mathrm{H}$ & 0.0874 & -0.0021 \\
\hline $15 \mathrm{C}$ & 0.0738 & 0.0345 & $48 \mathrm{H}$ & -0.0673 & 0.0001 \\
\hline $16 \mathrm{C}$ & -0.0262 & -0.0007 & $49 \mathrm{H}$ & -0.0626 & 0.0001 \\
\hline $17 \mathrm{C}$ & -0.568 & -0.001 & $50 \mathrm{H}$ & -0.1389 & -0.0014 \\
\hline $18 \mathrm{C}$ & 0.1981 & -0.0001 & $51 \mathrm{H}$ & -0.1391 & -0.0013 \\
\hline $19 \mathrm{C}$ & 0.2179 & 0.0031 & $52 \mathrm{H}$ & -0.0609 & 0.0002 \\
\hline $20 \mathrm{C}$ & -0.0271 & -0.0037 & $53 \mathrm{H}$ & -0.0676 & 0.0003 \\
\hline $21 \mathrm{C}$ & 0.0648 & 0.0383 & $54 \mathrm{H}$ & 0.0837 & -0.0024 \\
\hline $22 \mathrm{C}$ & 0.0614 & 0.0369 & $55 \mathrm{H}$ & -0.0211 & 0 \\
\hline $23 \mathrm{C}$ & -0.0336 & -0.0026 & $56 \mathrm{H}$ & -0.0336 & 0 \\
\hline $24 \mathrm{C}$ & -0.5667 & -0.0011 & $57 \mathrm{H}$ & -0.0312 & 0 \\
\hline $25 \mathrm{C}$ & 0.2239 & 0.0034 & $58 \mathrm{H}$ & -0.138 & -0.0013 \\
\hline $26 \mathrm{C}$ & 0.203 & -0.0001 & $59 \mathrm{H}$ & -0.1354 & -0.0015 \\
\hline $27 \mathrm{C}$ & -0.0236 & -0.0005 & $60 \mathrm{H}$ & -0.0213 & 0 \\
\hline $28 \mathrm{C}$ & 0.0599 & 0.0356 & $61 \mathrm{H}$ & -0.0294 & 0 \\
\hline $29 \mathrm{C}$ & 0.0614 & 0.0428 & $62 \mathrm{H}$ & -0.0357 & 0 \\
\hline $30 \mathrm{C}$ & -0.025 & -0.0055 & $63 \mathrm{H}$ & -0.0613 & 0.0001 \\
\hline $31 \mathrm{C}$ & -0.5686 & -0.0007 & $64 \mathrm{H}$ & -0.0672 & 0.0001 \\
\hline $32 \mathrm{C}$ & 0.2042 & -0.0002 & $65 \mathrm{H}$ & 0.0874 & -0.0021 \\
\hline $33 \mathrm{C}$ & 0.2239 & 0.0027 & & & \\
\hline
\end{tabular}


Table S30. Computed frontier orbital occupancies and energies (in eV) for each spin of $\left[\mathrm{L}^{\Delta \Delta} \mathrm{Fe}^{\mathrm{II}}\left(\mathrm{NCCH}_{3}\right)^{2+}\right.$.

\begin{tabular}{cccccc}
\hline \multicolumn{3}{c}{$\alpha$} & & \multicolumn{3}{c}{$\beta$} \\
Orb. & Occup. & Energy & Orb. & Occup. & Energy \\
\hline 90 & 1 & -13.326 & 86 & 1 & -13.880 \\
91 & 1 & -13.164 & 87 & 1 & -13.310 \\
92 & 1 & -12.979 & 88 & 1 & -13.224 \\
93 & 1 & -12.864 & 89 & 1 & -13.206 \\
94 & 1 & -12.547 & 90 & 1 & -12.972 \\
95 & 1 & -12.527 & 91 & 1 & -12.866 \\
96 & 1 & -12.484 & 92 & 1 & -12.822 \\
97 & 1 & -12.346 & 93 & 1 & -12.686 \\
98 & 1 & -12.101 & 94 & 1 & -12.477 \\
99 & 1 & -10.659 & 95 & 1 & -10.227 \\
100 & 0 & -9.362 & 96 & 0 & -9.958 \\
101 & 0 & -8.944 & 97 & 0 & -9.916 \\
102 & 0 & -8.430 & 98 & 0 & -9.497 \\
103 & 0 & -7.939 & 99 & 0 & -9.077 \\
104 & 0 & -7.385 & 100 & 0 & -8.972 \\
105 & 0 & -7.328 & 101 & 0 & -8.262 \\
106 & 0 & -6.168 & 102 & 0 & -7.789 \\
107 & 0 & -5.812 & 103 & 0 & -7.441 \\
108 & 0 & -5.526 & 104 & 0 & -7.097 \\
109 & 0 & -5.410 & 105 & 0 & -7.057 \\
\hline
\end{tabular}




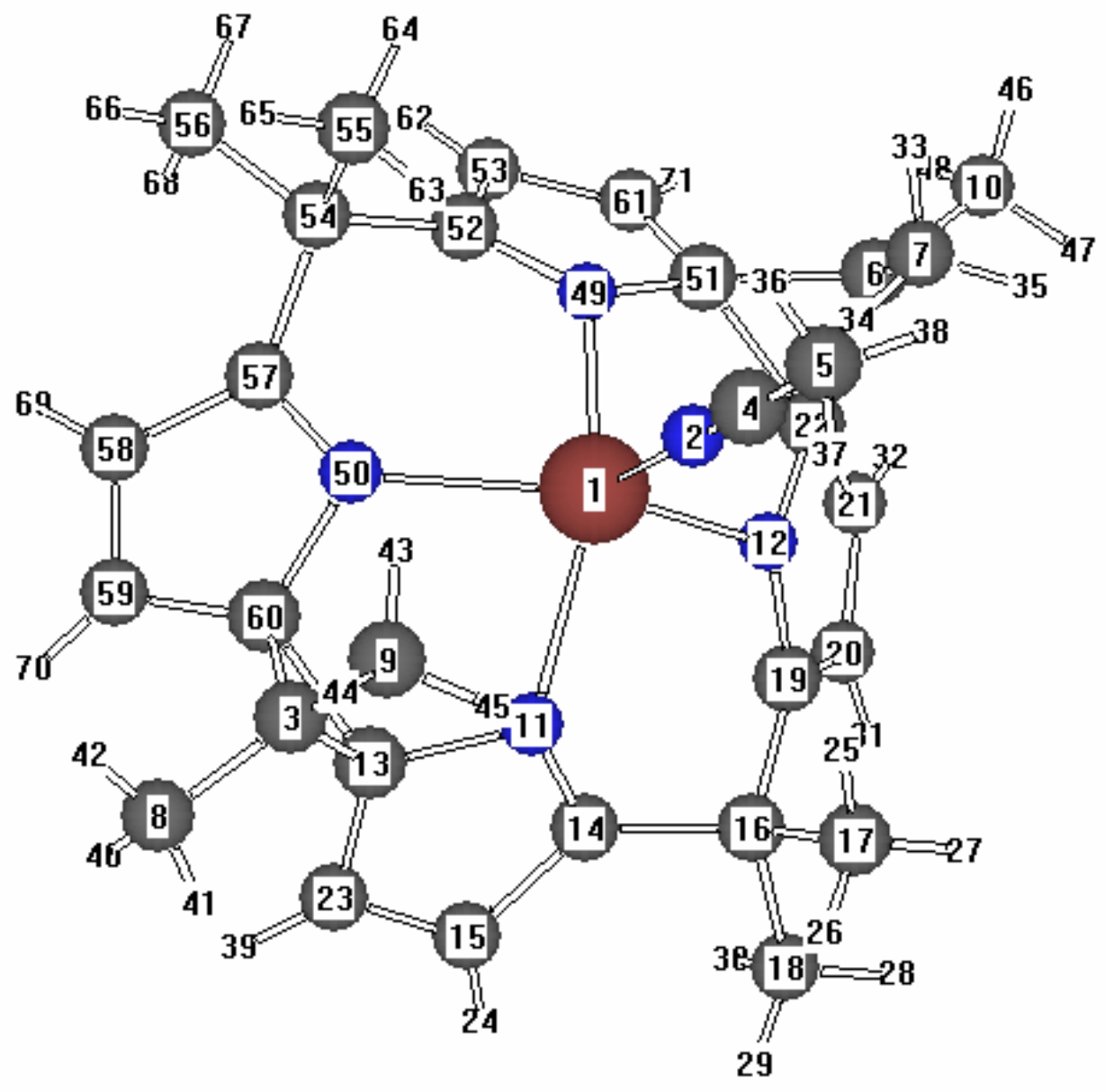

Figure S9. Atom labeling scheme for DFT calculations of $\left[\mathrm{L}^{\Delta \Lambda} \mathrm{Fe}^{\mathrm{II}}\left(\mathrm{NCCH}_{3}\right)\right]^{2+}$. 
Table S31. Mulliken population analysis for $\left[\mathrm{L}^{\Delta \Lambda} \mathrm{Fe}^{\mathrm{II}}\left(\mathrm{NCCH}_{3}\right)^{2+}\right.$.

\begin{tabular}{|c|c|c|c|c|c|}
\hline Atom & Charge & $\operatorname{Spin}(\times 2)$ & Atom & Charge & Spin $(\times 2)$ \\
\hline $1 \mathrm{Fe}$ & 0.9233 & 3.6516 & $37 \mathrm{H}$ & 0.0061 & 0.0022 \\
\hline $2 \mathrm{~N}$ & 0.0272 & -0.0269 & $38 \mathrm{H}$ & 0.0009 & 0.0023 \\
\hline $3 \mathrm{C}$ & -0.2528 & 0.0018 & $39 \mathrm{H}$ & -0.0679 & -0.001 \\
\hline $4 \mathrm{C}$ & -0.054 & 0.0671 & $40 \mathrm{H}$ & -0.0008 & 0 \\
\hline $5 \mathrm{C}$ & 0.186 & -0.0024 & $41 \mathrm{H}$ & 0.017 & -0.0001 \\
\hline $6 \mathrm{C}$ & -0.2792 & 0.0016 & $42 \mathrm{H}$ & 0.0181 & -0.0001 \\
\hline $7 \mathrm{C}$ & 0.1315 & 0.0022 & $43 \mathrm{H}$ & 0.0437 & 0.0003 \\
\hline $8 \mathrm{C}$ & 0.2132 & -0.0004 & $44 \mathrm{H}$ & -0.0211 & -0.0001 \\
\hline $9 \mathrm{C}$ & 0.1817 & -0.0001 & $45 \mathrm{H}$ & 0.0308 & 0.0003 \\
\hline $10 \mathrm{C}$ & 0.2134 & -0.0006 & $46 \mathrm{H}$ & 0.0093 & -0.0001 \\
\hline $11 \mathrm{~N}$ & -0.0505 & 0.0187 & $47 \mathrm{H}$ & 0.0151 & 0 \\
\hline $12 \mathrm{~N}$ & -0.0834 & 0.017 & $48 \mathrm{H}$ & 0.0059 & 0.0001 \\
\hline $13 \mathrm{C}$ & -0.0646 & -0.0035 & $49 \mathrm{~N}$ & -0.0393 & 0.0187 \\
\hline $14 \mathrm{C}$ & 0.048 & 0.0325 & $50 \mathrm{~N}$ & -0.0867 & 0.017 \\
\hline $15 \mathrm{C}$ & 0.1407 & -0.0059 & $51 \mathrm{C}$ & -0.0636 & -0.0035 \\
\hline $16 \mathrm{C}$ & -0.5464 & -0.0029 & $52 \mathrm{C}$ & 0.0494 & 0.0322 \\
\hline $17 \mathrm{C}$ & 0.2073 & 0.003 & $53 \mathrm{C}$ & 0.1461 & -0.006 \\
\hline $18 \mathrm{C}$ & 0.1336 & -0.0001 & $54 \mathrm{C}$ & -0.5441 & -0.0029 \\
\hline $19 \mathrm{C}$ & 0.0494 & 0.0378 & $55 \mathrm{C}$ & 0.2067 & 0.0031 \\
\hline $20 \mathrm{C}$ & 0.1437 & -0.0092 & $56 \mathrm{C}$ & 0.1334 & -0.0001 \\
\hline $21 \mathrm{C}$ & 0.1691 & 0.0371 & $57 \mathrm{C}$ & 0.0372 & 0.0384 \\
\hline $22 \mathrm{C}$ & -0.054 & -0.0028 & $58 \mathrm{C}$ & 0.1398 & -0.0092 \\
\hline $23 \mathrm{C}$ & 0.1973 & 0.0297 & $59 \mathrm{C}$ & 0.1844 & 0.0378 \\
\hline $24 \mathrm{H}$ & -0.0542 & 0.0004 & $60 \mathrm{C}$ & -0.0569 & -0.0028 \\
\hline $25 \mathrm{H}$ & 0.0686 & -0.0009 & $61 \mathrm{C}$ & 0.1974 & 0.0294 \\
\hline $26 \mathrm{H}$ & 0.0009 & 0.0003 & $62 \mathrm{H}$ & -0.0554 & 0.0004 \\
\hline $27 \mathrm{H}$ & 0 & 0.0001 & $63 \mathrm{H}$ & 0.0685 & -0.0009 \\
\hline $28 \mathrm{H}$ & 0.0304 & 0 & $64 \mathrm{H}$ & 0.001 & 0.0003 \\
\hline $29 \mathrm{H}$ & 0.0315 & 0 & $65 \mathrm{H}$ & 0.0001 & 0.0002 \\
\hline $30 \mathrm{H}$ & 0.0178 & 0.0001 & $66 \mathrm{H}$ & 0.0305 & 0 \\
\hline $31 \mathrm{H}$ & -0.0398 & 0.0004 & $67 \mathrm{H}$ & 0.0314 & -0.0001 \\
\hline $32 \mathrm{H}$ & -0.0489 & -0.0011 & $68 \mathrm{H}$ & 0.0177 & 0.0001 \\
\hline $33 \mathrm{H}$ & -0.0121 & 0.0002 & $69 \mathrm{H}$ & -0.0455 & 0.0004 \\
\hline $34 \mathrm{H}$ & 0.1528 & -0.0014 & $70 \mathrm{H}$ & -0.0559 & -0.0012 \\
\hline $35 \mathrm{H}$ & -0.0191 & 0.0001 & $71 \mathrm{H}$ & -0.0674 & -0.001 \\
\hline $36 \mathrm{H}$ & 0.0057 & 0.0023 & & & \\
\hline
\end{tabular}


Table S32. Calculated single-point energies of the different spin states of iron porphyrinogens at $0 \mathrm{~K}$.

\begin{tabular}{|c|c|c|c|c|c|}
\hline \multicolumn{2}{|c|}{$\left[\mathrm{LFe}^{\mathrm{II}}\right]^{2-}$} & \multicolumn{2}{|r|}{$\left[\mathrm{LFe}^{\mathrm{III}}\right]^{-}$} & \multicolumn{2}{|c|}{$\left[\mathrm{L}^{\Delta \Delta} \mathrm{Fe}^{\mathrm{II}}\left(\mathrm{NCCH}_{3}\right)\right]^{2+}$} \\
\hline$S$ & $\Delta U\left(\mathrm{~kJ} \mathrm{~mol}^{-1}\right)$ & $S$ & $\Delta U\left(\mathrm{~kJ} \mathrm{~mol}^{-1}\right)$ & $S$ & $\Delta U\left(\mathrm{~kJ} \mathrm{~mol}{ }^{-1}\right)$ \\
\hline 1 & 0 & $3 / 2$ & 0 & 2 & 0 \\
\hline 2 & 134 & $1 / 2$ & 51 & 1 & 20 \\
\hline 0 & 169 & $5 / 2$ & 165 & 0 & 89 \\
\hline
\end{tabular}

